\title{
A genomic view of estrogen actions in human breast cancer cells by expression profiling of the hormone-responsive transcriptome
}

\author{
Luigi Cicatiello', Claudio Scafoglio', Lucia Altucci ${ }^{1}$, Massimo Cancemi ${ }^{1}$, \\ Guido Natoli ${ }^{1}$, Angelo Facchiano ${ }^{2}$, Giovanni lazzetti ${ }^{3}$, Raffaele Calogero ${ }^{4}$, \\ Nicoletta Biglia ${ }^{6}$, Michele De Bortoli ${ }^{5,7}$, Christian Sfiligoi ${ }^{7}$, Piero Sismondi ${ }^{6,7}$, \\ Francesco Bresciani ${ }^{1}$ and Alessandro Weisz' \\ ${ }^{1}$ Dipartimento di Patologia generale, Seconda Università degli Studi di Napoli, Vico L. De Crecchio 7, 80138 Napoli, Italy \\ 2Istituto di Scienze dell'Alimentazione del Consiglio Nazionale delle Ricerche, Avellino, Italy \\ 3Dipartimento di Genetica, Biologia generale e molecolare, Università di Napoli 'Federico II', Napoli, Italy \\ ${ }^{4}$ Dipartimento di Scienze cliniche e biologiche, Università degli Studi di Torino, Torino, Italy \\ ${ }^{5}$ Dipartimento di Scienze oncologiche, Università degli Studi di Torino, Torino, Italy \\ ${ }^{6}$ Dipartimento di Discipline ostetriche e ginecologiche, Università degli Studi di Torino, Torino, Italy \\ ${ }^{7}$ Laboratorio di Ginecologia oncologica, Istituto per la Ricerca e la Cura del Cancro, Candiolo, Italy \\ (Requests for offprints should be addressed to A Weisz; Email: alessandro.weisz@unina2.it)
}

\begin{abstract}
Estrogen controls key cellular functions of responsive cells including the ability to survive, replicate, communicate and adapt to the extracellular milieu. Changes in the expression of 8400 genes were monitored here by cDNA microarray analysis during the first $32 \mathrm{~h}$ of human breast cancer (BC) ZR-75.1 cell stimulation with a mitogenic dose of $17 \beta$-estradiol, a timing which corresponds to completion of a full mitotic cycle in hormone-stimulated cells. Hierarchical clustering of 344 genes whose expression either increases or decreases significantly in response to estrogen reveals that the gene expression program activated by the hormone in these cells shows 8 main patterns of gene activation/inhibition. This newly identified estrogen-responsive transcriptome represents more than a simple cell cycle response, as only a few affected genes belong to the transcriptional program of the cell division cycle of eukaryotes, or showed a similar expression profile in other mitogen-stimulated human cells. Indeed, based on the functions assigned to the products of the genes they control, estrogen appears to affect several key features of BC cells, including their metabolic status, proliferation, survival, differentiation and resistance to stress and chemotherapy, as well as RNA and protein synthesis, maturation and turn-over rates. Interestingly, the estrogen-responsive transcriptome does not appear randomly interspersed in the genome. In chromosome 17, for example, a site particularly rich in genes activated by the hormone, physical association of co-regulated genes in clusters is evident in several instances, suggesting the likely existence of estrogen-responsive domains in the human genome.
\end{abstract}

Journal of Molecular Endocrinology (2004) 32, 719-775

\section{Introduction}

The identification of the gene expression patterns associated with cell responses to physiological stimuli, such as those represented by the female sex hormones estrogen and progesterone, may provide the framework necessary for interpreting the biological significance of specific gene expression patterns observed in pathologies. In the case of estrogen, this is particularly true also for the

The entire data set from these experiments, including the full list of genes analyzed and hormone regulated, the fluorescence measurement data and corresponding images can be found at the Gene Expression Group web site: (http://crisceb.unina2.it/geneexpression/), and will be deposited in the NCBI's Gene Expression Omnibus gene expression data repository (http://www.ncbi.nlm.nih.gov/geo/). 
identification of gene sets useful to monitor the hormone signaling status in the cell and, in the case of breast cancer (BC), to subtype tumor specimens according to this functional phenotype. Indeed, hormone-responsive breast tumors are currently identified primarily by the presence of estrogen receptor-alpha (ER- $\alpha)$ and the estrogenregulated progesterone receptors (PgRs). Characterization of the estrogen-responsive transcriptome would make it possible to go beyond individual genes or markers (in this case ER and PgR), allowing the analysis of hormone-related expression patterns across hundreds of genes (the estrogen-responsive transcriptome). This approach has the potential to lead to the identification of cancer-specific molecular 'signatures' characterizing previously undefined ER-positive tumor subtypes with distinctive biological and clinical behavior. Indeed, genome scale gene expression profiling is a promising new way to classify diseases in clinically relevant subtypes, according to multiple molecular parameters of the pathologic tissue. This can best be achieved when gene activity can be related to specific genetic or biological features of a given cell type (Alizadeh et al. 2000, Perou et al. 2000, Hedenfalk et al. 2001). Furthermore, identification of genes and whole genetic pathways responsive to estrogen can provide the necessary framework to understand the complex effects of these hormones and their receptors in target cells. Contrary to most other tumor promoters, estrogens do not act via 'classical' mitogenic pathways, but recognize intracellular receptors (ER- $\alpha$ and $-\beta$ ) which belong to the nuclear receptor family of transcription factors. In addition, direct and indirect effects of estrogen on extra-nuclear mitogenic signaling cascades have been proposed to contribute to the growth-promoting actions of these steroids (Moggs \& Orphanides 2001), pointing to the fact that estrogen's actions in target cells might involve both 'genomic' and 'non-genomic' signaling. An open question concerning the biology and pharmacology of ERs concerns the extent of integration between the genomic and extragenomic pathways, to permit, for example, promotion of cell proliferation by these steroids. A unifying way to clarify the issues listed above can be provided by new sets of estrogen target genes, each provided with information concerning its regulation by the hormone and used to identify hormone-regulated processes in target cells.

Hormone-responsive BC cell lines represent one of the best experimental models available to elucidate estrogen's actions in target tissues. Their systematic study has so far led to the discovery of several hormone-responsive pathways, which have then been exploited to improve clinical management of breast and other hormone-responsive cancers. These include, for example, identification of $\mathrm{PgR}$ as a downstream target of estrogen as well as an in depth investigation of the mechanism of action of the ERs, antiestrogens and selective estrogen receptor modulators (SERMs). Thus, it can be reasonably assumed that genome-scale gene expression analyses of hormone-responsive BC cell response to estrogen will lead to a better understanding of the hormonal influences in this cell type not only in vitro but also in vivo. Indeed, Bouras et al. (2002), by analyzing the response of MCF-7 cells to estrogen by gene expression profiling with DNA microarrays, identified the Stanniocalcin 2 gene as a hormone-responsive gene co-expressed with ER- $\alpha$ in BC lesions. Using a reverse approach, Finlin et al. (2001) found that expression of a novel gene, identified by microarray analysis in ER-positive breast tumors showing a slow tumor cell proliferation rate and a more favorable prognosis and named RERG (Ras-related, estrogen-regulated and growth-inhibitory), can be enhanced by estrogen in MCF-7 cells. Forced expression of this gene reduces the cell proliferation rate in vitro and inhibits their tumorigenic phenotype in nude mice (Finlin et al. 2001). Also, Inoue et al. (2002) and Soulez and Parker (2001) identified new estrogen target genes in BC cells which appear to be unaffected by antiestrogen treatment.

To provide a more detailed and comprehensive description of the gene expression patterns regulated by estrogen in $\mathrm{BC}$ cells, comparative cDNA microarray mRNA analysis was carried out before and after stimulation of hormone-responsive ZR-75.1 cells with a physiological dose of $17 \beta$-estradiol (E2). Gene expression changes were monitored in hormone starved cells at 11 consecutive times ranging from 1 to $32 \mathrm{~h}$ into hormonal stimulation, in order to analyze gene expression changes in the cells during a whole mitotic cycle (Gicatiello et al. 2000). Validation of the microarray results was accomplished 
systematically, by both experimental and in silico testing. The set of 344 hormone-responsive genes hereby identified depict a comprehensive genomewide view of the genetic programs regulated by estrogen in this hormone-responsive cell type.

\section{Materials and methods}

\section{Cell culture and RNA purification}

Human breast cancer ZR-75·1 and MCF-7 cells were propagated in DMEM medium supplemented with 5\% FBS and antibiotics $(100 \mathrm{U} / \mathrm{ml}$ penicillin, $100 \mu \mathrm{g} / \mathrm{ml}$ streptomycin and $250 \mathrm{ng} / \mathrm{ml}$ amphotericin-B). Before use, cells were plated at 20-40\% confluence and maintained for 4 days in steroid-free medium (phenol red-free DMEM medium, with 5\% charcoal-stripped FBS and antibiotics), before stimulation with $5 \times 10^{-8} \mathrm{M}$ E2. In the case of MCF-7 cells, hormone-deprived cultures were also maintained for $42 \mathrm{~h}$ in steroid-free medium containing $20 \mu \mathrm{M}$ simvastatin, to achieve effective $\mathrm{G}_{0}-\mathrm{G}_{1}$ synchronization of the cultures and optimal response of cell cycle genes to the hormone (Altucci et al. 1996, Bonapace et al. 1996). At the indicated times, total cellular RNA was extracted from control $(0,-\mathrm{E} 2)$ or E2stimulated (+E2) cultures as described earlier (Bonapace et al. 1996). Six culture plates (150 mm) were used for each time-point, and the experiment was repeated three times within two weeks. Poly $(\mathrm{A})^{+}$RNA was isolated from pools of $2 \mathrm{mg}$ total RNA, by batch-wise magnetic separation with Dynabeads (Oligo $(\mathrm{dT})_{25}$; Dynal, Oslo, Norway) according to the manufacturer's instructions, resuspended in diethyl pyrocarbonate-treated water, quantitated and diluted to a final concentration of $50 \mathrm{ng} / \mu \mathrm{l}$. Before further use, total and poly $(\mathrm{A})^{+}$RNAs were tested by agarose gel electrophoresis. The response to estrogen of the cell cultures to undergo microarray testing was controlled by cytofluorimetric cell cycle kinetics analysis, performed as described earlier (Bonapace et al. 1996), as well as by analysis of cell cycle and estrogen-responsive gene mRNAs (cyclins, pS2 and c-fos) by Northern blotting, as described earlier (Altucci et al. 1996 and data not shown), or by multi-probe RNase protection assays (RPAs).

\section{cDNA microarray probing}

cDNA microarray mRNA analysis was carried out with UniGEM Human $\mathrm{V} \mathrm{2 \cdot 0}$ and Human
UniGene 1 (for ZR-75·1 cell mRNA analyses) and UniGEM Human V (for MCF-7 cell mRNA analyses) glass arrays (Incyte Genomics, St Louis, MO, USA) according to standard procedures (Schena et al. 1995, Iyer et al. 1999). Two hundred nanograms of each poly $(\mathrm{A})^{+}$RNA derived from estrogen-stimulated (+E2) cells were used to generate carbocyanine 5 (Cy5)-labeled cDNAs, that were mixed with common reference probes consisting of carbocyanine 3 (Cy3)-labeled cDNAs prepared from an equal amount of $\operatorname{poly}(\mathrm{A})^{+} \mathrm{RNAs}$ purified from quiescent, hormone-deprived cultures ( $-\mathrm{E} 2$ ), for competitive hybridization to a cDNA microarray grid containing 9182 human sequences of known genes and expressed sequence tags (representing 8372 unique gene/ESTs clusters), spotted in single, and 192 internal controls (for reverse transcription, absolute sensitivity and differential expression QGs: human UniGEM V $2 \cdot 0$ screening services; Incyte Genomics). cDNA synthesis, labeling and hybridization were generally repeated three times for each time-point (in 3 cases four times), in separate experiments. For at least one sample/set (time-point), the fluorescent labeling order was interchanged (control RNAs were labeled with Cy5 and sample RNAs with Cy3).

\section{Statistical analysis of the microarray data}

Data considered for analysis were, for each element in the array, absolute Cy3 or Cy5 fluorescence reading, signal vs background ratio $(\mathrm{S} / \mathrm{B})$ and 'balanced differential expression' value. This was calculated as the ratio between the fluorescence signal in the sample channel (+E2 probe) vs that in the reference channel ( $-\mathrm{E} 2$ probe), after balancing (normalization) for differences in sample labeling efficiency and for laser performance which was based on the average hybridization signal of the whole array and on a set of housekeeping genes and other control elements present in multiple copies at different locations in the array grid. The data relative to elements of the array which did not pass the Incyte PCR quality controls, showing multiple bands or no amplification of the spotted DNA samples, as well as arrays that did not pass the Incyte microarray hybridization QCs, were discarded.

Identification of informative (expressed reproducibly to detectable levels) and hormone-regulated genes was carried out, in the case of the kinetics 
measurements, according to three independent selection procedures, applied in parallel by different operators to help pinpoint possible methodological biases. With the first, a normalization step procedure was applied to correct for the possible differences in cDNA labeling, hybridization or detection efficiency which are observed during multiple analyses of the same mRNA sample by this technology, as described by Dudoit et al. (2001). In each array, all data showing a $\mathrm{Cy} 3+\mathrm{Cy} 5$ value lower than 500 (equal to $1 / 5$ of the average Cy3+Cy5 value in all arrays) and a signal over background value (S/B) lower than 2 were discarded. This was necessary due to the lack of linear correlation between S/B vs Cy3+Cy5 observed for the vast majority of the analyzed data. In this way, 6080 genes were selected as informative, as they gave rise to reproducible hybridization signals. Subsequently, all the data where the ratio between the test and reference sample values diverged more than $15 \%$ within replicates were also discarded. For the remaining coherent replicates, the ratios between experimental time-points and their reference sample were calculated and averaged. All data showing a differential expression of at least $\pm 1 \cdot 5$ were first selected with this approach, as validation studies for the same microarray platform used for this study indicate that detection limits for reliable differential expression measurements are $\pm 1 \cdot 4$-fold (Yue et al. 2001).

According to a second selection scheme, each array was first statistically evaluated for its performance. The fluorescence values from the -E2 sample channel of each element were compared between all arrays, and used for calculation of an average (mean) fluorescence value and the corresponding standard deviation (S.D.). The absolute deviation of each element's fluorescence value $(\mathrm{F})$ from the corresponding mean (Fm) was then computed relative to the S.D., e.g. $\mathrm{abs}(\mathrm{F}-\mathrm{Fm}) / \mathrm{s}$.D. The result represents the difference between the signal detected by each given element in the array, and the corresponding mean was used as the discriminant to discard unreliable data points, which were found to include all elements that did not pass Incyte QCs or showed a low S/B ratio. All data showing a Cy3+Cy5 fluorescence reading lower than $1 / 5$ of the average $\mathrm{Cy} 3+\mathrm{Cy} 5$ value in each array were discarded. The remaining data sets were then used to identify differentially expressed genes, which were selected when their balanced differential expression value showed consistent variations in at least three consecutive time-points. Genes showing an average balanced differential expression of at least \pm 1.5 in at least two of three consecutive time-points were then selected as 'positive'.

The data relative to microarrays where the crude fluorescent values relative to the reference sample channel showed the highest S.D. (accounting for about one third of the total) were excluded from the third independent selection approach. All data were merged in this case in a single MS Excel file and the average balanced differential expression was calculated for each gene within 'time windows' including arrays covering three consecutive experimental time-points. Analysis was carried out sequentially over consecutive time windows overlapping one another by one time-point (e.g. window $A: 1 h+2 h+4 h$; window $B: 4 h+6 h+8 h$; etc.). In each case, genes showing an average balanced differential expression of at least $\pm 1 \cdot 4$ ( $>2 \times$ the mean balanced differential expression's S.D.)/time window were marked as positive. Once this section was completed, all results were merged in a single file, and genes scored positive when showing gradual and consistent changes over time, above the statistically set threshold of \pm 1.5 in at least two time-points of the curve.

The results of the three selection procedures described above were then compared, and 372 genes, common to all three data sets, were considered positive and finally selected. This procedure led us to discard 120 genes that were not selected by at least one of the procedures described above. These genes were all manually re-controlled and found to show inconsistencies of differential expression value among replicates, or to fail the criterion of consistent variation over multiple consecutive time-points. Furthermore, 27 of the 372 positive genes were present in two or more copies in the array, generally under a different gene name or GenBank Accession number, and were identified as belonging to the same UniGene cluster by automatic database searches and BLAST sequence alignments. Interestingly, balanced differential expression data were found to be consistent between replicates, providing a further demonstration in support of the performance of the procedures adopted for data analysis and of the selection criteria set to mark differentially expressed 
genes. In conclusion, 345 genes were identified in this way as estrogen regulated, one of which was later discarded during experimental validation of the microarray results (see below).

A statistical analysis of the gene expression changes defined in this way was further carried out by the method proposed by Tusher et al. (2001; significance analysis of microarrays SAM), which provides a way to determine the significance of the gene expression changes detected by cDNA microarrays. SAM identifies genes showing statistically significant changes in expression between mRNA samples by assimilating a set of gene-specific $t$-tests and assigning a score to each gene on the basis of its changes in expression relative to the standard deviation for repeated measurements. Furthermore, it uses permutations of replicate measurements to estimate the 'false discovery rate' (FDR), e.g. the percentage of genes identified by chance. This method was applied to the sets of data generated as described above, by implementing the relative software (http://www.stat-class.stanford.edu/ SAM/SAMServlet) following the recommendations provided by the A.s (Tusher et al. 2001). To enhance the robustness of the analysis, we selected to apply the SAM analysis also to samples grouped in 8 time-windows, each encompassing $4 \mathrm{~h}$ and overlapping with the neighboring windows by one time-point (e.g. $1 \sim 4 \mathrm{~h}, 4 \sim 8 \mathrm{~h}$, $8 \sim 12 \mathrm{~h}$, etc.). We found in this way that the number of significant genes decreased only slightly, when compared with single-point analyses, while the FDR value decreased significantly (to 0.2 to 0.5 in most cases). The data relative to 312 of the 344 genes selected initially (91\%) were significant, according to this additional statistical analysis, in at least 6 of the 8 data sets considered. To account for the longitudinal nature of the experiment (estrogen stimulation of the cells was carried out for $32 \mathrm{~h}$ and measurements were performed at 11 time-points, grouped in 8 consecutive time-windows), the SAM differential expression data (fold-change) were compared with the raw differential expression data (average of multiple microarray measurements, as described above). This was performed by calculating, in each case, the corresponding Pearson's correlation coefficient. All genes showed very similar differential expression profiles in the two data sets resulting from the two distinct analytical proce- dures, providing a robust confirmation of the gene expression changes reported.

Differentially expressed genes were clustered according to their expression profiles by GeneCluster (Tamayo et al. 1999). The expression profiles obtained with these two approaches were compared and each gene cluster was visually inspected, to give rise to the final set of coherent clusters, with the aid of Cluster and TreeView tools (Eisen et al. 1998).

The data sets relative to these experiments, including the list of all genes analyzed and, separately, of those hormone regulated, the fluorescence measurements data and the corresponding scanning images can be viewed at the Gene Expression Group web site (http:// crisceb.unina2.it/geneexpression/) and will be made available also through the NCBI's Gene Expression Omnibus gene expression data repository (http://www.ncbi.nlm.nih.gov/geo/).

\section{Real-time rtPCR}

Real time, quantitative rtPCR analyses (Heid et al. 1994) were performed with an ABI Prism 7700 Sequence Detector and reagents provided by the same manufacturer (PE Applied Biosystems, Milan, Italy). cDNA sequences present in the arrays were obtained from Incyte Genomics and checked by comparison with the corresponding GenBank sequence files. Specific primers and probes for the mRNAs to be analyzed were designed using the dedicated software Primer Express. Amplicons of 64-101 bps were selected for testing. Melting temperatures of the primers were $59-61{ }^{\circ} \mathrm{C}$ and melting temperatures of the probes were $69-71{ }^{\circ} \mathrm{C}$. rtPGR reactions were performed using amounts of cDNA corresponding to $0 \cdot 025-0 \cdot 125 \mu \mathrm{g}$ DNasetreated total RNA, as recommended by the manufacturer. Each $25 \mu \mathrm{l}$ of reaction mix contained $2 \times$ Universal Master Mix, $100 \mathrm{nM}$ TaqMan probe, $20 \times$ PDAR 18S (pre-developed TaqMan assay reagent), $300 \mathrm{nM}$ of each primer (except for AF022224, L16785, AF016050, U75285, D38553, AI568937 and AI741331: 300/600 nM, AA865269: 600/300 nM and U22376: $600 / 600 \mathrm{nM}$ ) (all these reagents were from PE Biosystems, Milan, Italy). After 2 min at $50{ }^{\circ} \mathrm{C}$, to allow AmpErase uracil-N-glycosilase (UNG) to destroy potential contaminating PCR products, and $10 \mathrm{~min}$ at $95^{\circ} \mathrm{C}$ to denature UNG 
and activate Taq polymerase, amplification was then carried out for 40 cycles, each of $15 \mathrm{~s}$ at $95{ }^{\circ} \mathrm{C}$ and $1 \mathrm{~min}$ at $60^{\circ} \mathrm{C}$. All amplified samples were also resolved in a $2.5 \%$ agarose gel to verify the expected amplicon size. A dissociation curve was generated at the end of the PCR cycle to verify that a single product was amplified.

The sequences of the PCR primer pairs and of the fluorogenic probe $\left(5^{\prime}\right.$ to $\left.3^{\prime}\right)$, respectively, that were used for each gene are as follows: AA194161: TGAGGAGCTCATGGTAGCCTGGGTTG, GG TGACAGATTCACGAAAGGTGA, and 6FAMAGACACGGTGCTCGAGAGTAGGT-TAMRA; AA865269: CGGGCTTTCGTGGGTGGGGG, AGGAAAACAGGGGAAAGGTCTTG, and 6FAM-GTGGAGAGAGCGGCACACG-TAMRA; AF016050: TCTGGGTCGCACGTCGGCACA, GAACTCTGCCGACTGCATGAC, and 6FAMTGCTGTGGCTTCTGGTAGGG-TAMRA; AF022224: TTTAGCTGATTTGTTGAGGGGTGGTGGG, GACGAGCTCTGGCGTGTCTG, and 6FAM-AAATTGGGAAATGGCGAGTTGTAMRA; AF030424: CGAGACAAAACGCGG CGACGTGTA, TTGGGACGGTAGGGTACAT GA, and 6FAM-ATGGAGTCAAAATCAGCA TCTGACT-TAMRA; AF0501 10: TAACGACGAGACGTGCCGAATGAAAGC, AGTGTGATT GGTGATAGAGCTGATG, and 6FAM-AAG AATTGTTGTGATAGTTGAGGATGGT-TAM RA; AF068235: AAGGCAGGGGTTCGGGG GAA, GGCGTATCTCGCTCAGAAGGG, and 6FAM-TAGTAGGCGCTCGTTTCGGG-TAM RA; AF109161: CCCTGCTGGAGGCGACGAGATG, CGACTACATGCGGGATTTGG, and 6FAM-TCGGAAGTGCTGGTTTGTCG-TAMRA; AI310309: GTTGGAGAGGGGATTTGGATTG GGTT, AGACGTGTTTTTCGTTGCGACA, and 6FAM-TCAGGGCAAGAAACGTCTTCT AAA-TAMRA; AI34807 1: CATCGGCTGACAT GGGCACGG, TTGCGGGAAGGGAGGTAAG, and 6FAM-GGGTAGATCGCTCGAAAGCAGTAMRA; AI568937: CGACGGATCGGAGGTT TTGGCA, TAGCAGGAATGCTAGAGCGATATGAT, and 6FAM-GTAACTACCATGGCT GCGGAAGTTT-TAMRA; AI741331: ACTCACGCAAGGCGCGCAGGGA, CTGGGCAAGGA GGAGCGTTAG, and 6FAM-TGCTCGCAA CGCATTCACTACA-TAMRA; D38553: CGCG CTGAAGGTGGGTGGTGA, TGGATGATAGT GTGGGTGCGA, and 6FAM-CTGCGCTCGCG TGTCGTAGAT-TAMRA; L16785: TGGCGAT-
GAAGTTCGTCGGGGG，CGGTTCGAGCAG AAGGGATT, and 6FAM-AATGGTCGGT GTTTCAGGTCAA-TAMRA; M31732: TGTTGCTAGAGGGGGGTGGCGAC, AGTGAACA CGGAGTGCGAAGAA, and 6FAM-CGCT CTTAATGTCGACTGCGTC-TAMRA; U22376: ACAGCTTCAGGAAAGAGAGGGGAATGG, AA CATCACACAGGCAAAGCGC, and 6FAMCATAGGTTTGTTGACGCTTGGG-TAMRA; U22970: GGTGCGAAACACGGTGGGAATCG, TCATGATATTGACATCTGGGGAC, and 6FAM-CTGCGCGAACGGGTGG-TAMRA; U44427: TGGAGGCGGAGGGACAAGGTTTG, TCAGGTGGAAGTCAGGCACG, and 6FAMTGGAACGGTTCAGTCTCGAAC-TAMRA; U75285: GTTTCGACGCTCATTGCTTCTTAA ACAGGTG, AAAAGATTTGAGTTGCAAAGA CACTTAG, and 6FAM-GGATATAGAGAGG TATGCGTTCACAA-TAMRA; X55150: CGCA CGAGGTAAGGACATTTCGAGGGTT, ATTC CTGATGGCGAAGCTTG, and 6FAM-TCGCA GGACGAGGCCCTAT-TAMRA.

Threshold cycle, Ct, which correlates inversely with the target cDNA levels, was measured as the cycle number at which the reporter fluorescent emission increased above a threshold level. To obtain a quantitation of target cDNA, as relative to the zero point, $\mathrm{Ct}$ of each sample was substracted from the $\mathrm{Ct}_{0}$, and each of them was corrected for the Ct of the 18S ribosomal RNA, used as an internal reference and co-amplified with target, as described above. The relative quantity of the template $(Q)$ was calculated as: $Q=2-(\mathrm{Ct}-\mathrm{Ctr})-(\mathrm{Ct} 0-\mathrm{CtOr})$. Two independent RNA preparations were analyzed, all samples were run in triplicate and the mean and standard deviation calculated as the square root of: $\operatorname{Var}(\mathrm{Q}){ }_{\mathrm{e}}-2 \mu \ln 2+2 \sigma^{2}(\ln 2)^{2}{ }_{[\mathrm{e}} \sigma^{2}(\ln 2)^{2}{ }_{-1]}$ where $\mu=$ the mean of $(\mathrm{Ct}-\mathrm{CTr})-\left(\mathrm{Ct}_{0}-\mathrm{Ct}_{\mathrm{Or}}\right)$ and $\sigma$ its standard deviation. To compare measurements from replicate determinations, data from one experimental series were normalized by correcting for the mean ratio between series, and the data were finally averaged.

\section{Multi-probe RNase protection assays (RPAs)}

Human Apoptosis 2c and 5, and Human Cyclin 1 template sets (Pharmingen, San Diego, CA, USA) were used according to the manufacturer's instructions. Probes were labeled with $\alpha-{ }^{32} \mathrm{P}$ uridine 
triphosphate (NEN Italia, Milan, Italy) and the ribonuclease (RNase) protection assay was performed on total RNA $(4 \mu \mathrm{g})$ with 6 to $8 \times 10^{5}$ d.p.m. of labeled probe mix. After RNase digestion, the protected probes were resolved on urea- $5 \%$ polyacrylamide-bis-acrylamide standard sequencing gels. Dried gels were placed on Hyperfilm-MP autoradiographic films (Amersham Italia) in a cassette with an intensifying screen. Exposure time was between 12 and $48 \mathrm{~h}$, depending upon the intensity of the individual signals to be visualized. Band quantitation was carried out by densitometric scanning of the autoradiograms.

\section{In silico data analyses}

Each gene under study was assigned to its actual UniGene cluster (http://www.ncbi.nlm.nih.gov/ UniGene/) and databases were searched by UniGene cluster and GenBank Accession numbers. A functional category was assigned to each gene according to Gene Ontology (http://www. godatabase.org/cgi-bin/go.cgi), from LocusLink (http://www.ncbi.nlm.nih.gov/LocusLink/), GeneCards (http://bioinfo.weizmann.ac.il/cards/) or Genatlas (http://bisance.citi2.fr/GENATLAS/) databases of human genes. SAGE libraries representing mRNA tags expressed in estrogen deprived or stimulated ZR-75·1 (Seth et al. 2002) and MGF-7 (Charpentier et al. 2000) cells were queried with Xprofiler and Tag-to-Gene (http:// www.ncbi.nlm.nih.gov/SAGE/). Alignment of the estrogen-responsive transcriptome identified here in ZR-75.1 cells with those identified previously in MCF-7 cells (Finlin et al. 2001, Bouras et al. 2002, Inoue et al. 2002, Lobenhofer et al. 2002, Hodges et al. 2003) was carried out by matching datasets by UniGene cluster number. Comparisons between estrogen-regulated genes in $\mathrm{BC}$ cells and serumregulated genes in human fibroblasts (Iyer et al. 1999) or genes periodically expressed during the cell cycle in human cells (Whitfield et al. 2002) were carried out by matching datasets by UniGene cluster number. Where necessary, they were also performed according to gene description or GenBank Accession number. A cytogenetic location was assigned to each gene according to LocusLink, GeneCards and/or Ensembl (http://www.ensembl.org/Homo_sapiens/) databases. Estrogen-responsive chromosomal domains were investigated on the UG Santa Cruz 'Golden
Path' human genome browser (http://genome. ucsc.edu/cgi-bin/hgGateway?org=Human).

\section{Results and discussion}

\section{cDNA microarray analysis of gene expression changes in estrogen-stimulated ZR-75.1 cells}

ZR-75.1 cells express ER- $\alpha$ and show well characterized responses to estrogen and antiestrogen both in vitro and in vivo (Cicatiello et al. 2000, Couillard et al. 2000), representing a reliable cellular model to study estrogen actions in target cells. Hormone deprivation of ZR-75.1 cells induces $\mathrm{G}_{1}$ arrest, as shown in Fig. $1 a$ by the low number of $\mathrm{S}$ and $\mathrm{G}_{2} / \mathrm{M}$ cells in the culture $(10 \cdot 4$ and $7 \cdot 7 \%$ respectively), that results in growth arrest (Cicatiello et al. 2000 and data not shown). This quiescence status can be readily overrun by cell exposure to physiological concentrations of estrogen, that is followed by resumption of cell cycle progression $(52.7 \%$ cells in $\mathrm{S}$ phase after $28 \mathrm{~h}$ of stimulation; Fig. 1a), and activation of the cyclins-CDKs (cyclin dependent kinases) cell cycle control pathway. This last effect was highlighted by the timed accumulation of $\mathrm{G}_{1}$ cyclin D1 and D3 mRNAs during the first 1 to $12 \mathrm{~h}$ of stimulation, and of the $\mathrm{S}$ and $\mathrm{G}_{2} / \mathrm{M}$ cyclin $\mathrm{A}$ and $\mathrm{B}$ mRNAs after 16 to $28 \mathrm{~h}$ (Fig. 1b,c).

Gene expression monitoring was carried out in hormone starved, quiescent ZR-75·1 cells before and at 11 time-points after stimulation with a mitogenic dose of $\mathrm{E} 2$ by cDNA microarray analysis. Cells were maintained under the same conditions used for the tests reported in Fig. 1. This experimental setting was selected to provide a comprehensive kinetic assessment of the gene responses to the hormone during a mitotic cycle (see Fig. 1b,c). The microarray platform used for this study included 9182 cDNA elements, representing 8372 randomly selected unique gene/ESTs clusters. cDNA was synthesized from mRNA extracted from cells stimulated with the hormone for the indicated time, labeled with the fluorescent Cy5 dye, mixed with an equal amount of a common reference target consisting of Cy3-labeled cDNA extracted from hormone deprived, quiescent cultures (time 0) and hybridized to a glass array as described in Materials and methods. Three to four independent hybridization reactions were carried out for each sample pair by independent cDNA synthesis, labeling and hybridization to different 
(a)
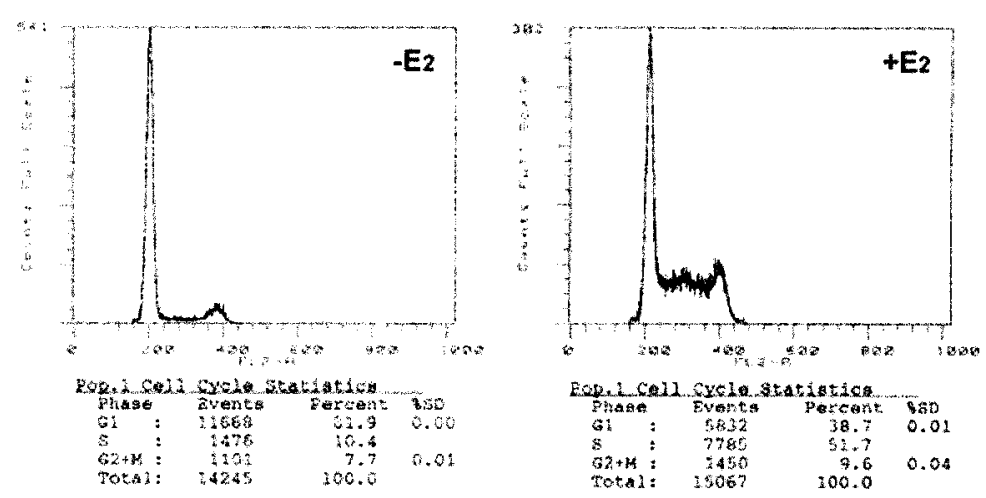

(b)

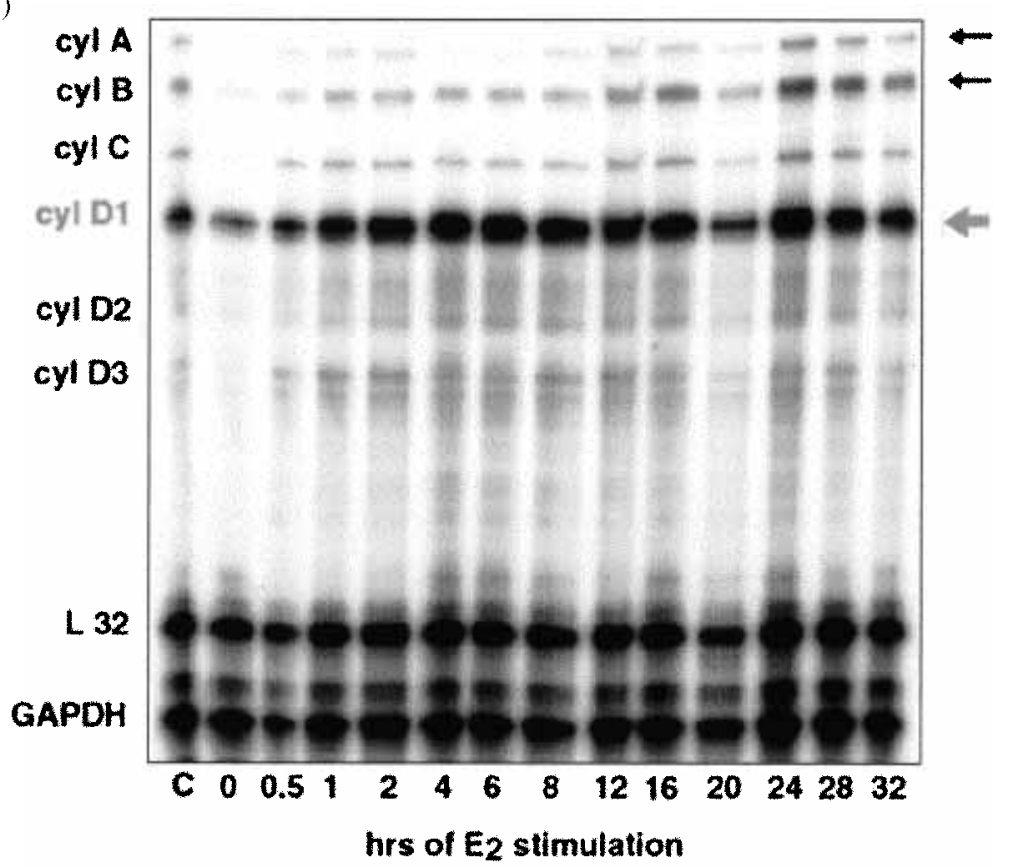

(c)

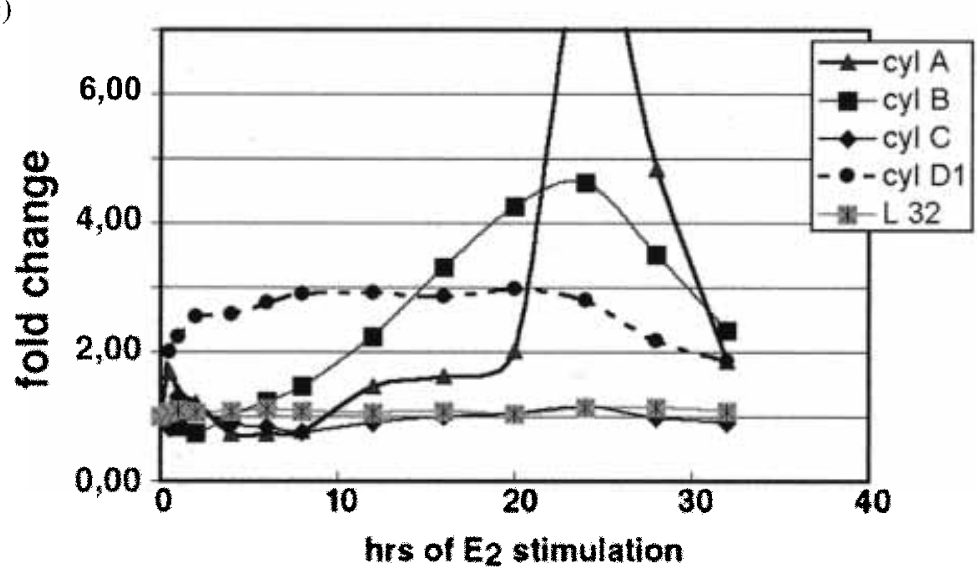


microarrays. Furthermore, in at least one of these replicates the fluorescent labels were exchanged between the two cDNAs analyzed in parallel, to provide a further control. Six thousand and eighty gene transcripts were clearly detectable under the experimental conditions employed, and were further analyzed to identify estrogen target genes of this cell line. Based on statistical evaluation of the results, a gene scored positive (hormone regulated) when it showed consistent changes in differential expression (+E2/ - E2) over multiple, sequential time-points and reached a threshold value of $\pm 1 \cdot 5$ within a time-window comprising two to three sequential measurements. This cutoff value was selected according to statistical parameters (see Materials and methods). In this way, 344 genes showing significant changes in activity in estrogen treated vs untreated cells were identified (Table 1).

As a first step to read the information on estrogen signaling and actions provided by the gene expression changes measured here, we used hierarchical clustering to organize the estrogenregulated genes according to similarities in their respective inhibition or activation kinetics. The rationale for this analysis resides in the consideration that the function of a gene and its expression pattern are tightly connected, and that genes linked by coherent expression profiles are likely to respond in a comparable fashion and according to similar mechanisms to information concerning the cell environment, state or identity. Indeed, all available information on gene regulation in higher eukaryotes suggests that common expression patterns underscore shared functions and/or regulation. The estrogen-responsive genes were found to fall within eight clusters (Fig. 2a), which summarize the main kinetic patterns of gene expression changes detectable in estrogen-stimulated BC cells. The first three clusters (1 to 3) group all down-regulated genes, the following three ( 4 to 6 ) comprise genes whose transient activation patterns relate kinetically to cell cycle phasing and the last two (7 and 8) include genes showing persistent activation by the hormone for up to $32 \mathrm{~h}$. Interestingly, the main determinant for clustering of genes showing progressive changes in expression profiles (clusters 1-3 and 7-8) was the onset of their response to the hormone. The difference between the patterns of inhibition shown by the genes in clusters 1, 2 and 3, for example, is represented by the time-point where a progressive decrease in mRNA expression can first be detected ( 1 to $4 \mathrm{~h}$ for cluster 1 genes, 6 to $8 \mathrm{~h}$ for those of cluster 2 and $\geq 12 \mathrm{~h}$ for cluster 3 ). Similarly, clusters 7 and 8 are distinguishable from each other by the fact that mRNA expression levels start to increase significantly before (cluster 7) or after (cluster 8) $12 \mathrm{~h}$ stimulation. It is worth mentioning that the time clusters identified here correspond quite well with those recently described by Frasor et al. (2003) in estrogen-stimulated MCF-7 cells.

Figure 2b reports a graphical display of the results obtained and, in addition, a further subclustering of the genes belonging to each cluster, indicated by the correlation tree (Eisen et al. 1998) reported to the left, which points to higher similarities between expression profiles within subgroups of genes. This highlights how defined groups of genes, within each of the 8 kinetic clusters identified, are characterized by slightly different expression profiles in hormone-stimulated cells. The definition associated with each gene symbol, and more information concerning each of the 344 estrogen-responsive genes depicted in this Figure are reported in Table 1.

\section{Microarray data validation}

The differential expression threshold selected to identify estrogen-regulated genes $( \pm 1 \cdot 5$-fold

Figure 1 Mitogenic responses of ZR-75.1 cells to estrogen stimulation. (a) Cytofluorimetric cell cycle analysis of estrogen-deprived cell cultures before (-E2) and $28 \mathrm{~h}$ after stimulation with $5 \times 10^{-8} \mathrm{M} 17 \beta$-estradiol (+E2). (b) Cyclin mRNA expression was monitored by RPA (RNase protection assay) in total RNA extracted from cells in log-phase growth $(\mathrm{C})$ or following estrogen deprivation, before $(0)$ and at the indicated time-points after stimulation with $5 \times 10^{-8} \mathrm{M} 17 \beta$-estradiol (E2). Cyclins D1, D2 and D3 (cyl D1, cyl D2 and cyl D3) are G1-phase markers, whereas cyclins A and B (cyl A and cyl B) indicate S/G2-phase progression. L32 and GAPDH are cell cycle- and estrogen-unresponsive mRNAs, used as internal controls in each test. Arrows mark regulated genes; the gray arrow indicates that CCND1 (cyl D1) mRNA also showed comparable changes by cDNA microarray analysis. (c) Graphic representation of data from mRNA expression monitoring by RPA. Average of results from multiple, independent measurements are reported only for regulated mRNAs and L32 control, following normalization with respect to GAPDH mRNA levels in each lane. 


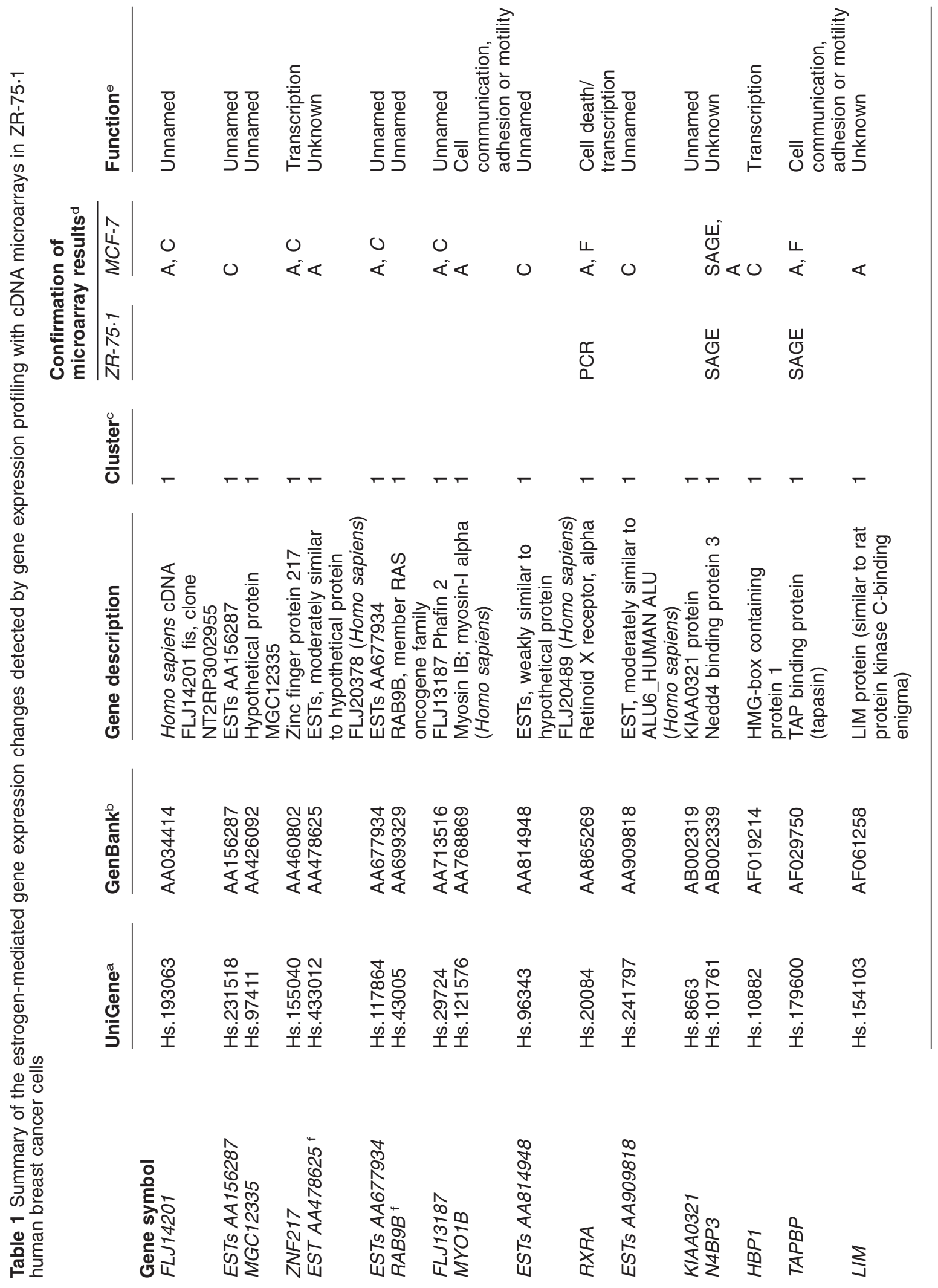



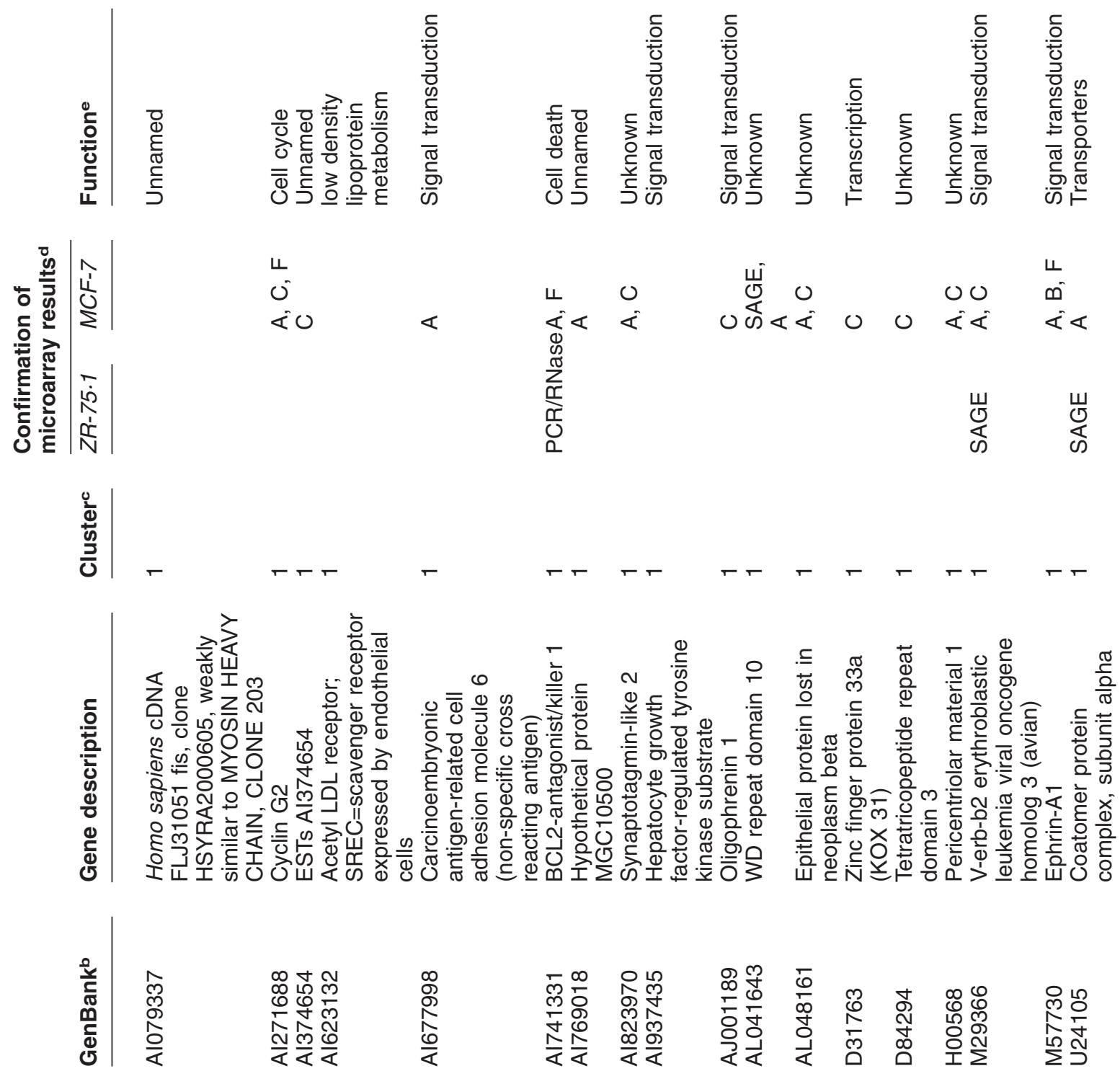

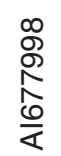

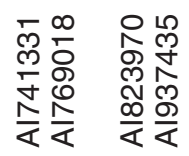

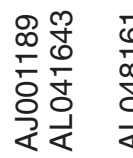

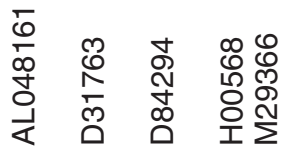

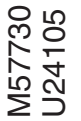

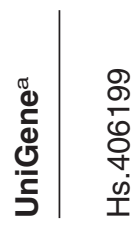

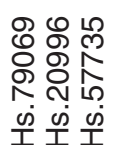

$\infty$
$\stackrel{\infty}{+}$
$\stackrel{\infty}{N}$
$\stackrel{\infty}{I}$

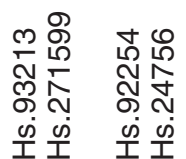

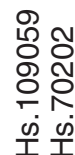

$\begin{array}{ll}0 & N \\ 0 & 0 \\ 0 & 0 \\ 0 & 0 \\ \text { i } & \frac{\infty}{1}\end{array}$

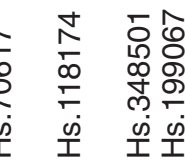

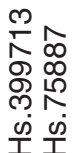

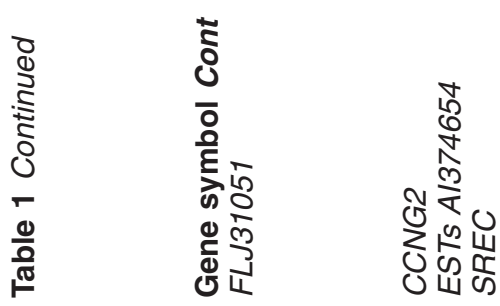

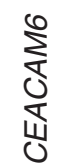

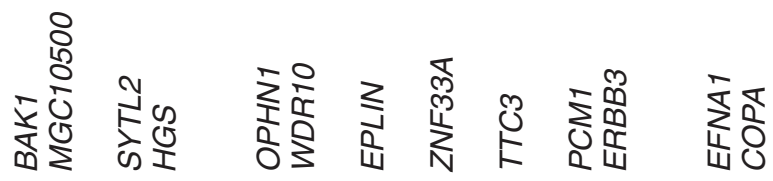



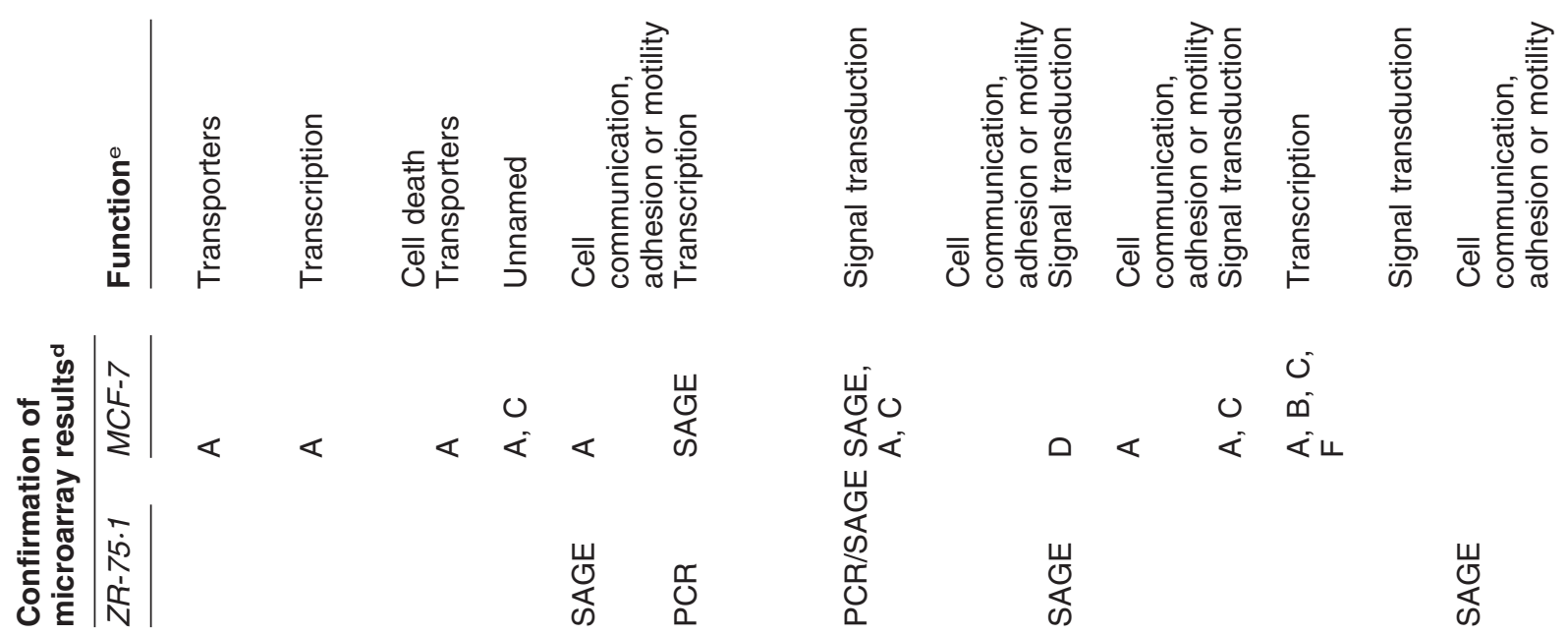

๘
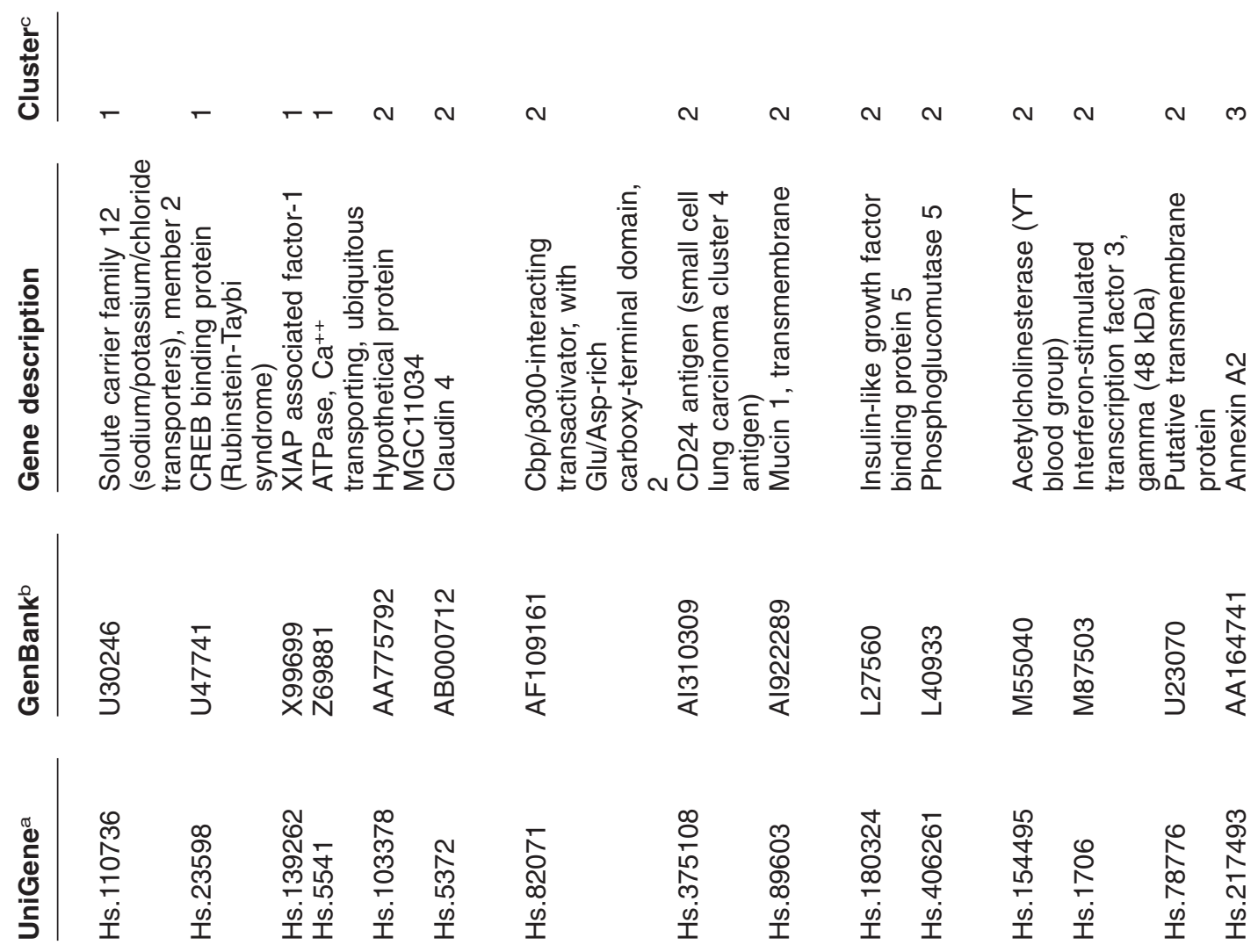

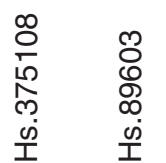

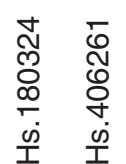

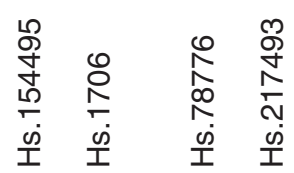

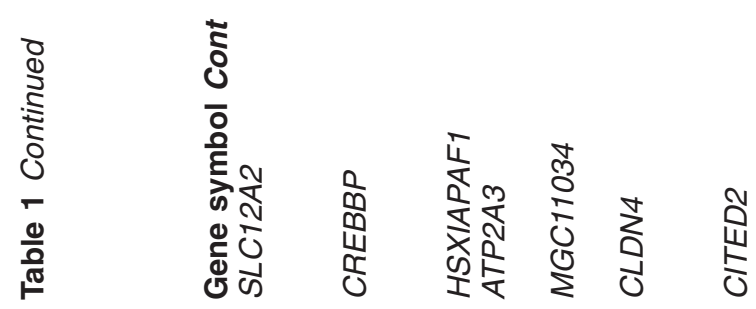

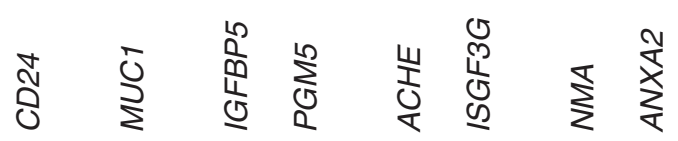



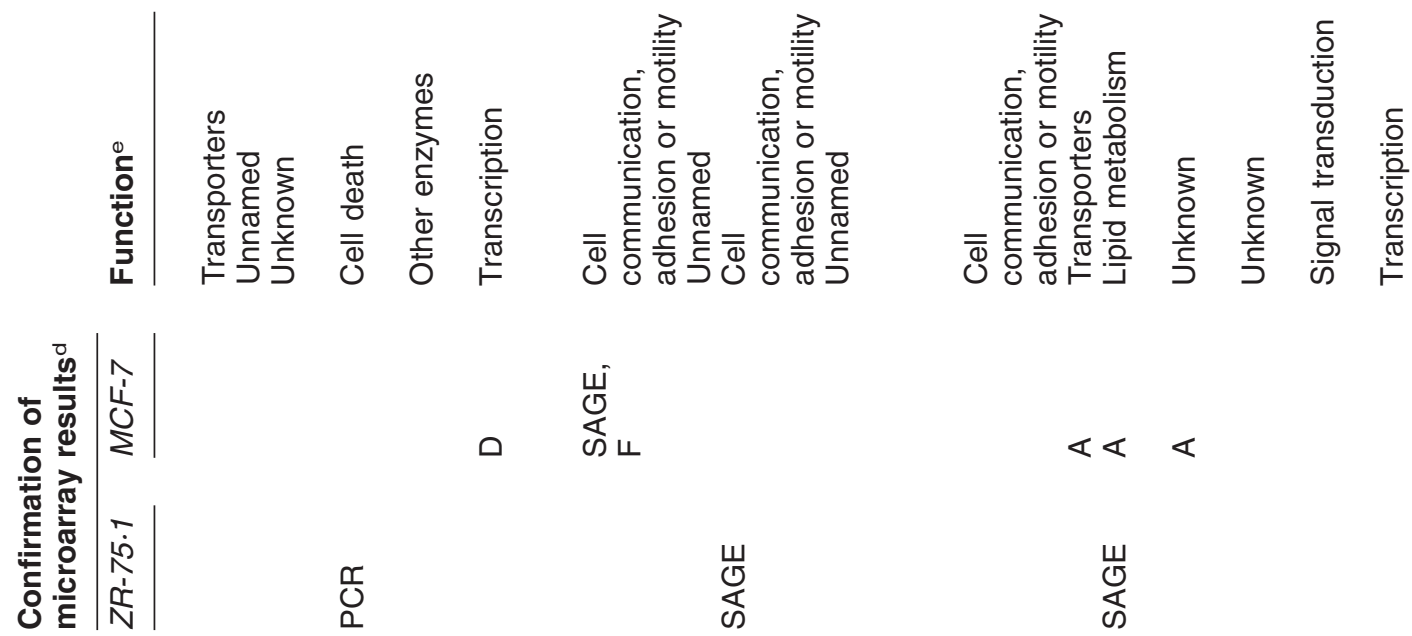

岕

凹

$\frac{0}{\frac{1}{2}}$

๓ ๓

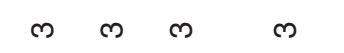

๓

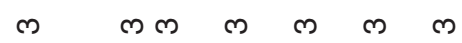
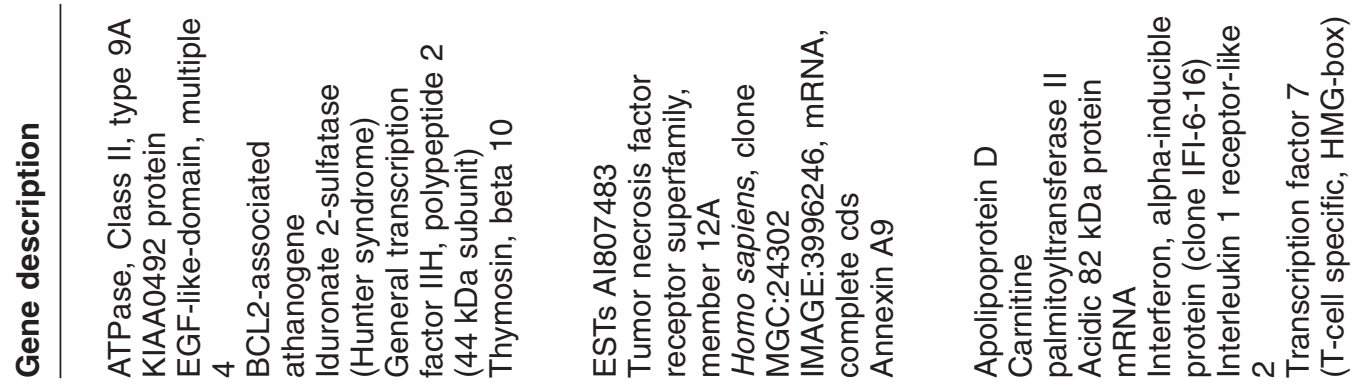

章

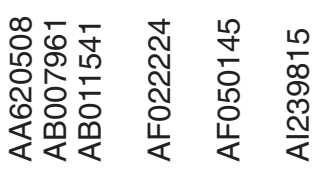

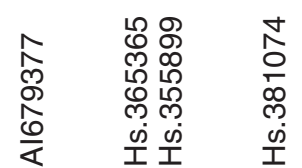

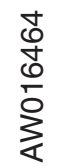

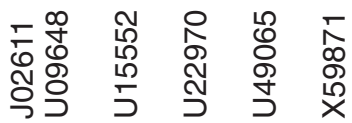

|

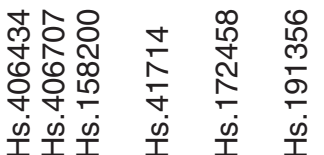

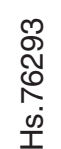

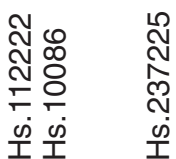

๙্ণ

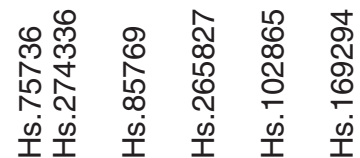

$\varangle \varangle \quad \varangle$ 

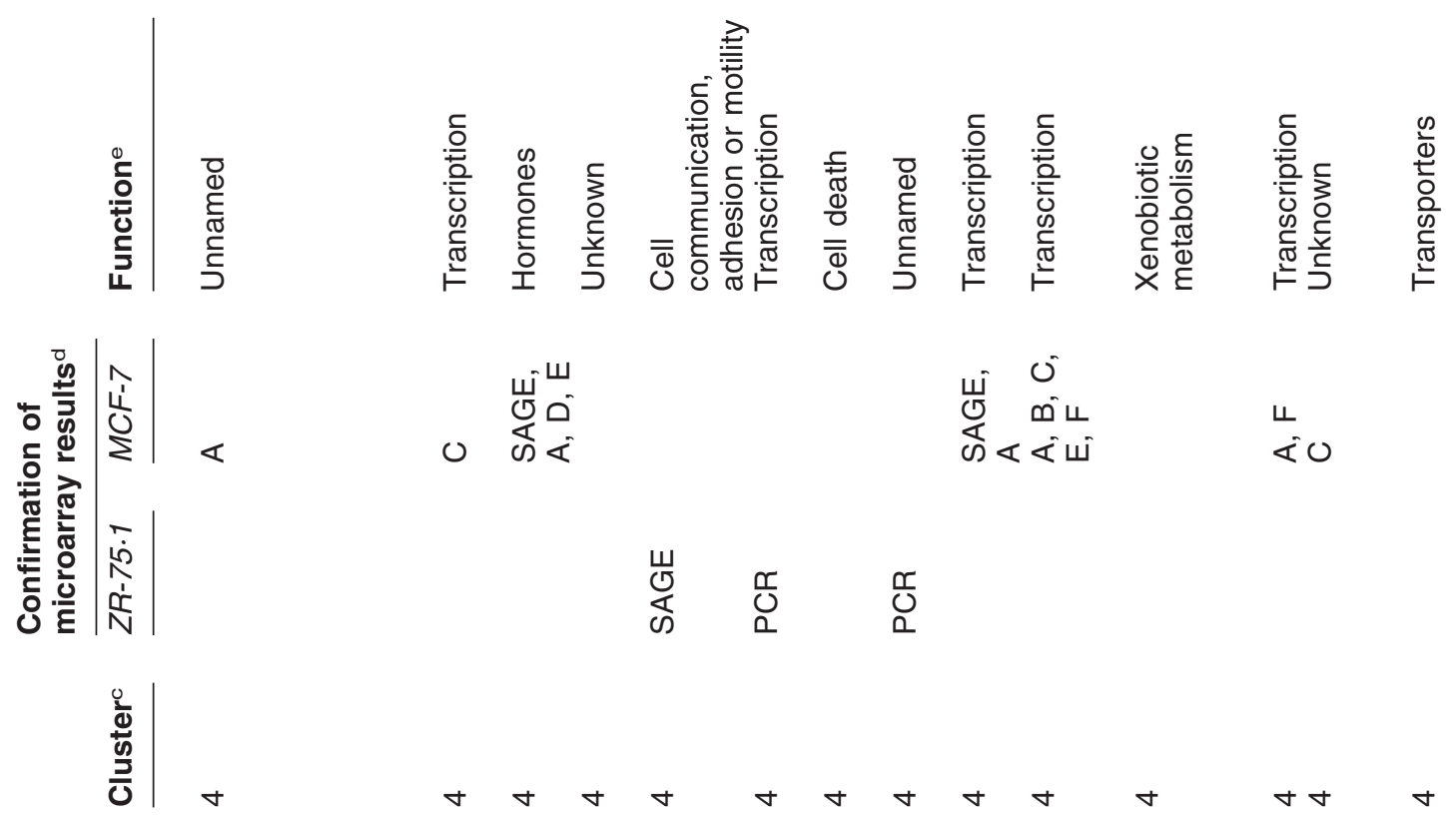

㟶总高
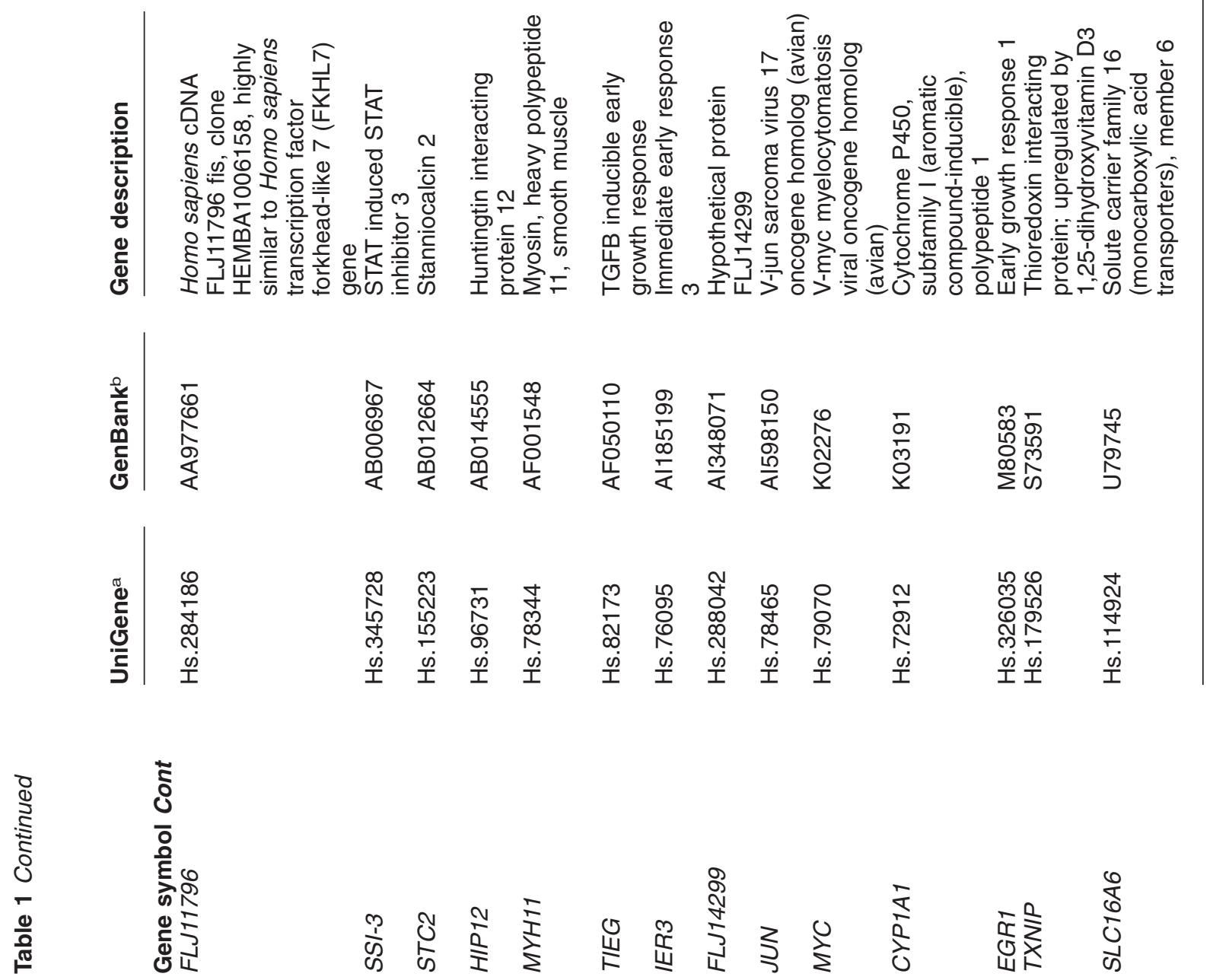

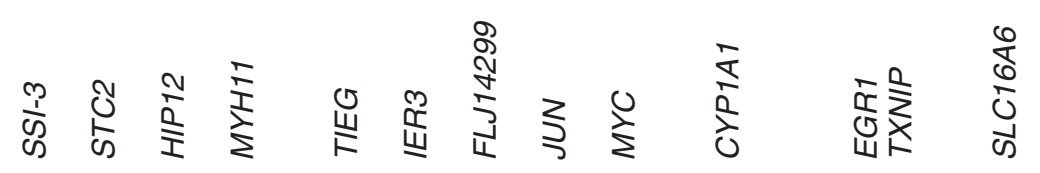



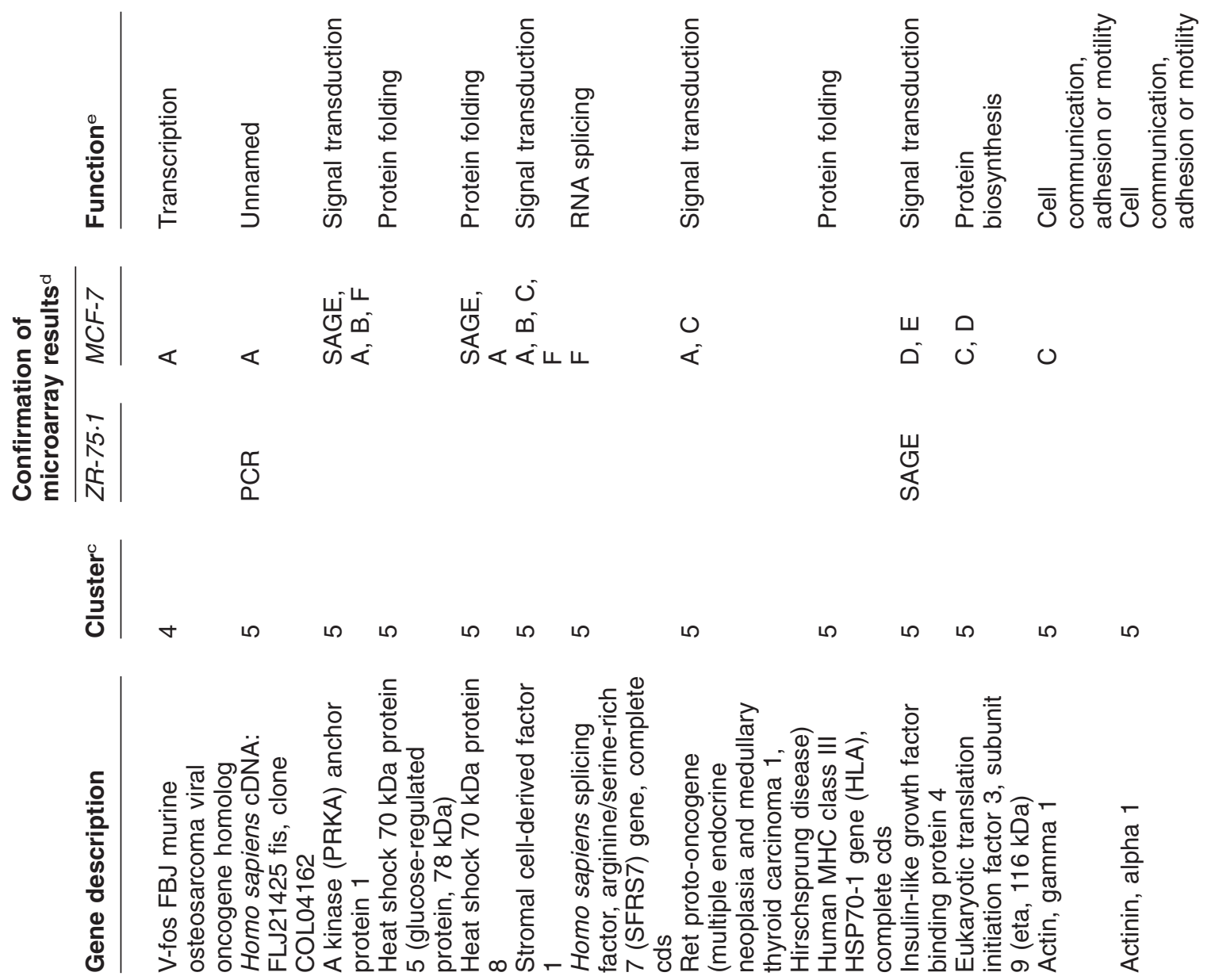

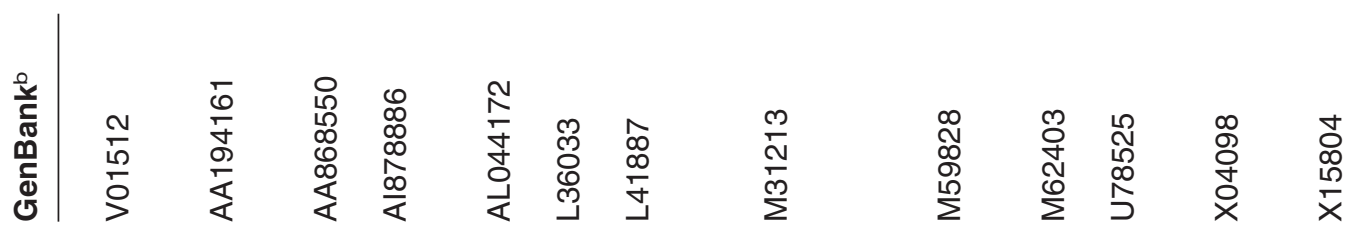

\begin{tabular}{|c|c|c|c|c|c|c|c|c|c|c|c|}
\hline 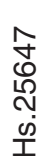 & 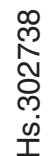 & $\begin{array}{l}\bar{N} \\
\text { D } \\
\infty \\
\text { p. } \\
\text { I }\end{array}$ & $\begin{array}{l}\text { 음 } \\
\text { م. } \\
\text { ம் } \\
\frac{0}{1}\end{array}$ & $\begin{array}{l}\frac{J}{d} \\
\dot{J} \\
\infty \\
\dot{\infty} \\
\dot{0}\end{array}$ & 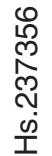 & $\begin{array}{l}\hat{6} \\
\dot{J} \\
\infty \\
\frac{0}{1} \\
\frac{0}{1}\end{array}$ & 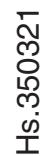 & 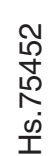 & $\begin{array}{l}\frac{0}{0} \\
\frac{\omega}{0} \\
\frac{\infty}{1}\end{array}$ & 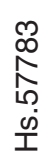 & $\begin{array}{l}0 \\
\stackrel{0}{9} \\
\stackrel{9}{ \pm} \\
\frac{\dot{\rho}}{1}\end{array}$ \\
\hline
\end{tabular}

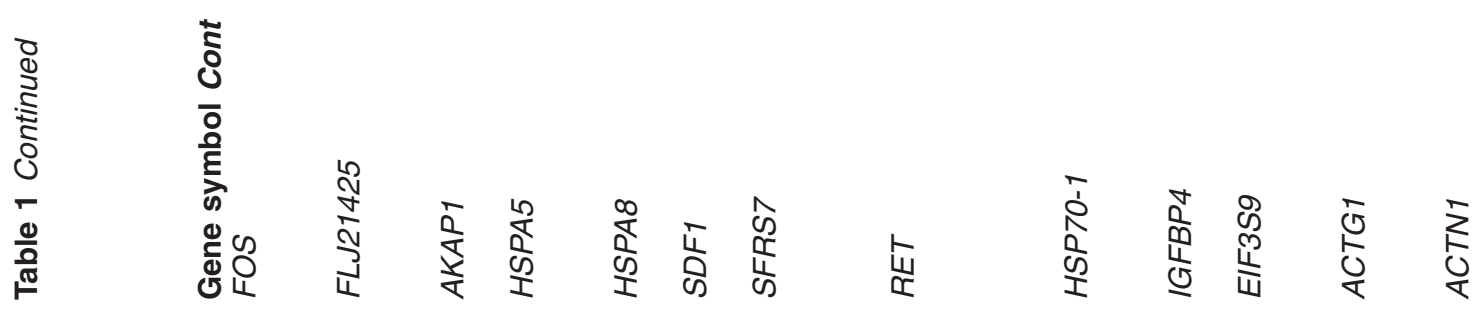



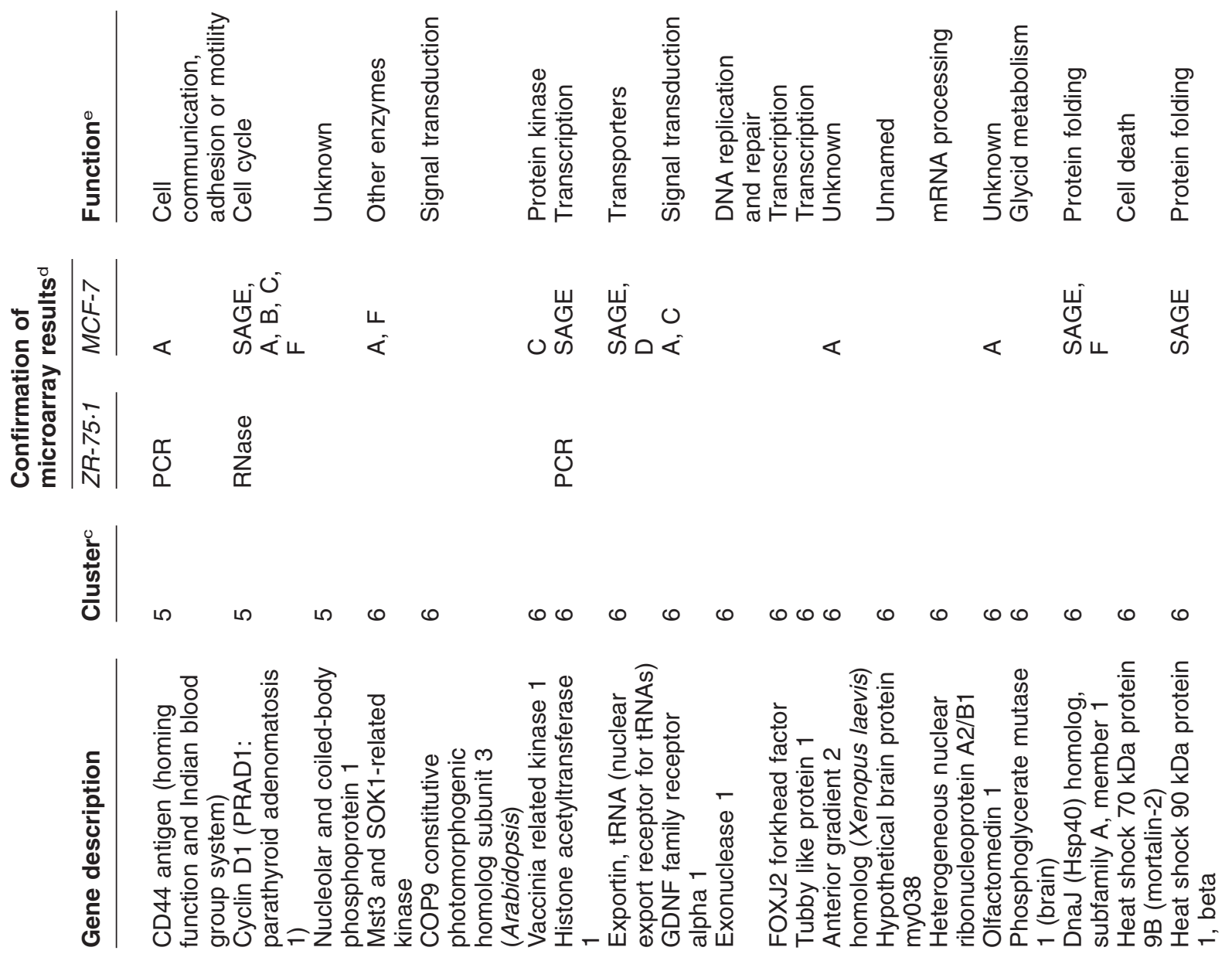

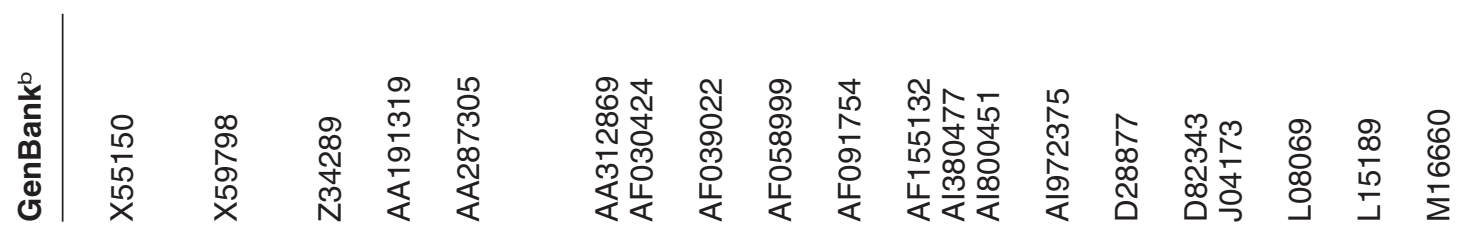

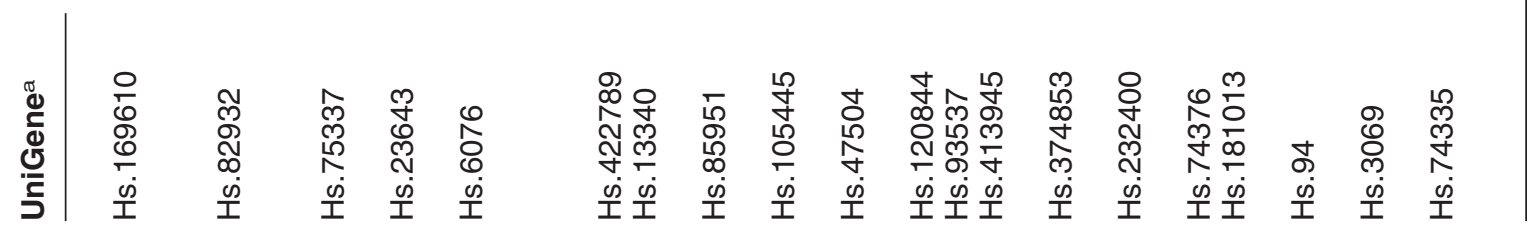

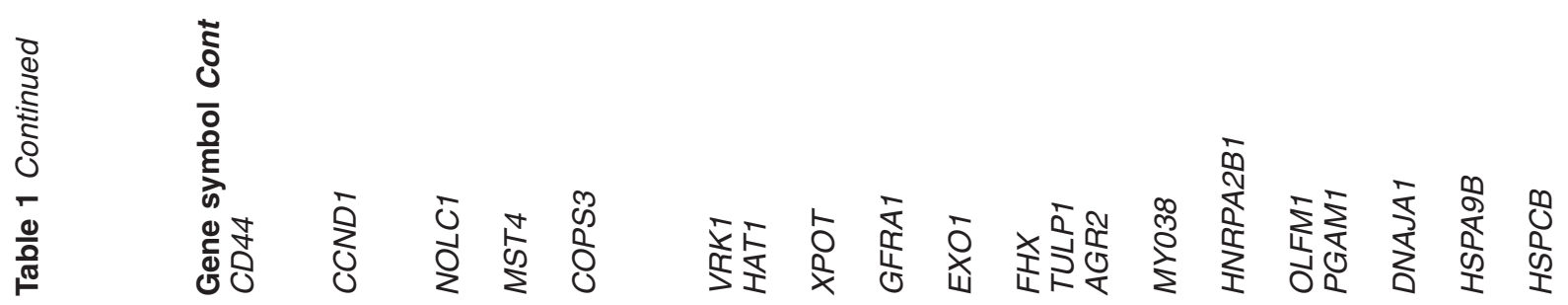




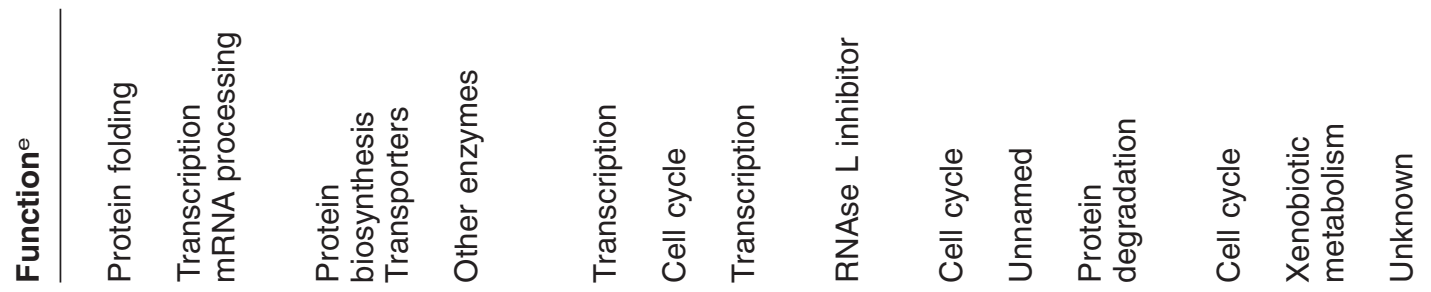

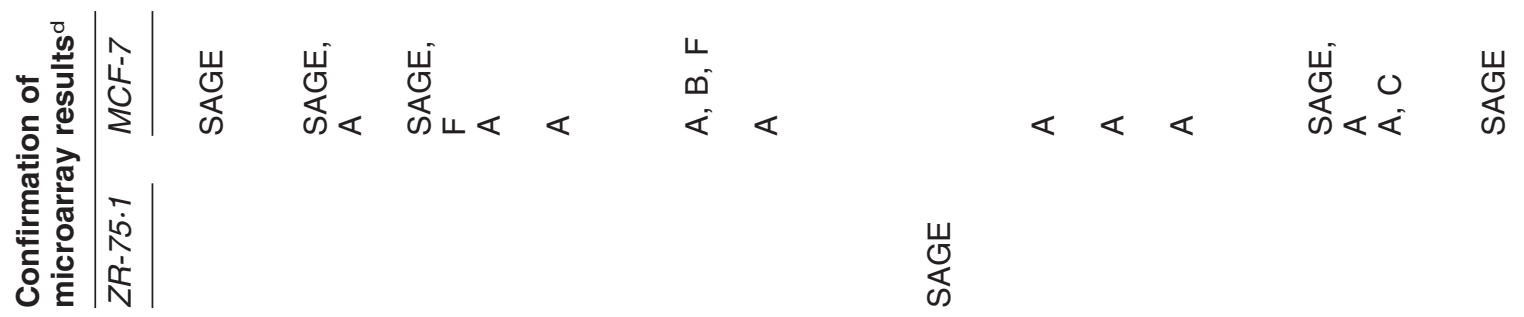

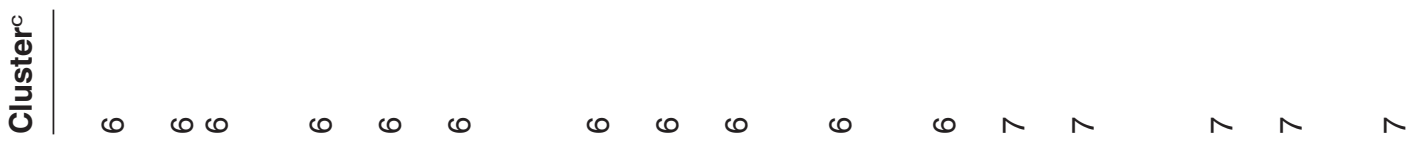

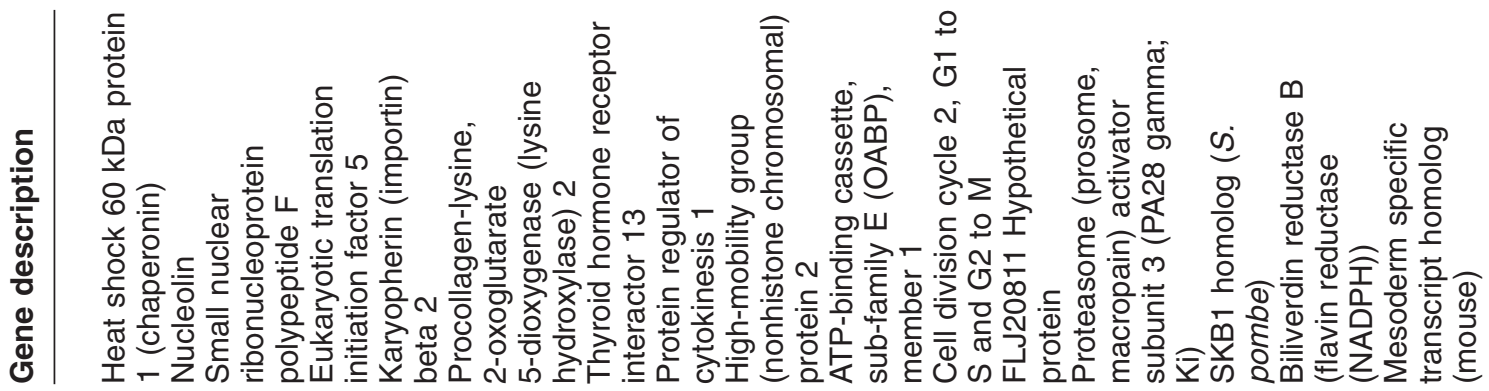

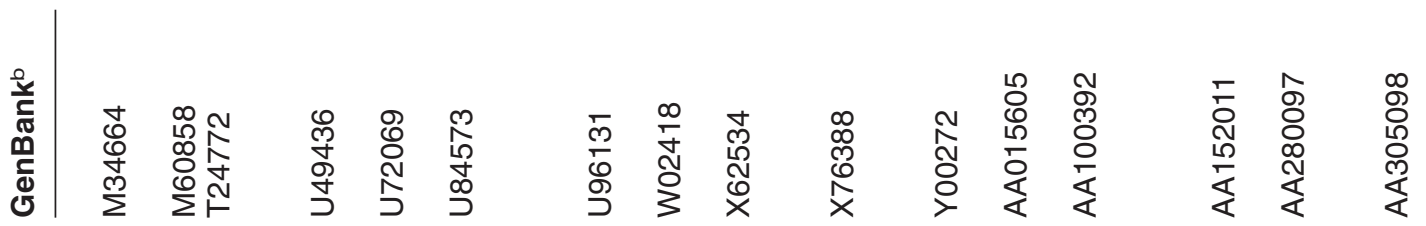

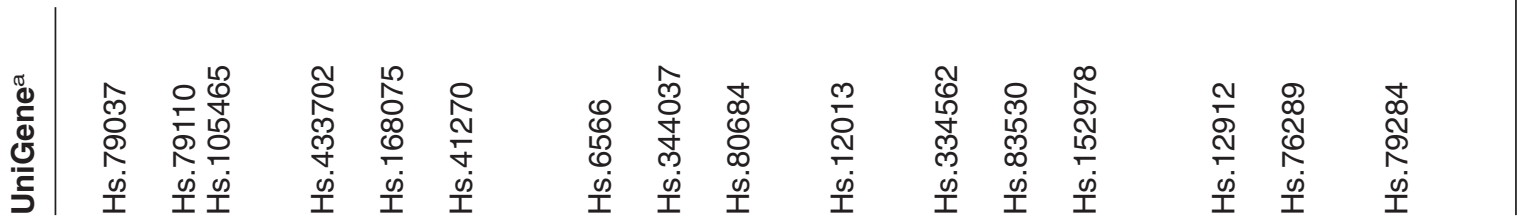

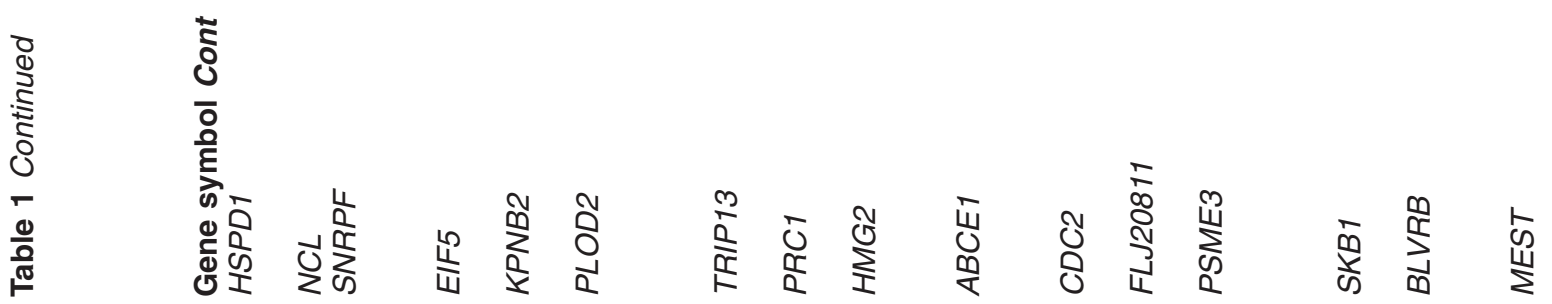



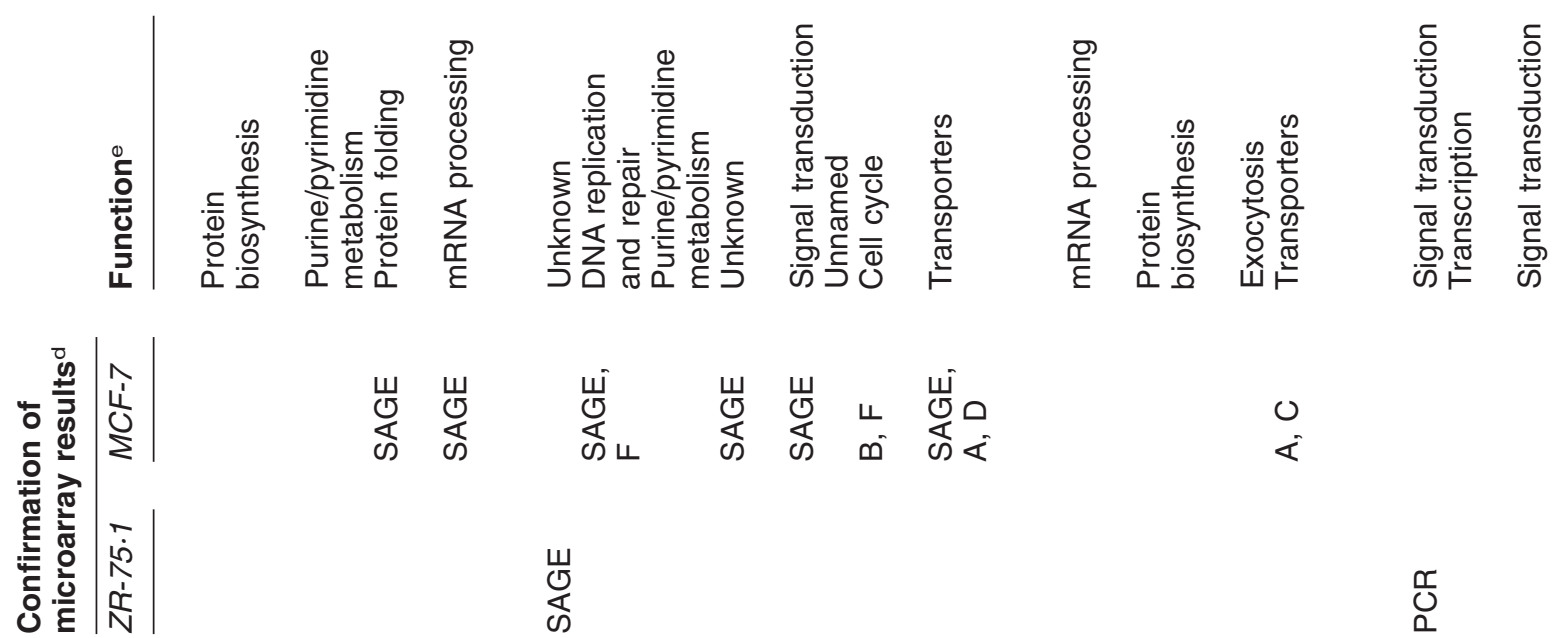

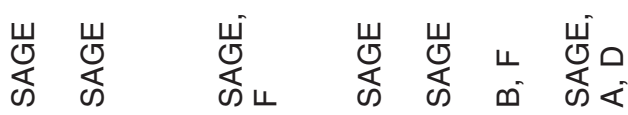

0
$\mathbb{4}$

๗

$\frac{\Upsilon 1}{0}$

$\frac{0}{0}$
$\frac{0}{0}$

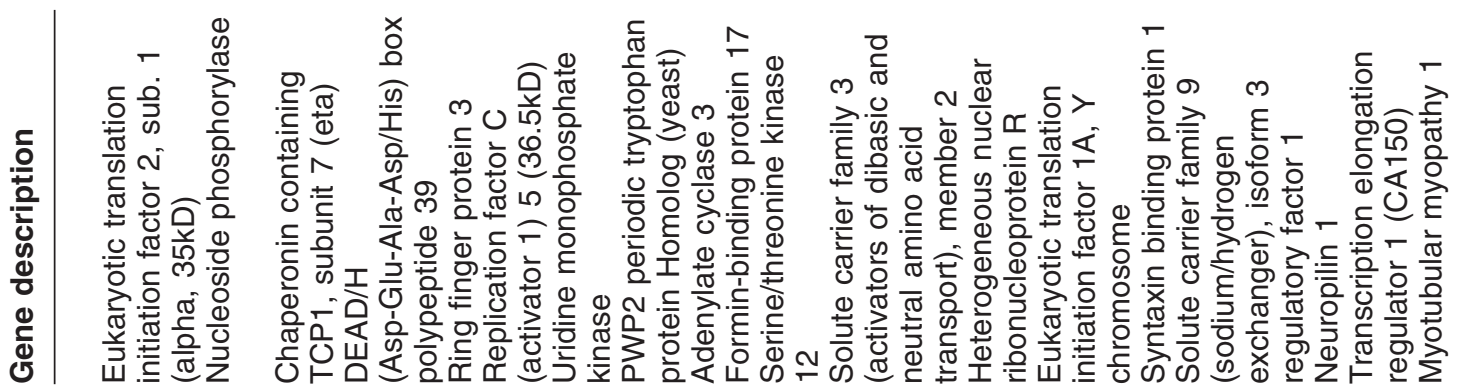

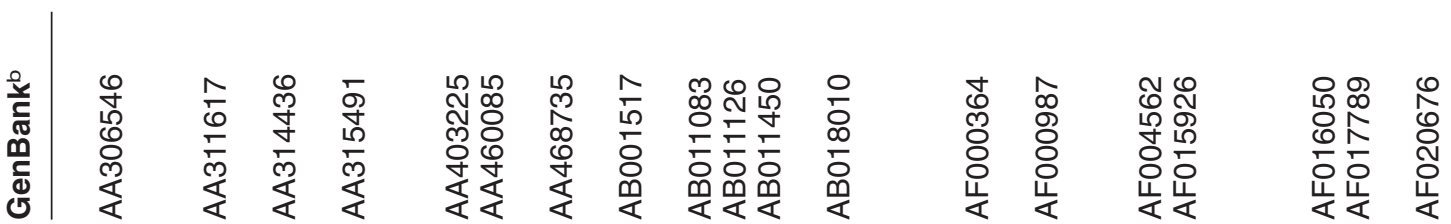

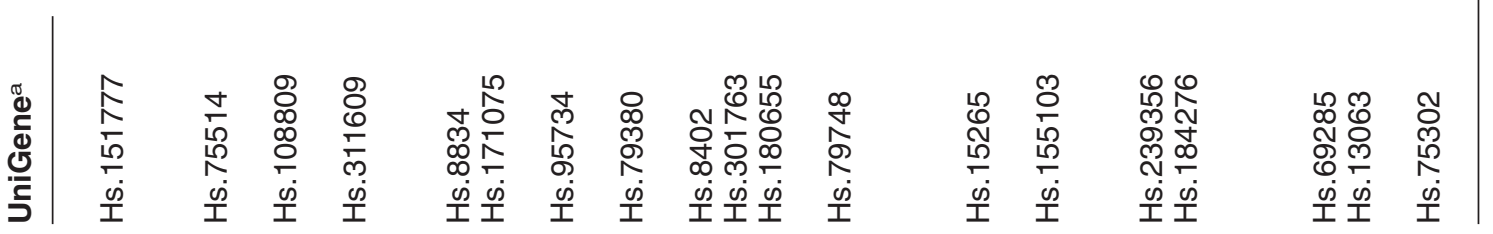

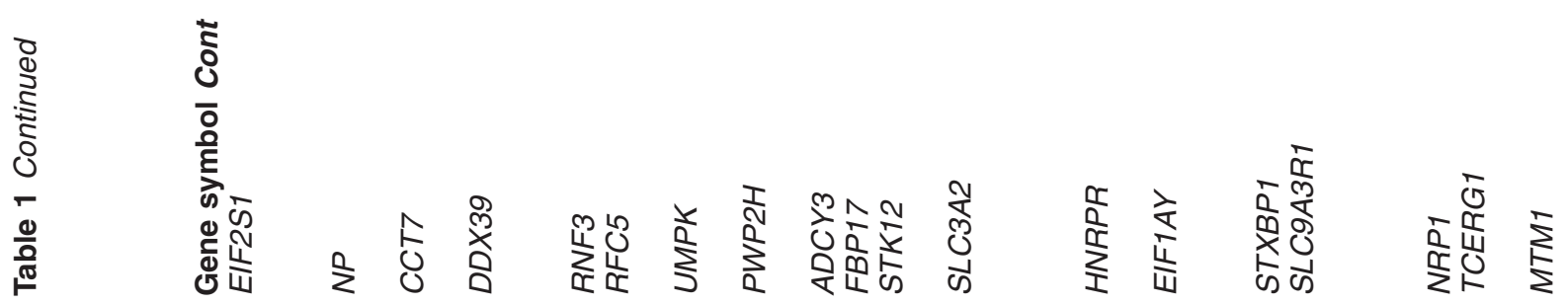



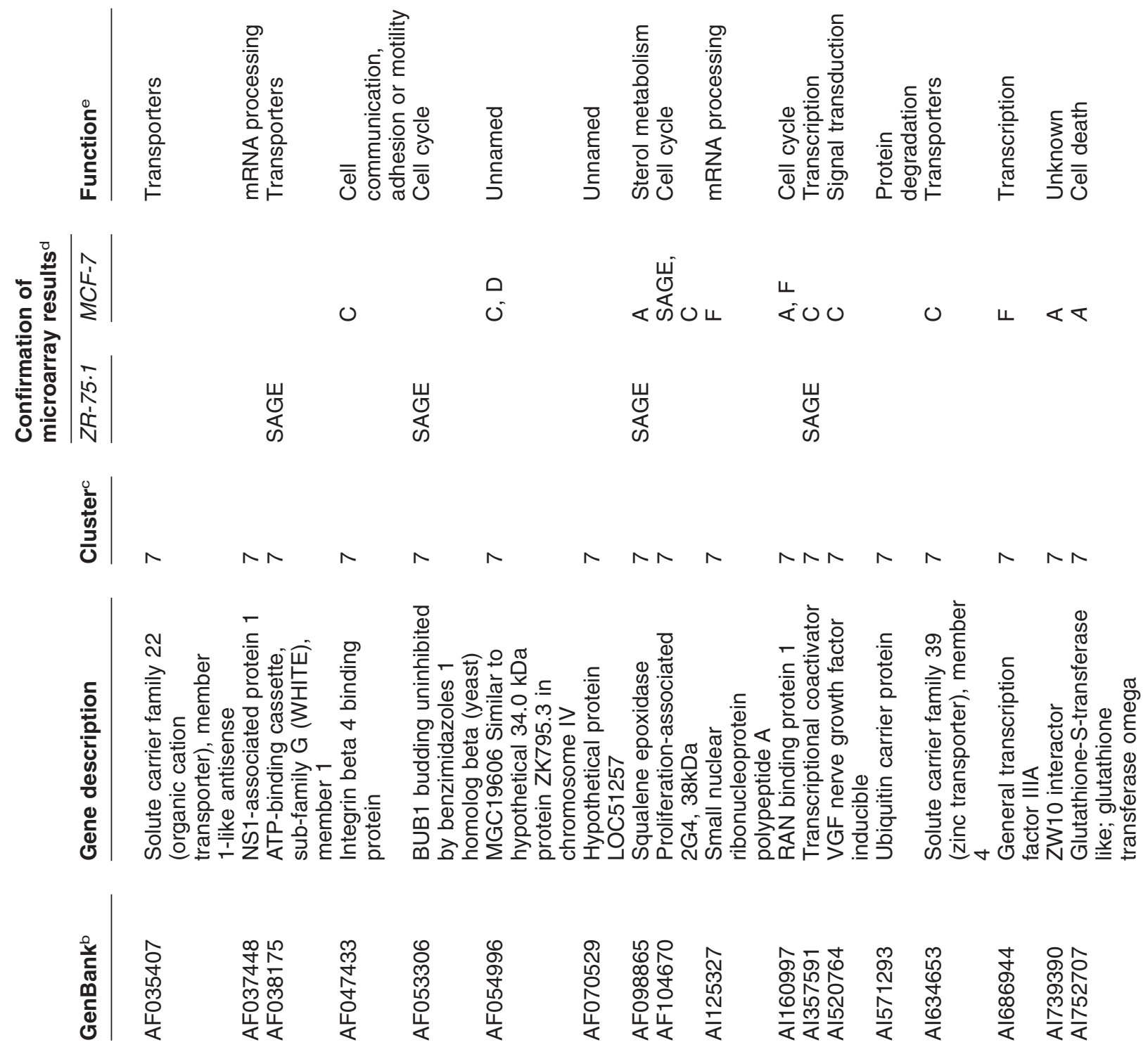

\begin{tabular}{|c|c|c|c|c|c|c|c|c|c|c|c|c|}
\hline 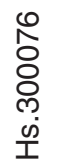 & 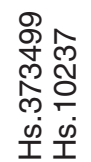 & 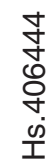 & $\begin{array}{l}\infty \\
0 \\
0 \\
0 \\
0 \\
\stackrel{0}{1}\end{array}$ & 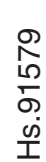 & $\begin{array}{l}\text { J } \\
\text { N } \\
\text { ल̦ } \\
\frac{\dot{0}}{1}\end{array}$ & 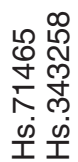 & 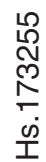 & 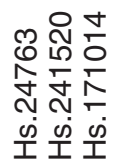 & $\begin{array}{l}\text { O } \\
\text { O } \\
\text { N } \\
\frac{1}{1} \\
\frac{0}{1}\end{array}$ & $\begin{array}{l}\frac{10}{d} \\
\text { Nิ } \\
\text { ली } \\
\stackrel{0}{1}\end{array}$ & $\begin{array}{l}\stackrel{m}{\frac{m}{n}} \\
\stackrel{n}{N} \\
\frac{\infty}{1}\end{array}$ & 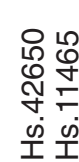 \\
\hline
\end{tabular}

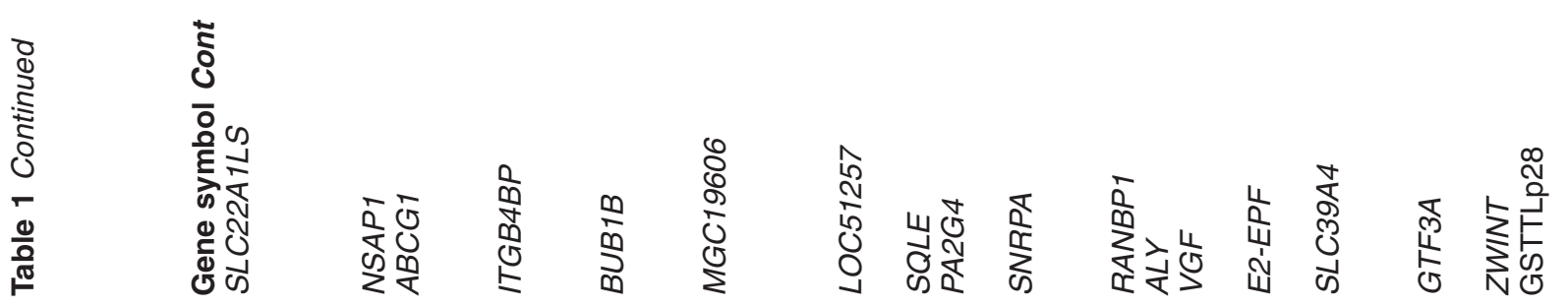




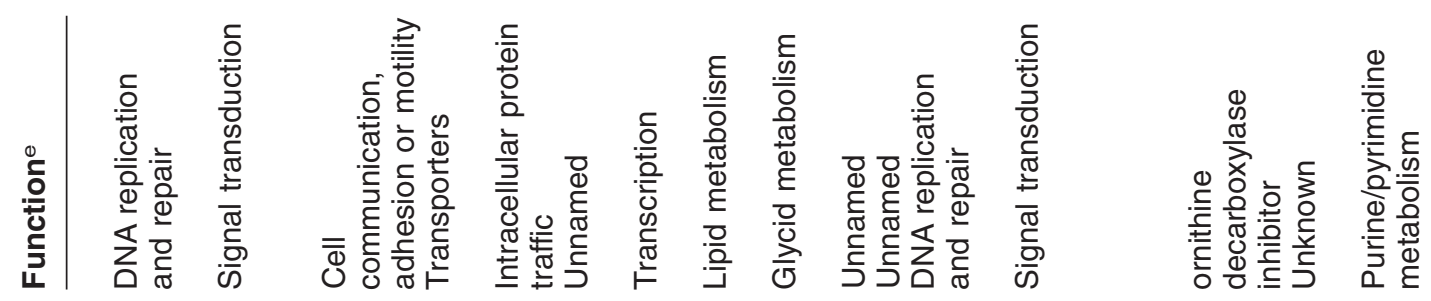
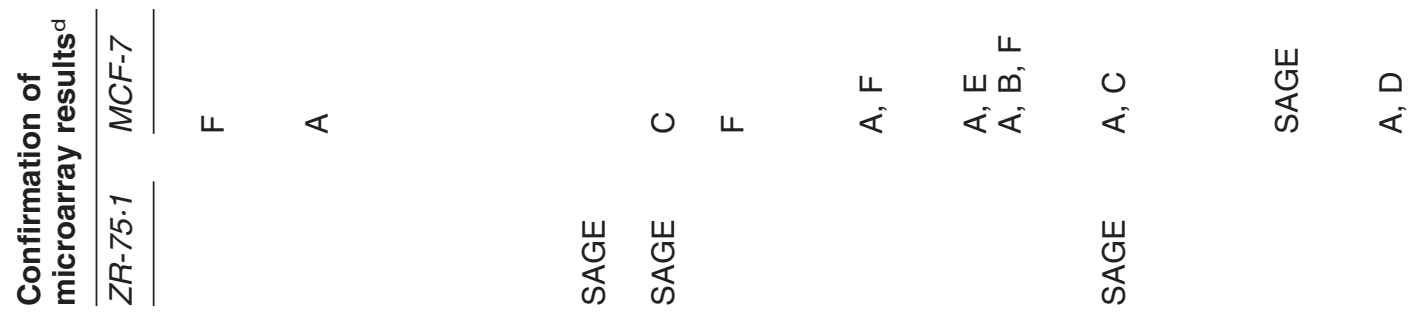

$\frac{0}{0}$
$\frac{0}{0}$

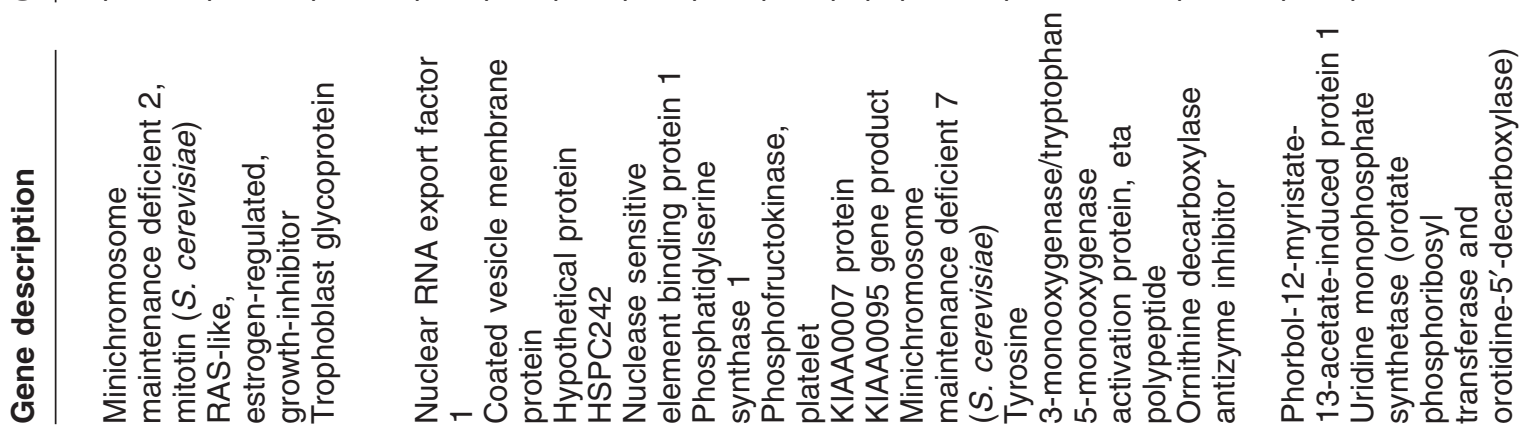

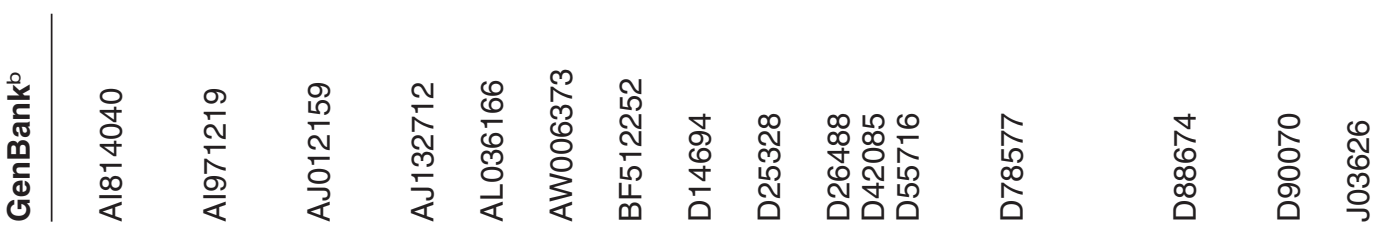

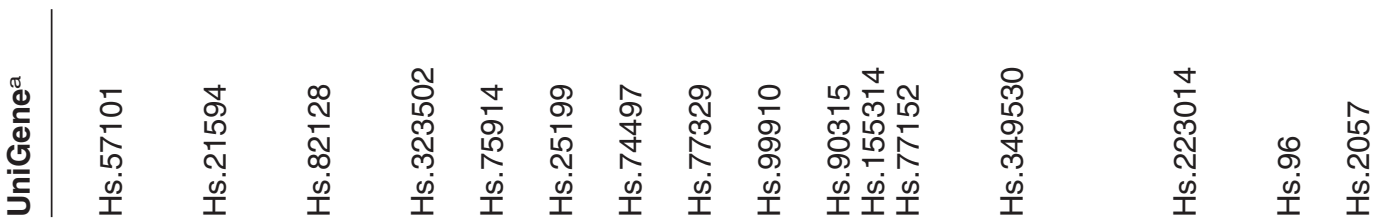

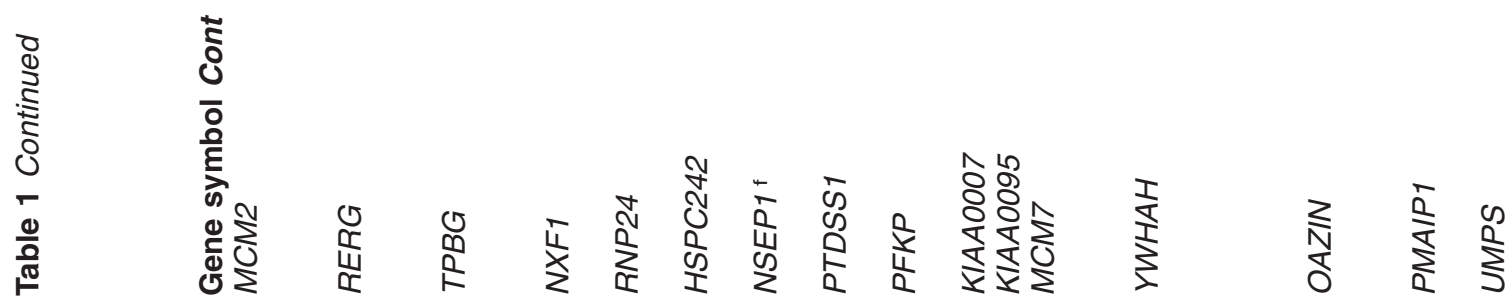



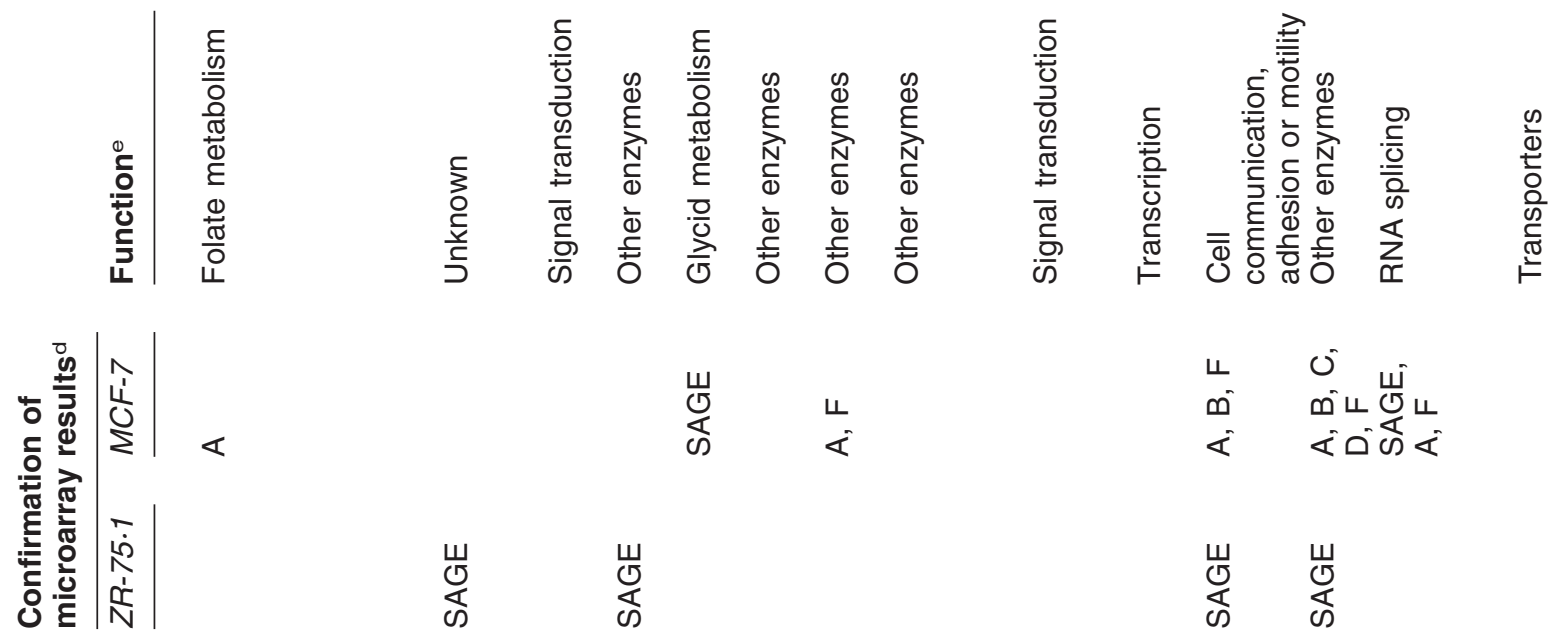

あ

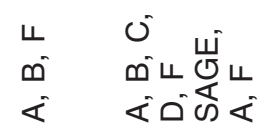

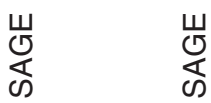

๗

N

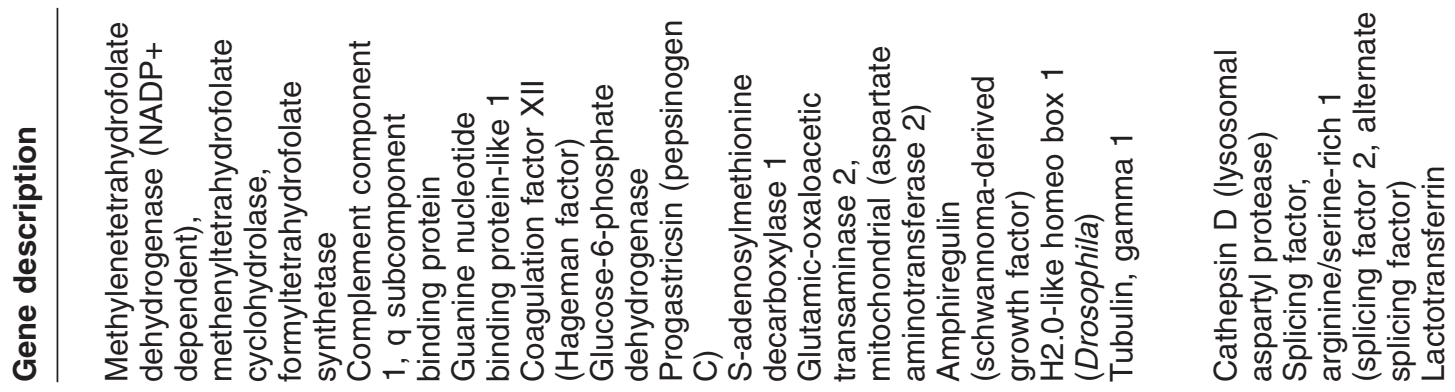

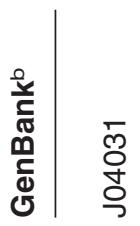

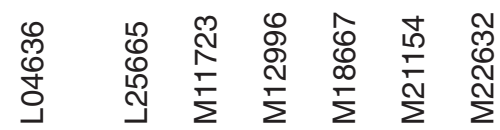

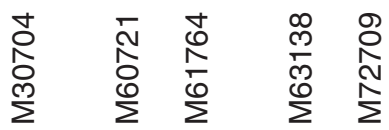

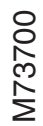

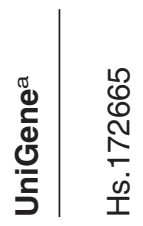

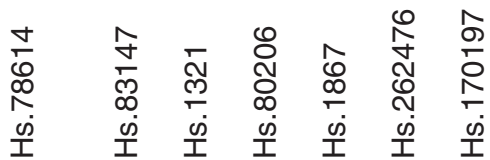

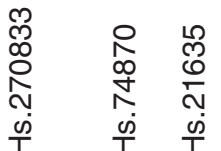

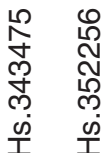

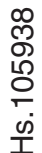

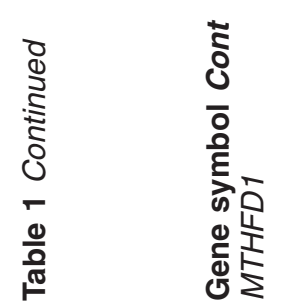

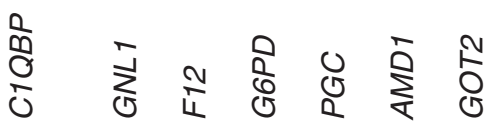

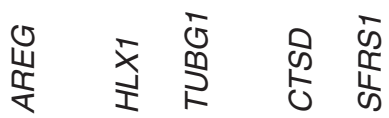

$\stackrel{4}{5}$ 


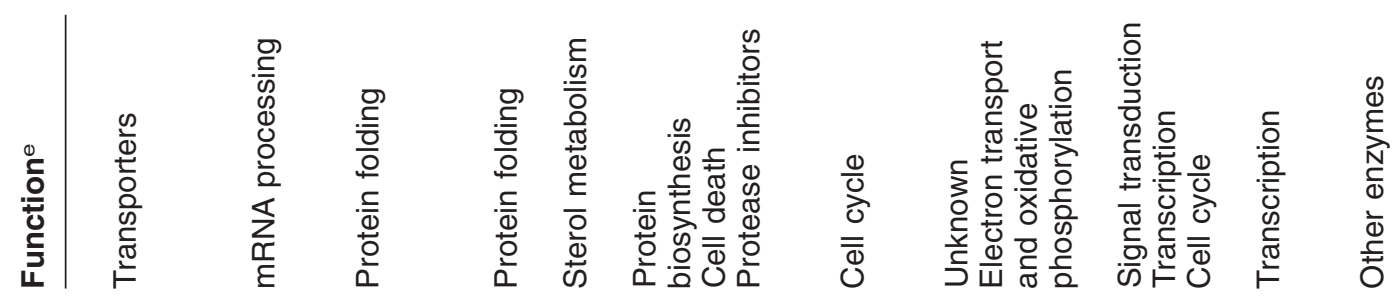

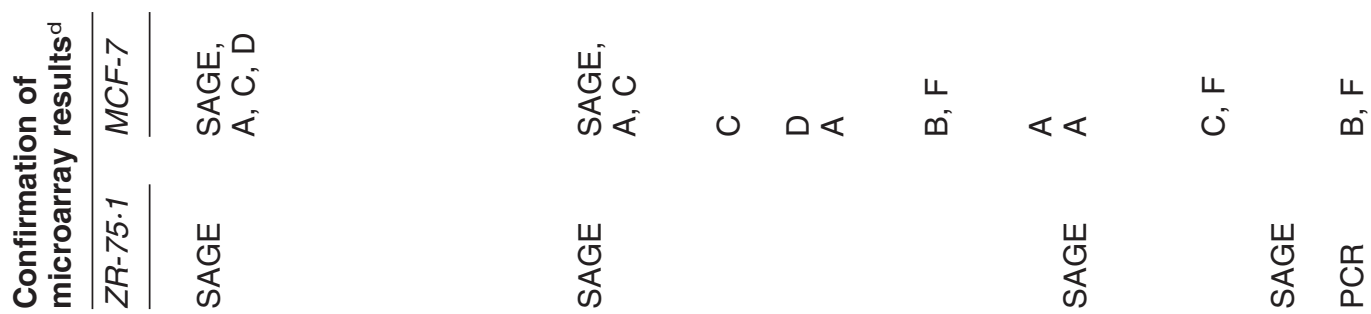

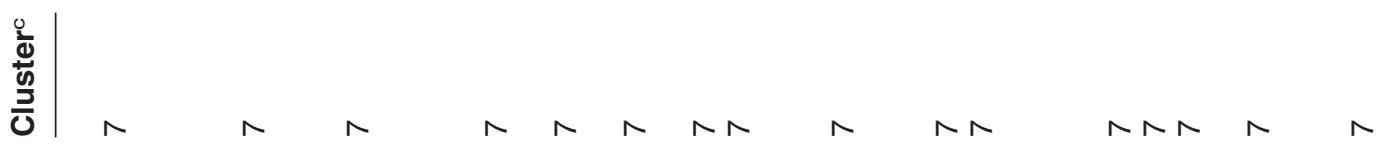

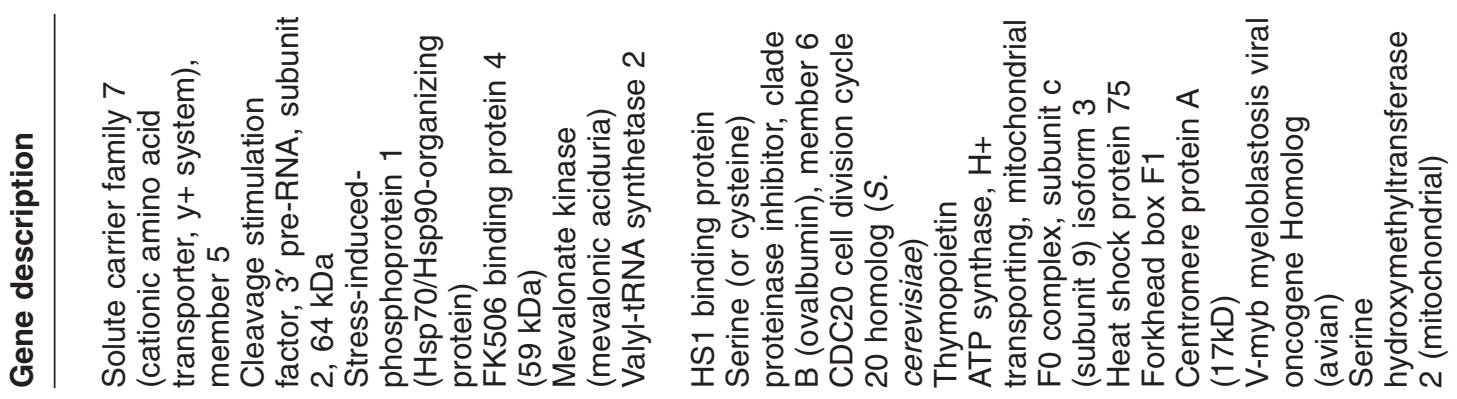

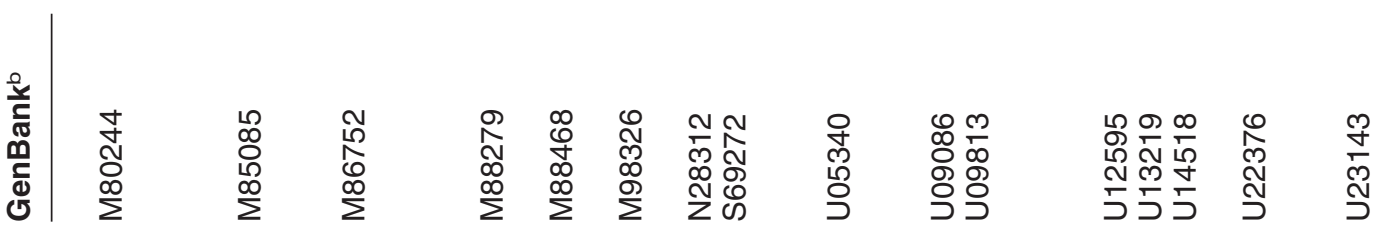

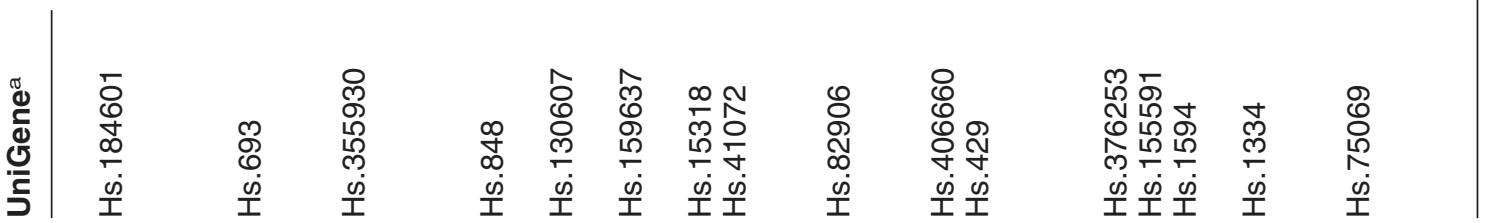

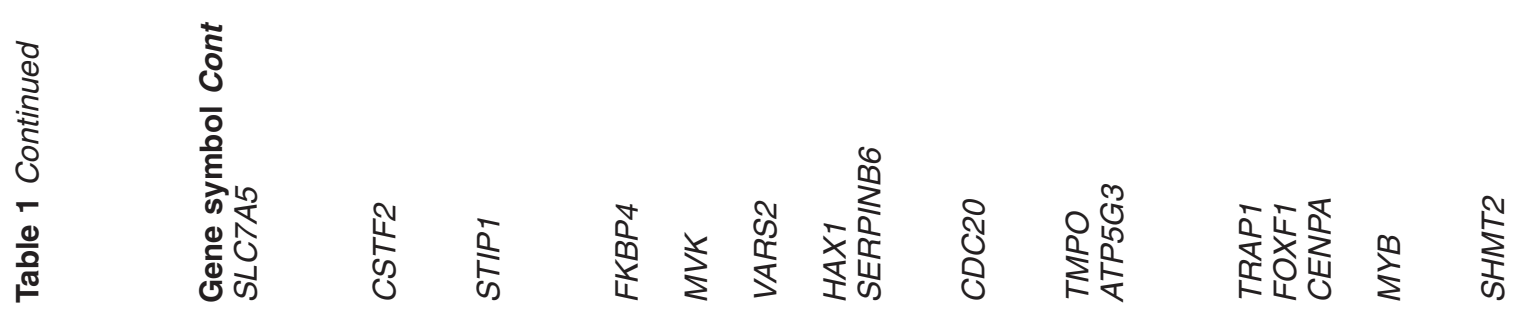




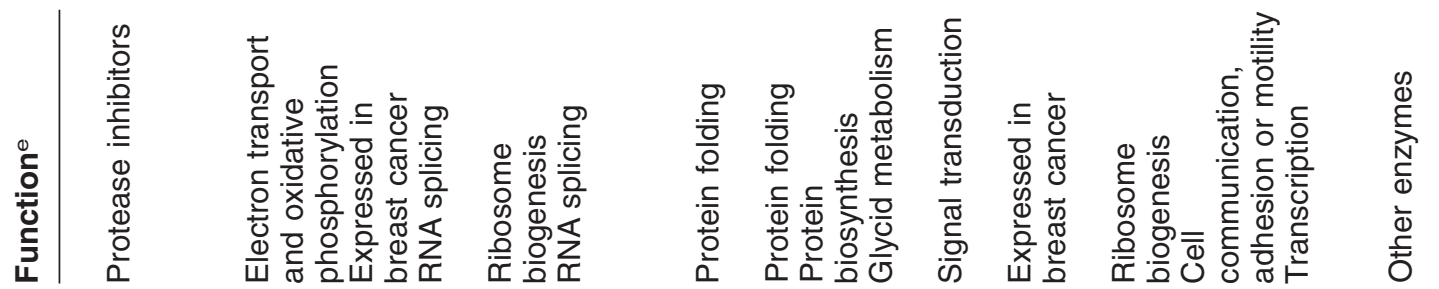

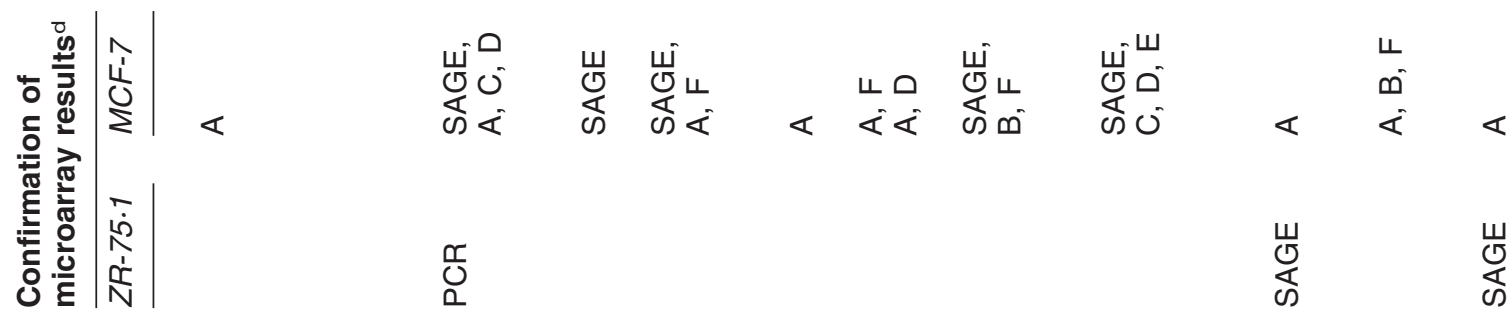

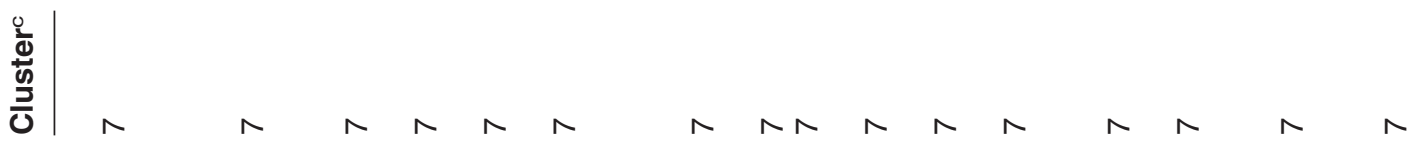

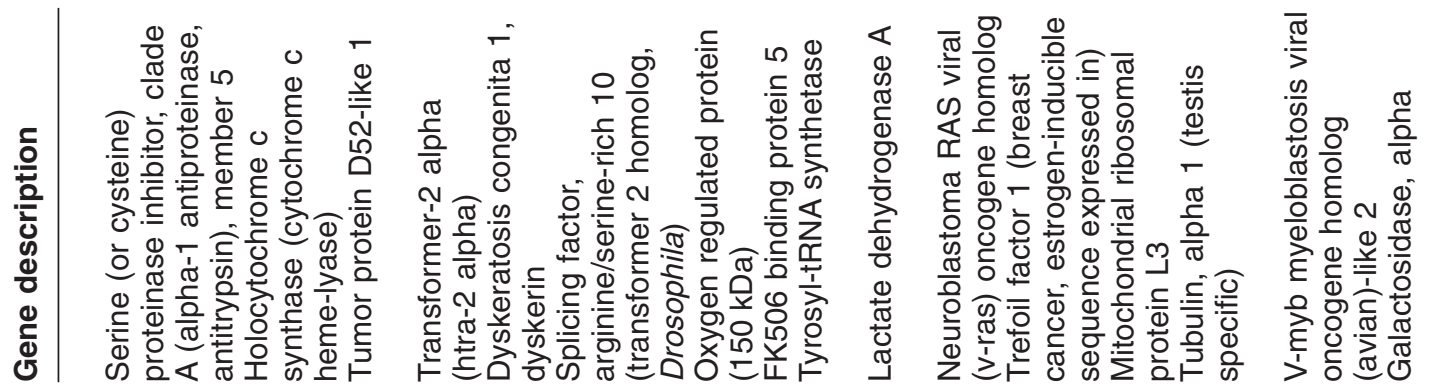

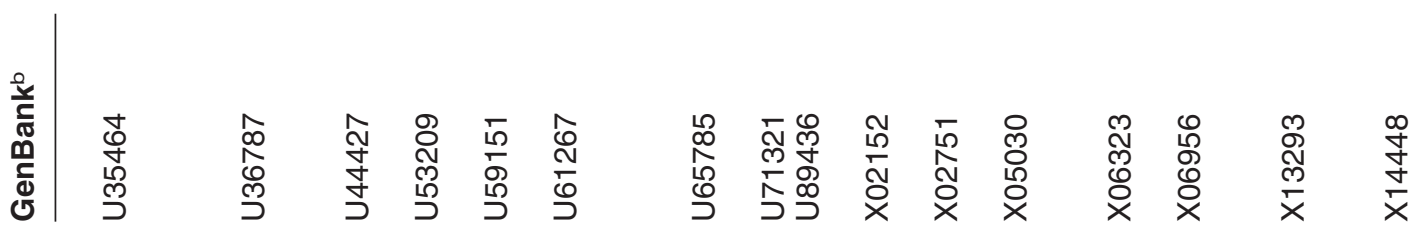

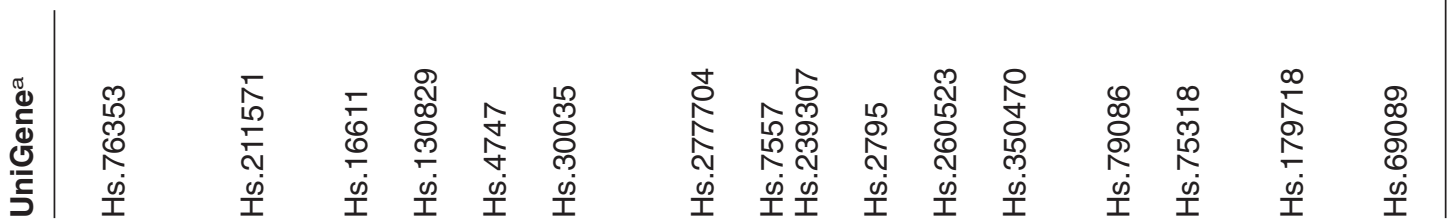

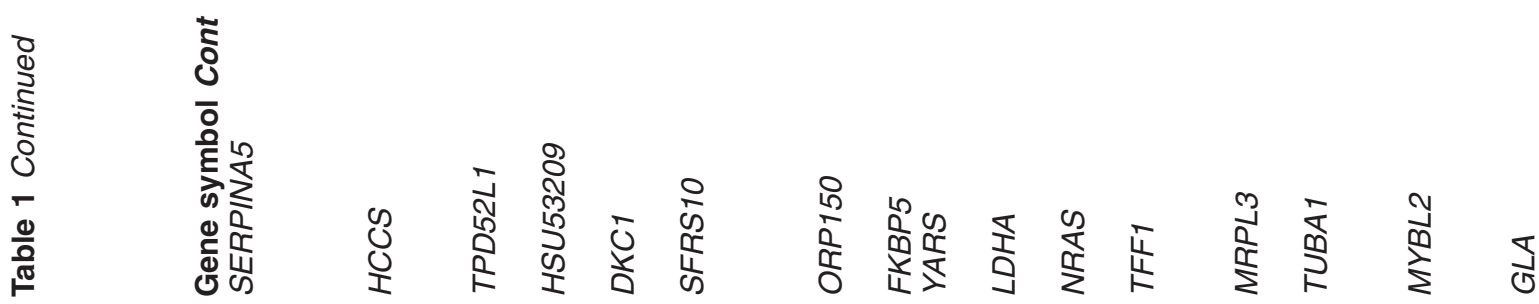



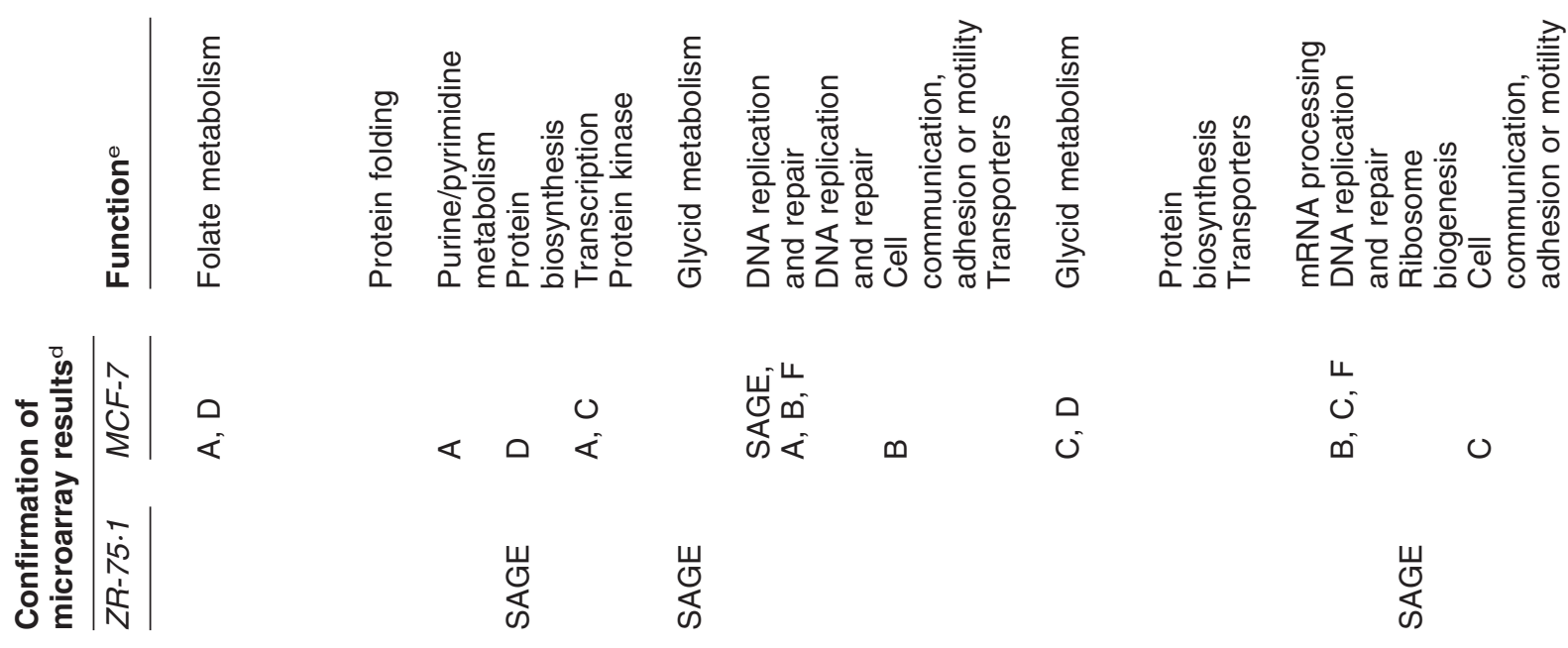

$\begin{array}{lll} & 4 & \\ 0 & j & \\ 0 & 0 & 0\end{array}$
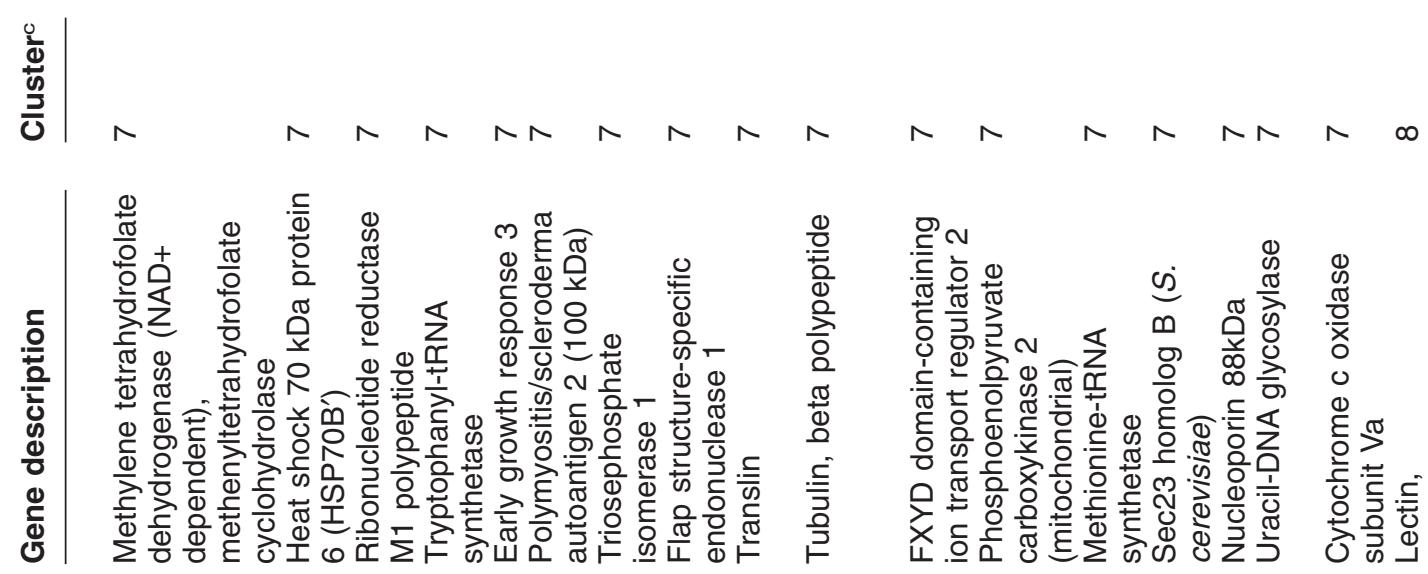

๗
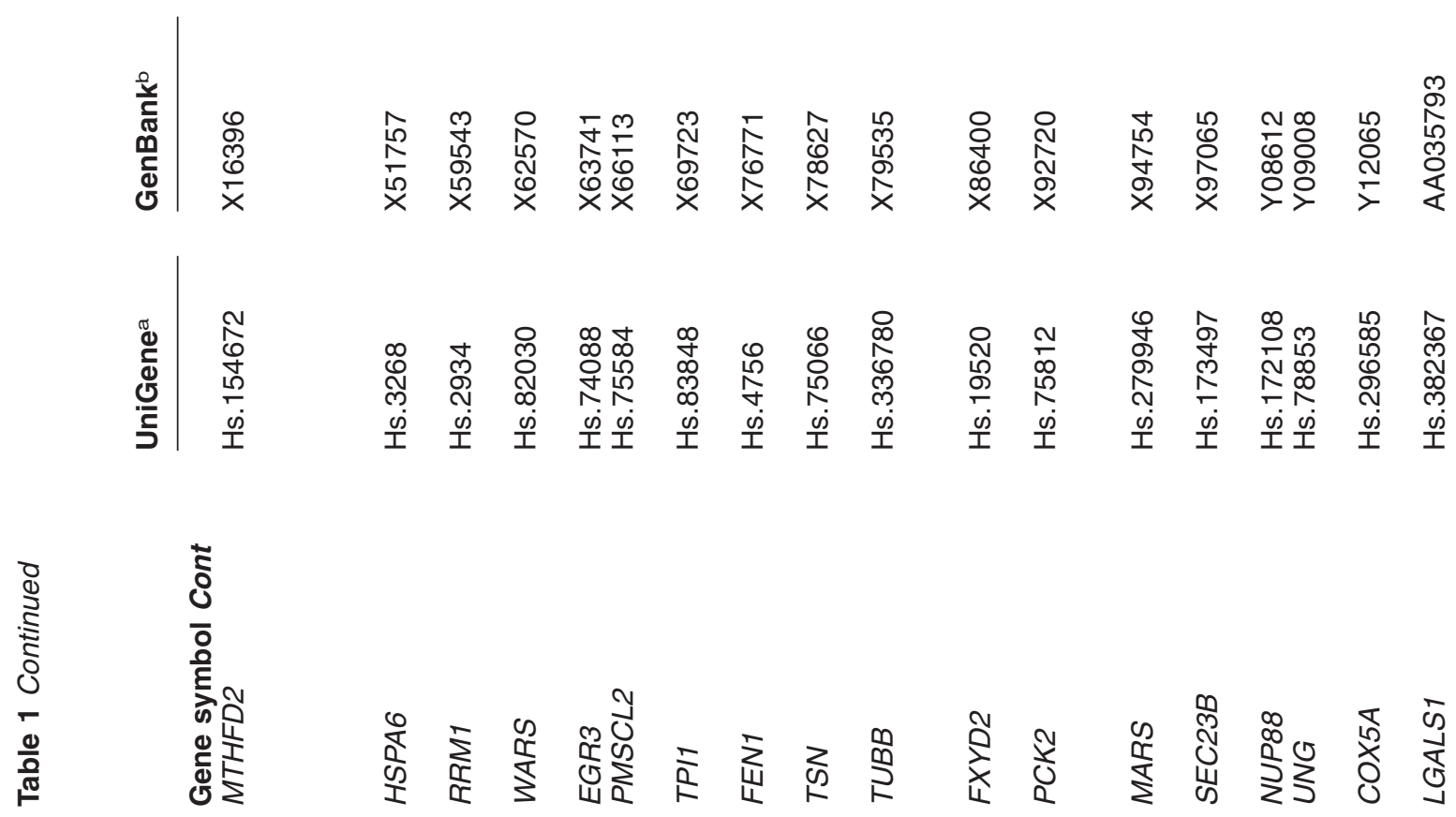

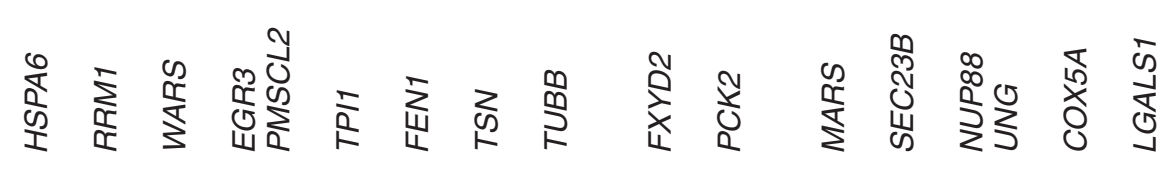




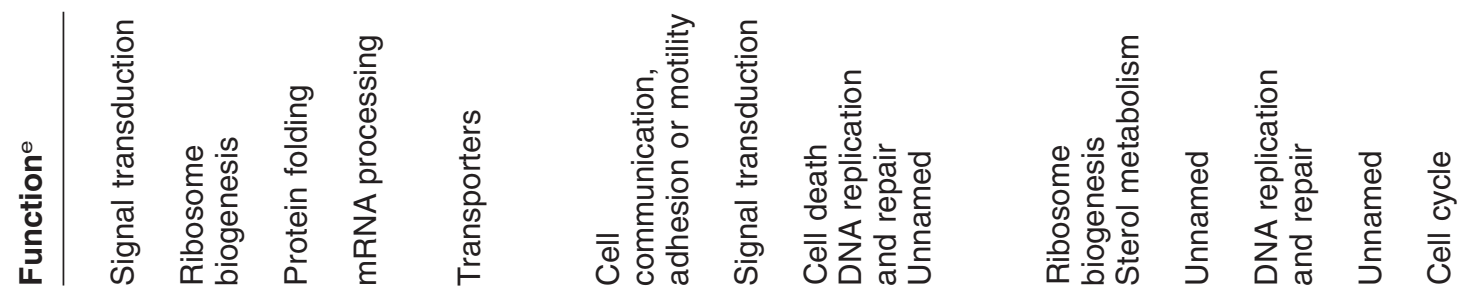
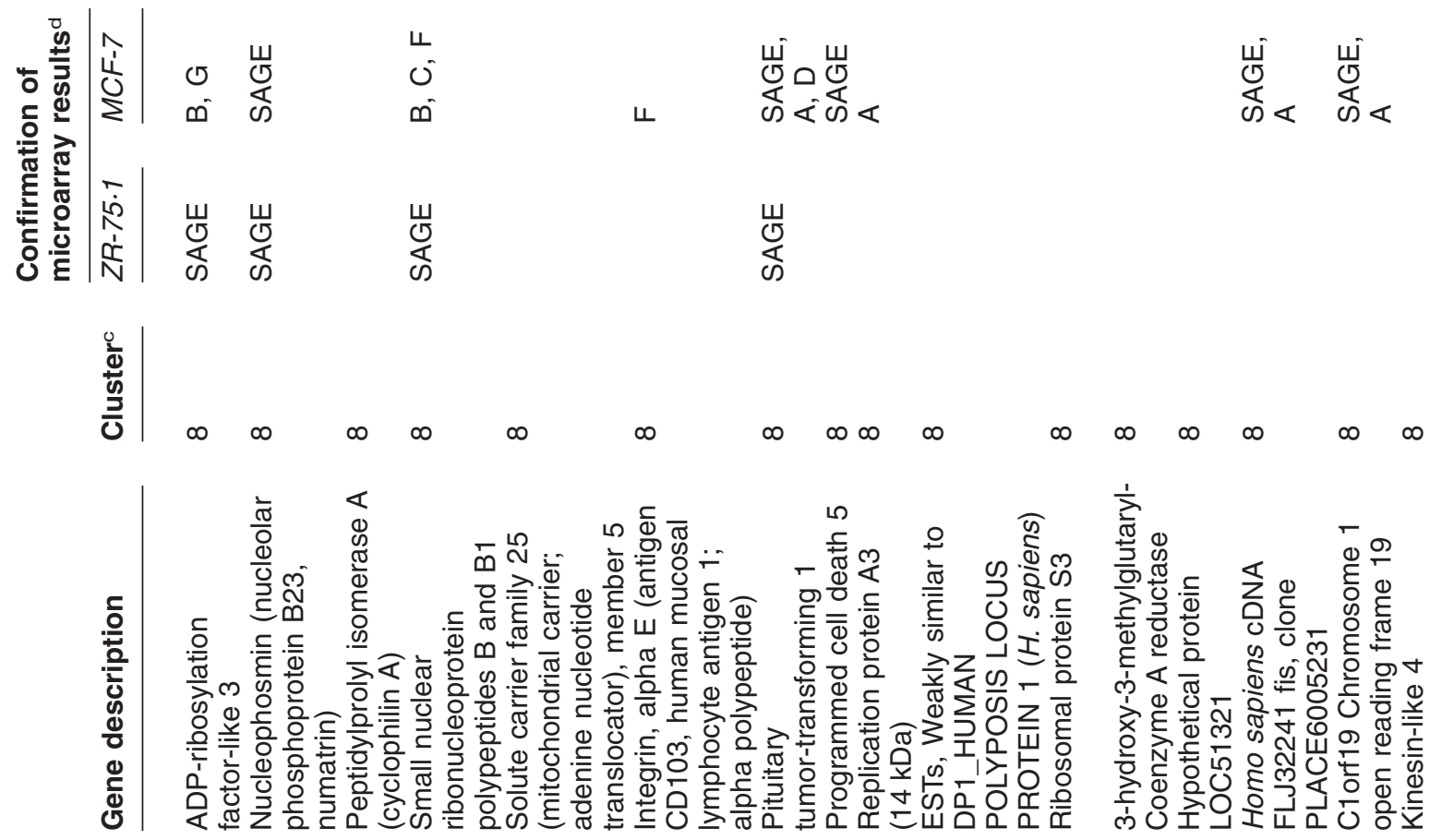

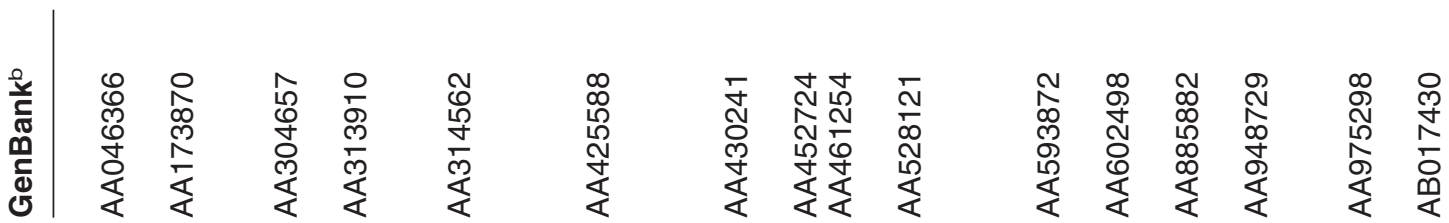

\begin{tabular}{|c|c|c|c|c|c|c|c|c|c|c|c|c|c|c|}
\hline $\begin{array}{l}\frac{1}{\sim} \\
\text { N̦ } \\
\infty \\
\frac{\infty}{1} \\
\frac{\infty}{1}\end{array}$ & 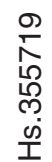 & 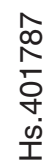 & 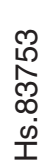 & $\begin{array}{l}\frac{N}{N} \\
\frac{\sigma}{N} \\
\frac{\infty}{1}\end{array}$ & $\begin{array}{l}\bar{n} \\
\infty \\
\dot{0} \\
\overline{1}\end{array}$ & $\begin{array}{l}\hat{\infty} \\
\stackrel{N}{N} \\
\hat{N} \\
N \\
\stackrel{0}{1}\end{array}$ & 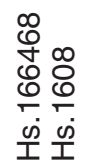 & 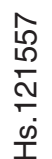 & $\begin{array}{l}\text { O } \\
\text { D } \\
\frac{1}{+} \\
\dot{0} \\
\frac{0}{1}\end{array}$ & 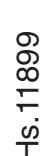 & 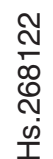 & 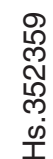 & 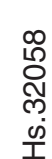 & 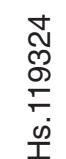 \\
\hline
\end{tabular}

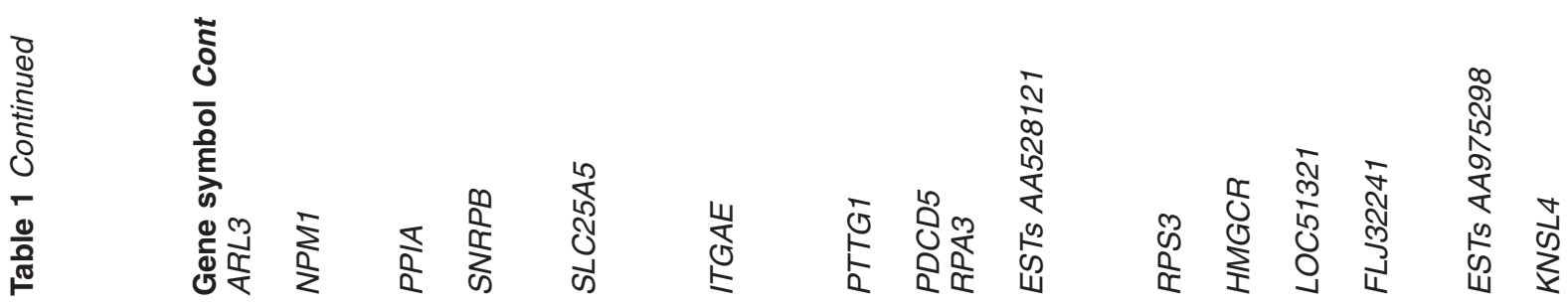




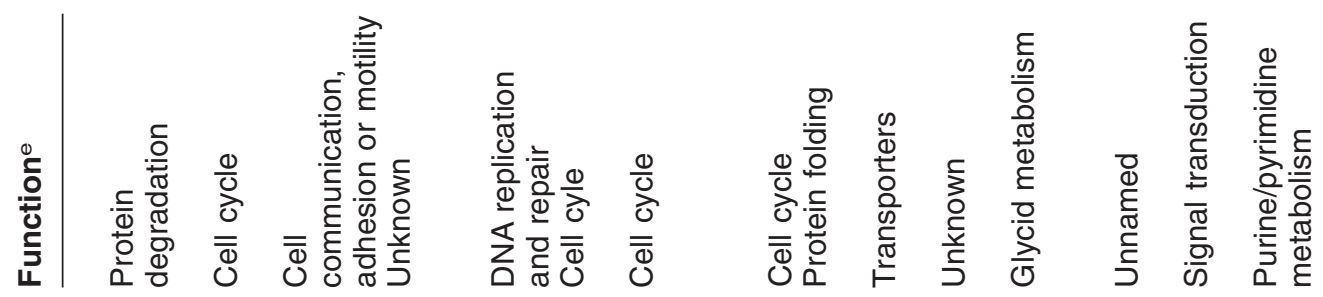

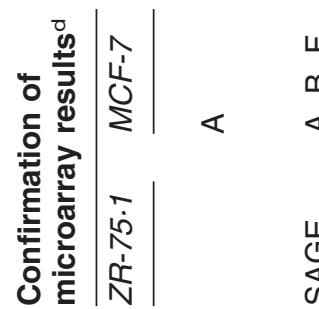

レ

岕

岕

뜽

ङ

$\frac{0}{\frac{0}{0}}$

$\infty \quad \infty \quad \infty \quad \infty \quad \infty \quad \infty \quad \infty \quad \infty$

$\infty \infty \infty \quad \infty \quad \infty \quad \infty \quad \infty \quad \infty \quad \infty$

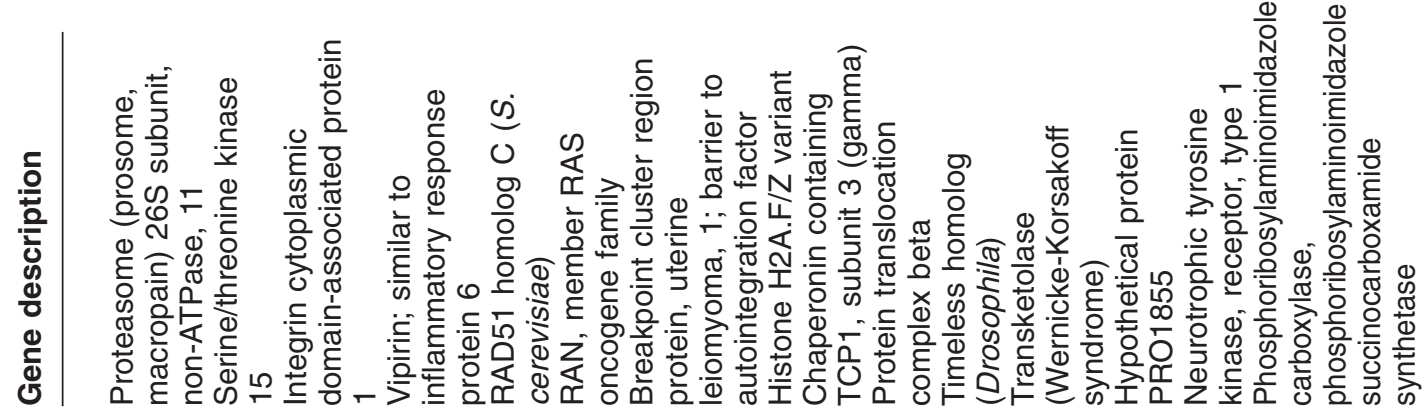

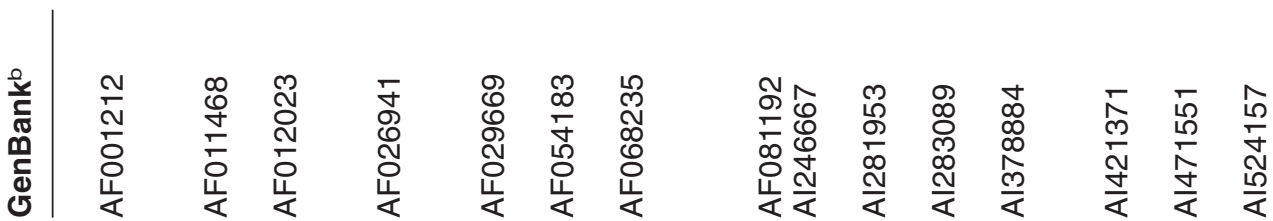

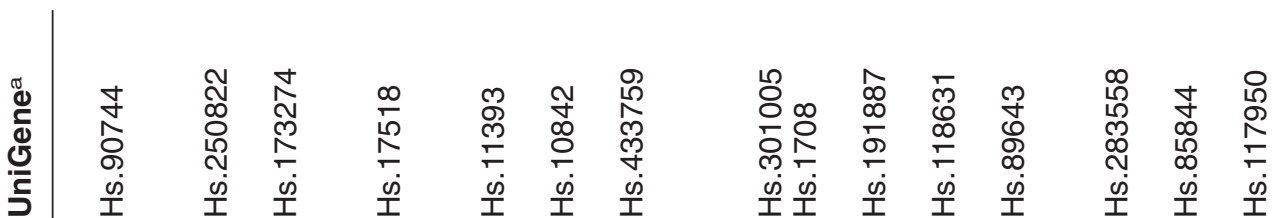

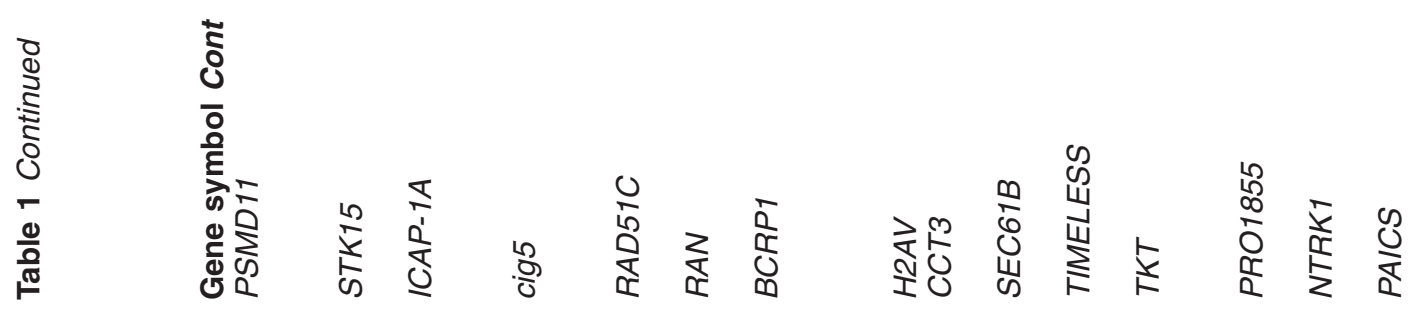




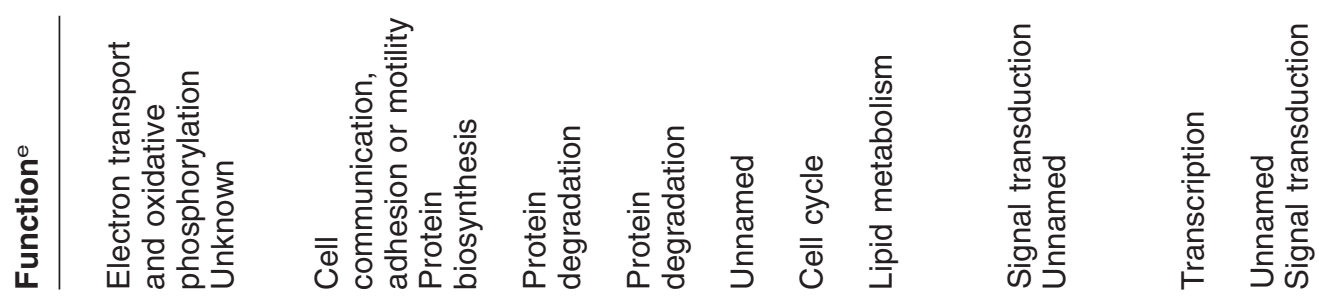

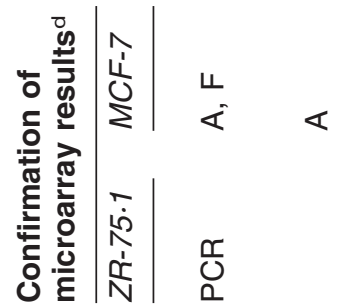

这

$\infty \quad \infty$

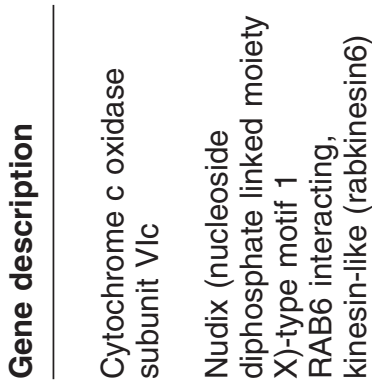

凹

๗

๓

๗

$\leftarrow \quad \varangle \varangle$

๗

๗

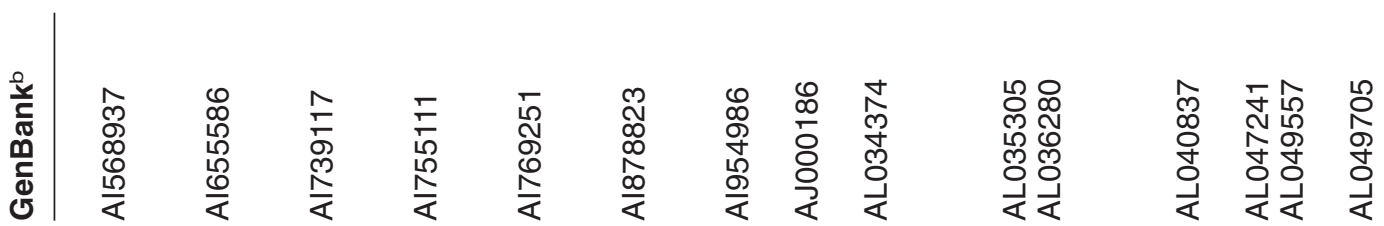

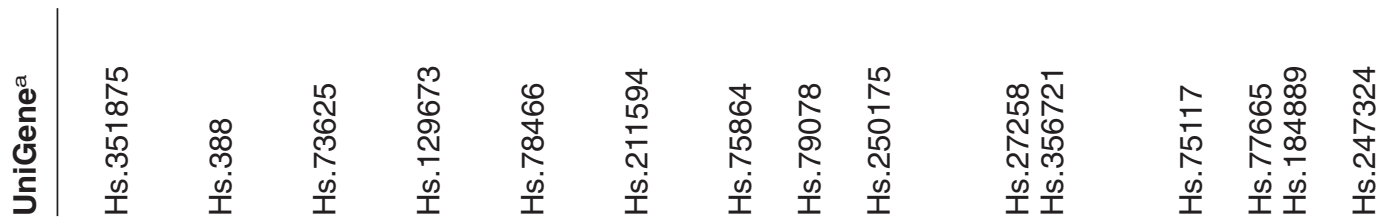

ป

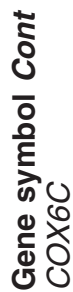

₹

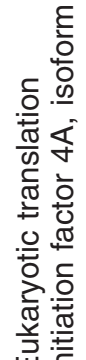

$\infty \quad \infty \quad \infty \quad \infty \quad \infty$

$\infty \infty$

$\infty \quad \infty \infty \infty$

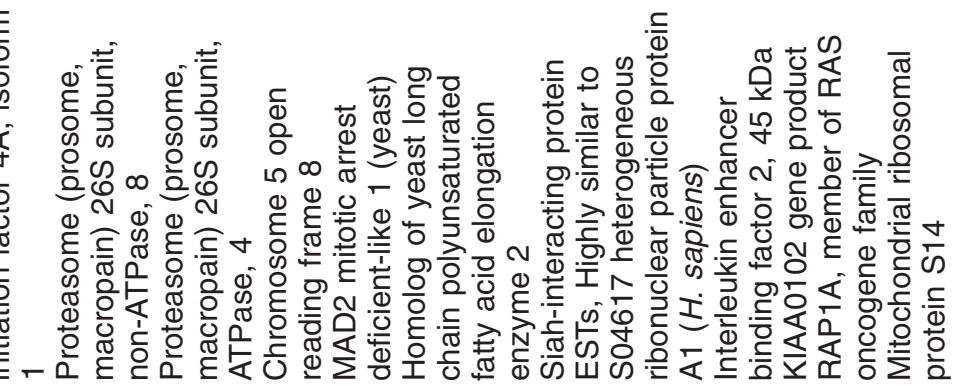




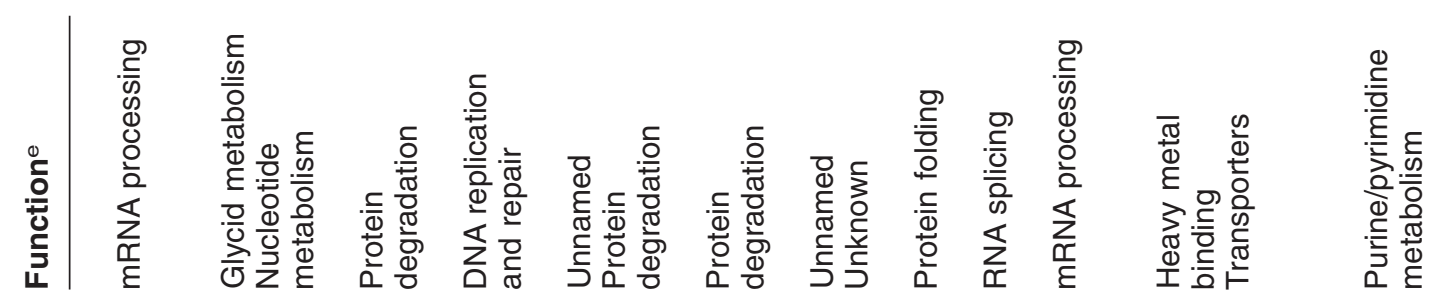
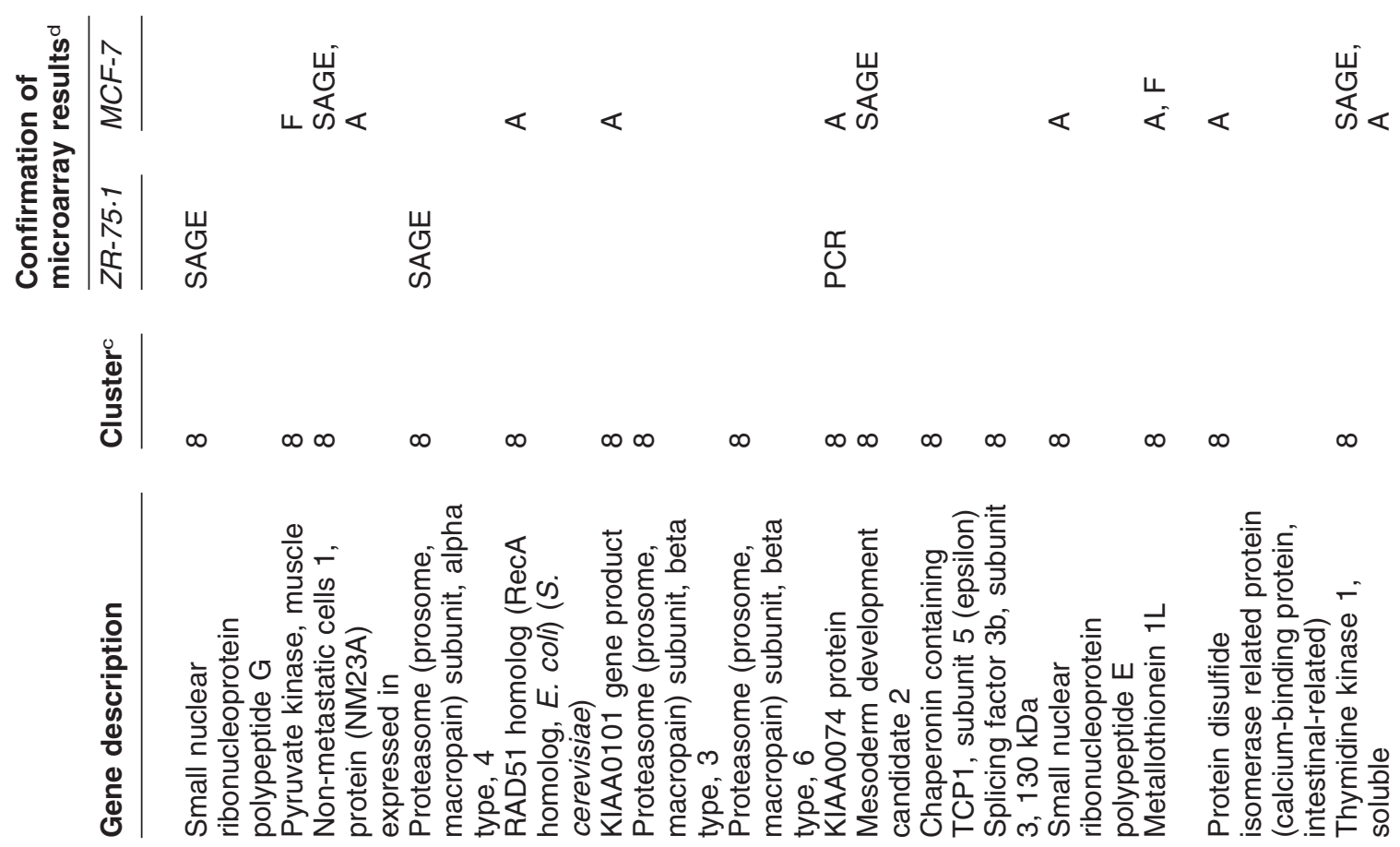

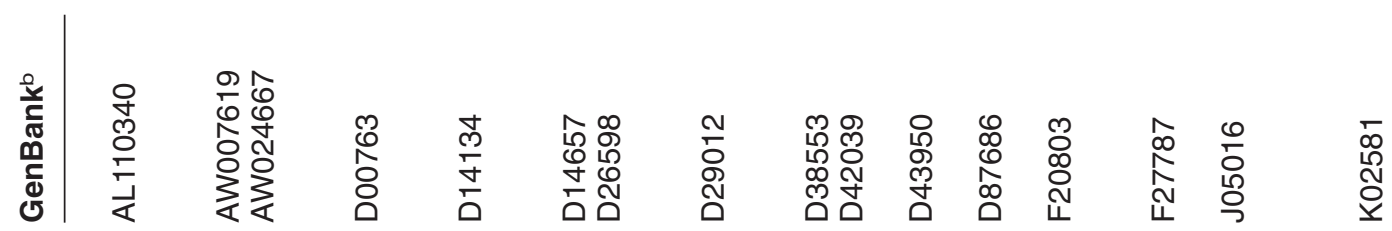

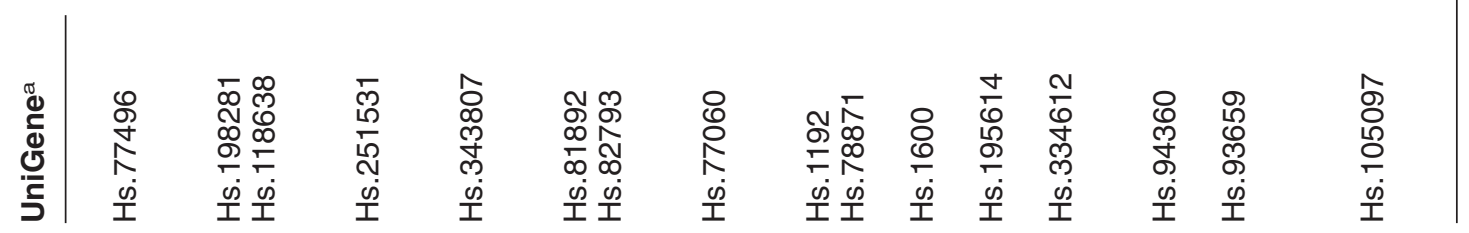

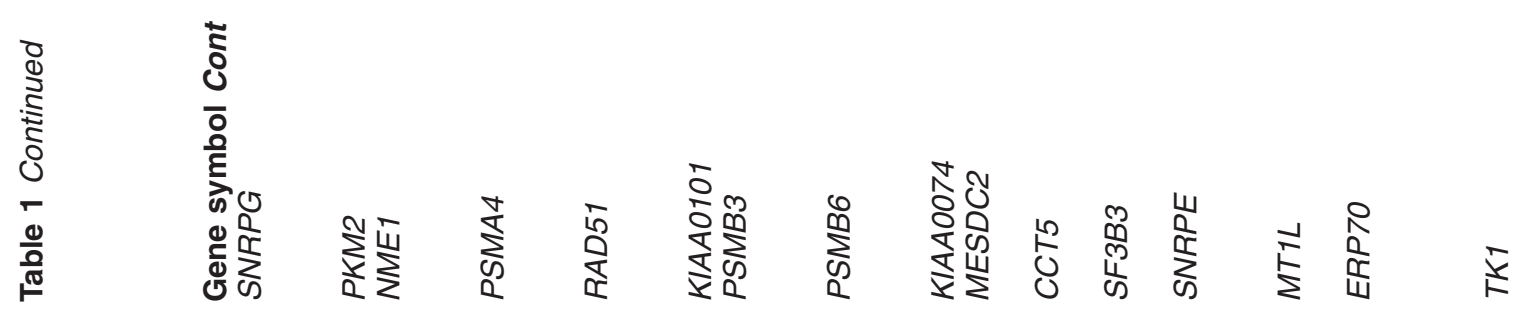



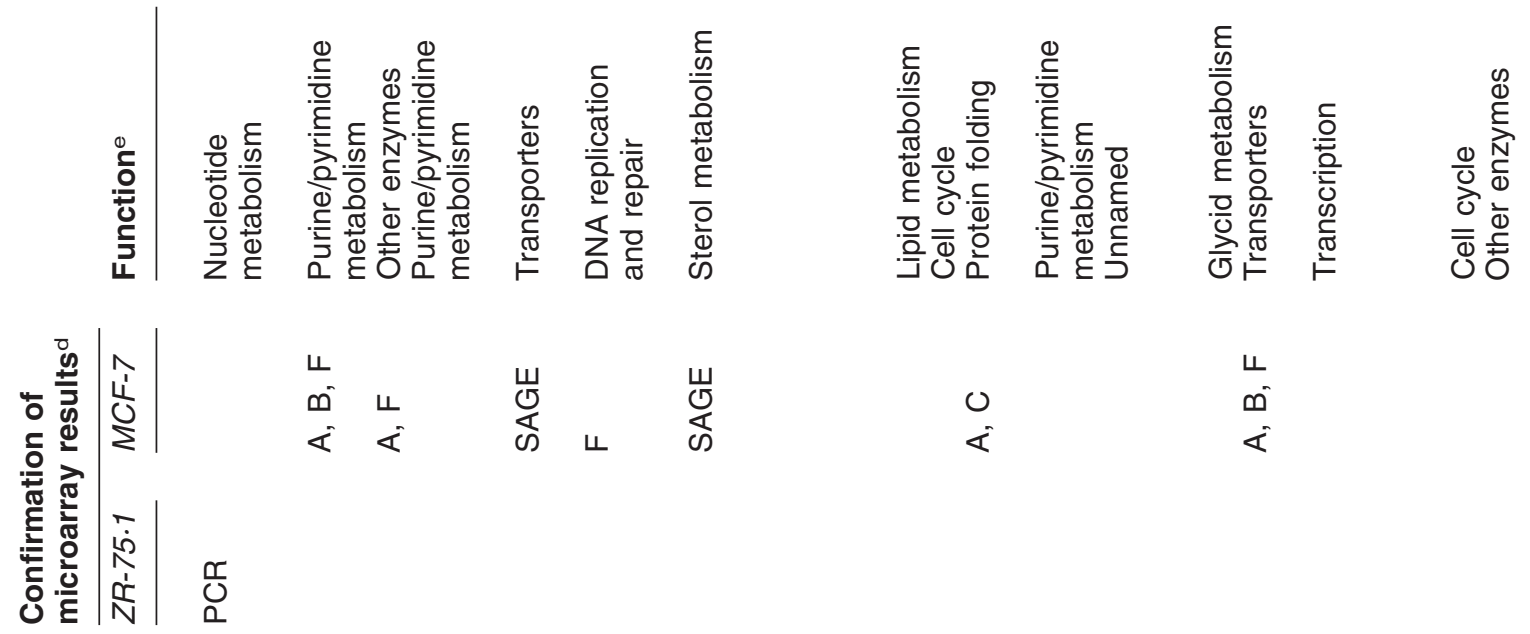
$\begin{array}{ll}\sqcup & \\ \oplus & \longleftarrow \\ \ll & \ll\end{array}$
岕
0
$<$
レ
$\stackrel{0}{i}$

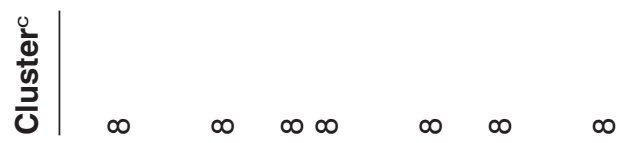

$\infty \infty \infty \quad \infty \quad \infty \quad \infty \infty \quad \infty$

$\infty \infty$

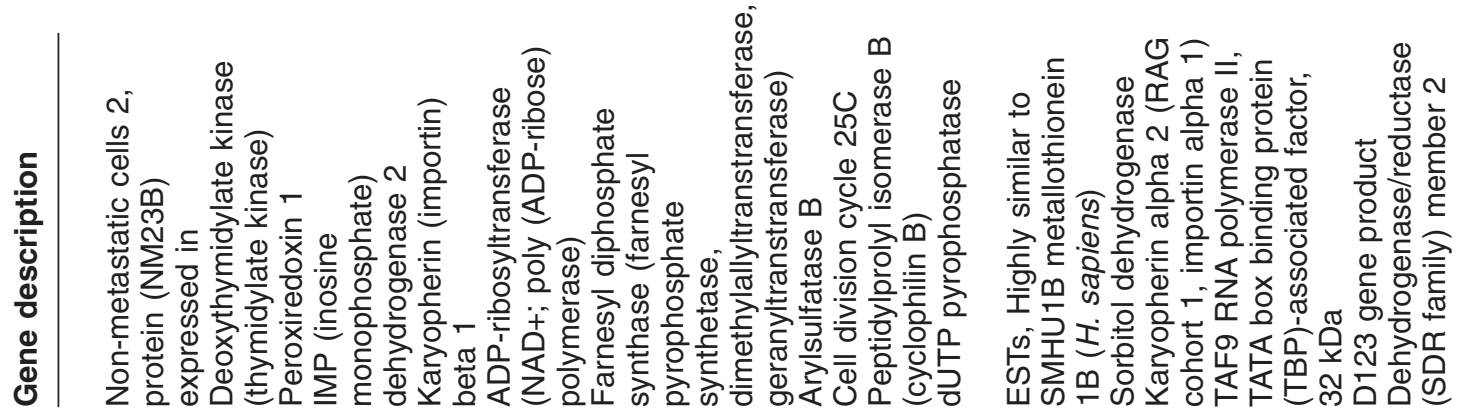

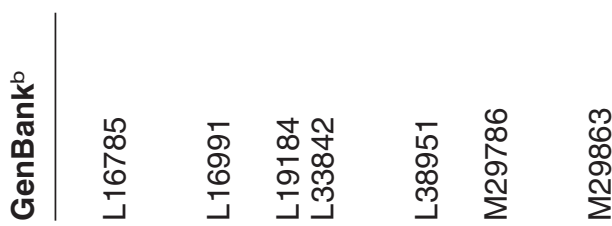

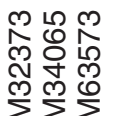

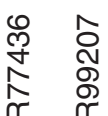

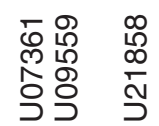

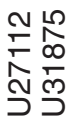

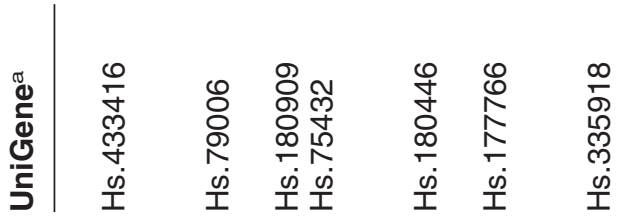

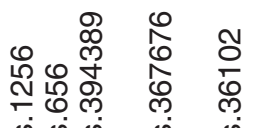

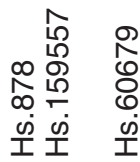

ชุำ

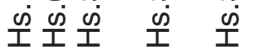

กิ ลิ

오논

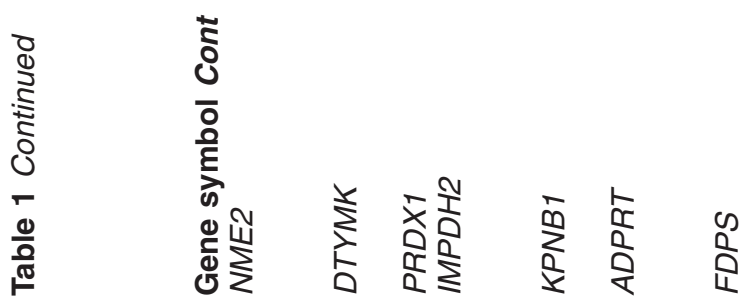

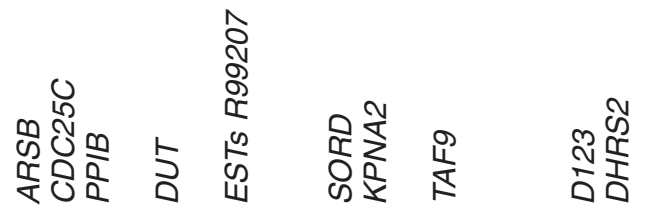




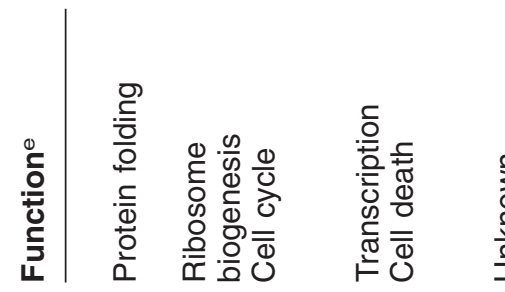
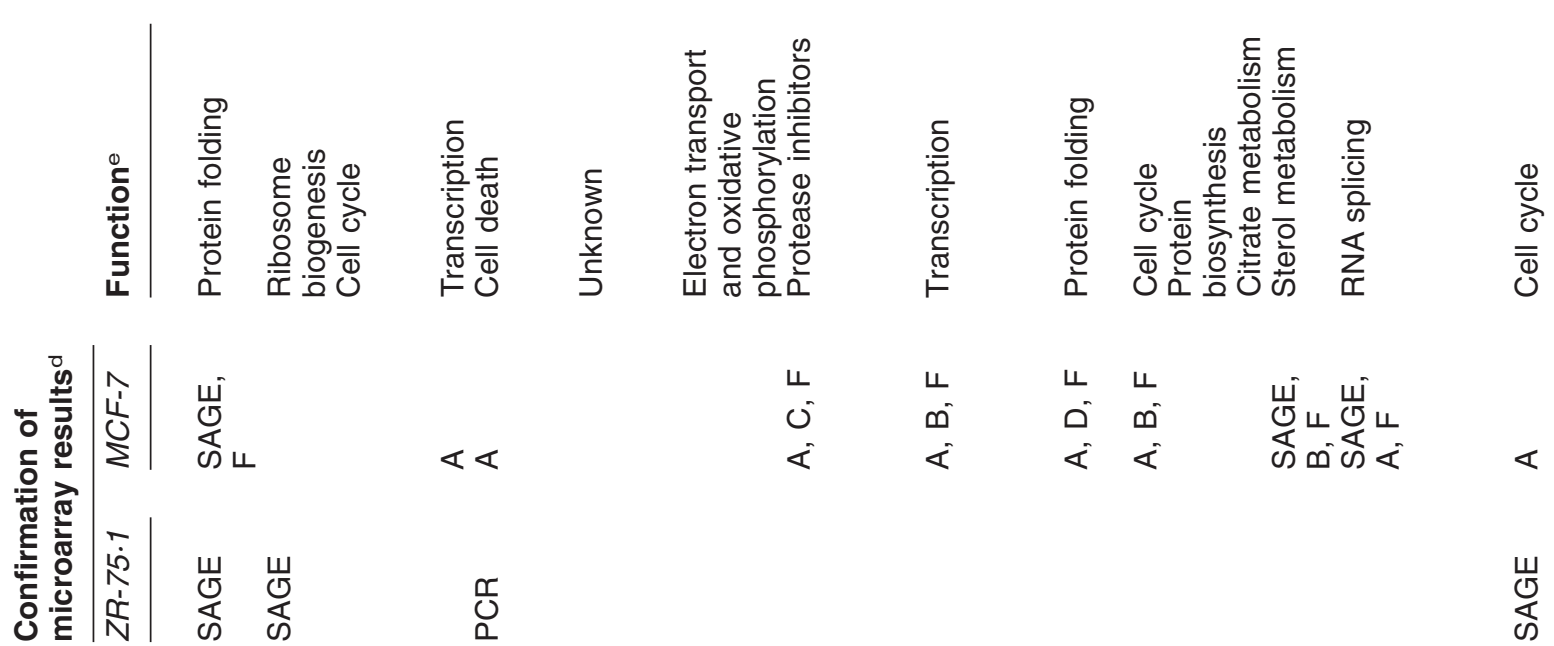

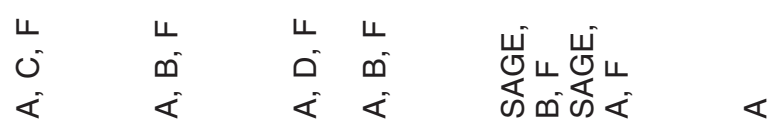

$\stackrel{\Upsilon}{0}$

৫্ড
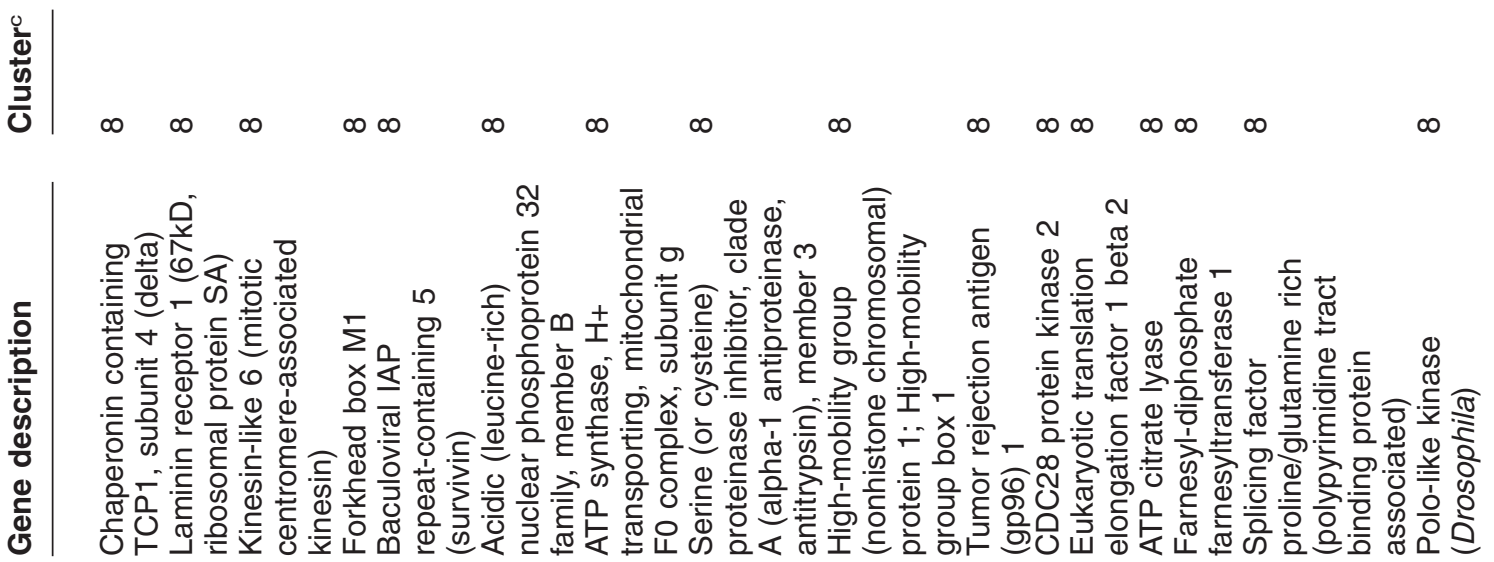

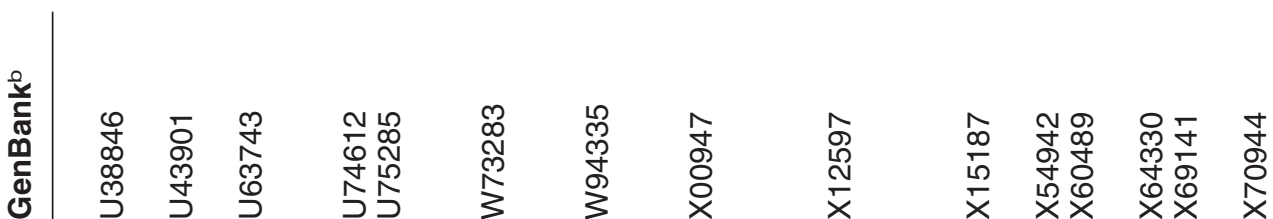

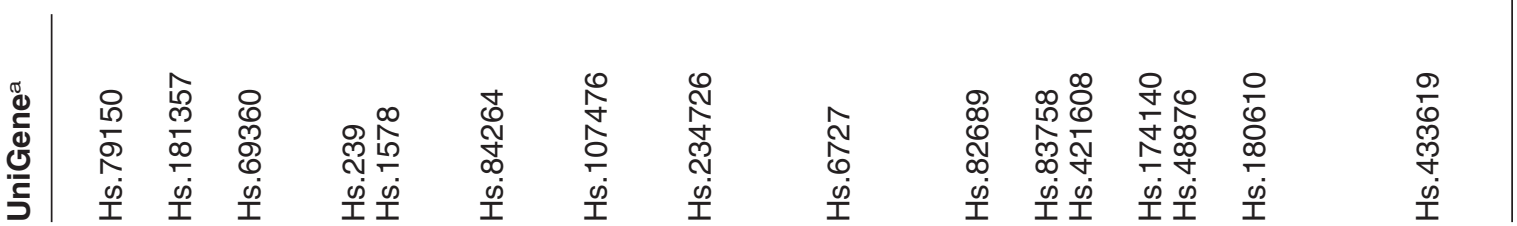

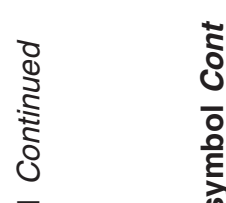

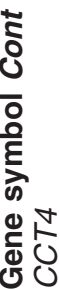

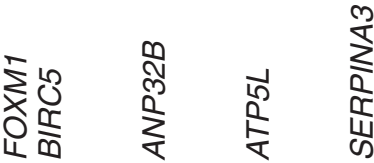

$\sum_{\frac{1}{\infty}}^{m}$

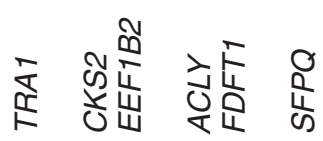

$\frac{x}{2}$ 

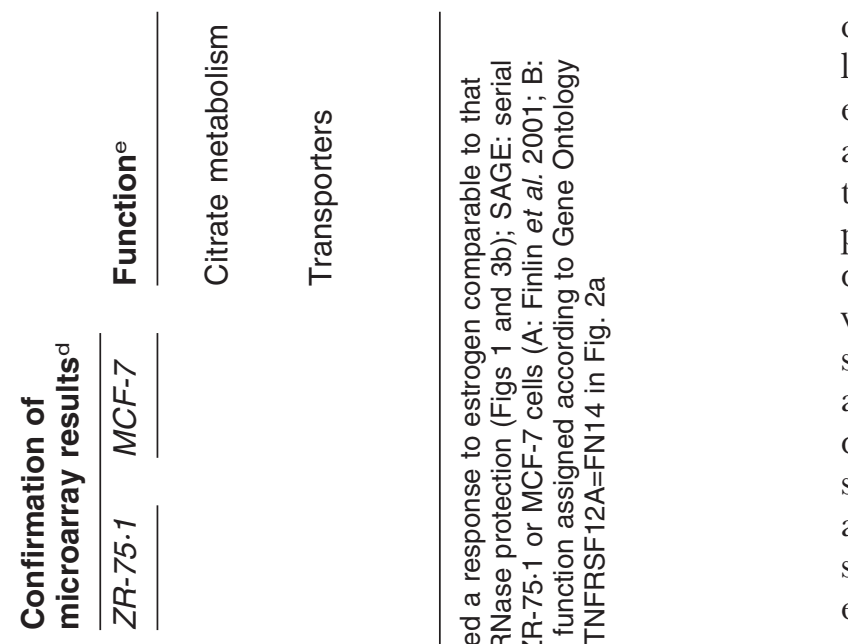

change over multiple consecutive time-points) was lower than that generally applied in global gene expression studies ( $\pm 1 \cdot 8$ - to $2 \cdot 0$-fold change), although within the significance range experimentally determined for the microarray platform and protocols used for this study (Yue et al. 2001). We decided, however, to carry out wherever possible a verification of the gene expression changes summarized in Fig. 2. This was performed according to different strategies, including independent measurements of E2-induced mRNA expression changes in ZR-75.1 cells and functional analyses. Furthermore, we carried out comparative searches for genes showing similar regulation by estrogen in these and other BC cells within publicly available repositories of microarray and SAGE (serial analysis of gene expression) data.

First of all, the expression levels of $19 \mathrm{mRNAs}$ encoded by genes included within the estrogen responsive gene set listed in Table 1 were measured also by fluorigenic quantitative polymerase chain reaction ('real time' rtPGR). The genes to be verified were selected to represent each of the clusters reported in Fig. 2 and to include mRNAs that showed different basal expression and/or magnitude of response to estrogen by microarray analysis. Starting from the sequence of the corresponding cDNA probes in the microarray, specific oligonucleotide primer pairs and probes were designed for each gene, and RNA quantitation was carried out in triplicate both on the same RNA samples used for microarray analysis and on replicate samples prepared thereafter. In this way, we were able to control microarray performance as well as biological reproducibility of the gene responses detected. For one of the genes controlled (interferon alpha-inducible protein, GenBank Acc. no. U22970) no amplification product could be detected, possibly due to wrong selection of the amplification target. For this reason, the microarray results relative to this $\mathrm{mRNA}$ were considered 'not confirmed' and the corresponding gene was eliminated from the set of 345 genes which scored positive during the selection procedures. For 18 other transcripts, PCR products of the expected size were obtained (data not shown) and fluorigenic rtPGR could thus be performed. The results of the corresponding quantitative mRNA assays are reported in Fig. 3a and show that rtPGR confirmed the expression profiles detected by microarray hybridization in all cases investigated. The same

www.endocrinology.org 

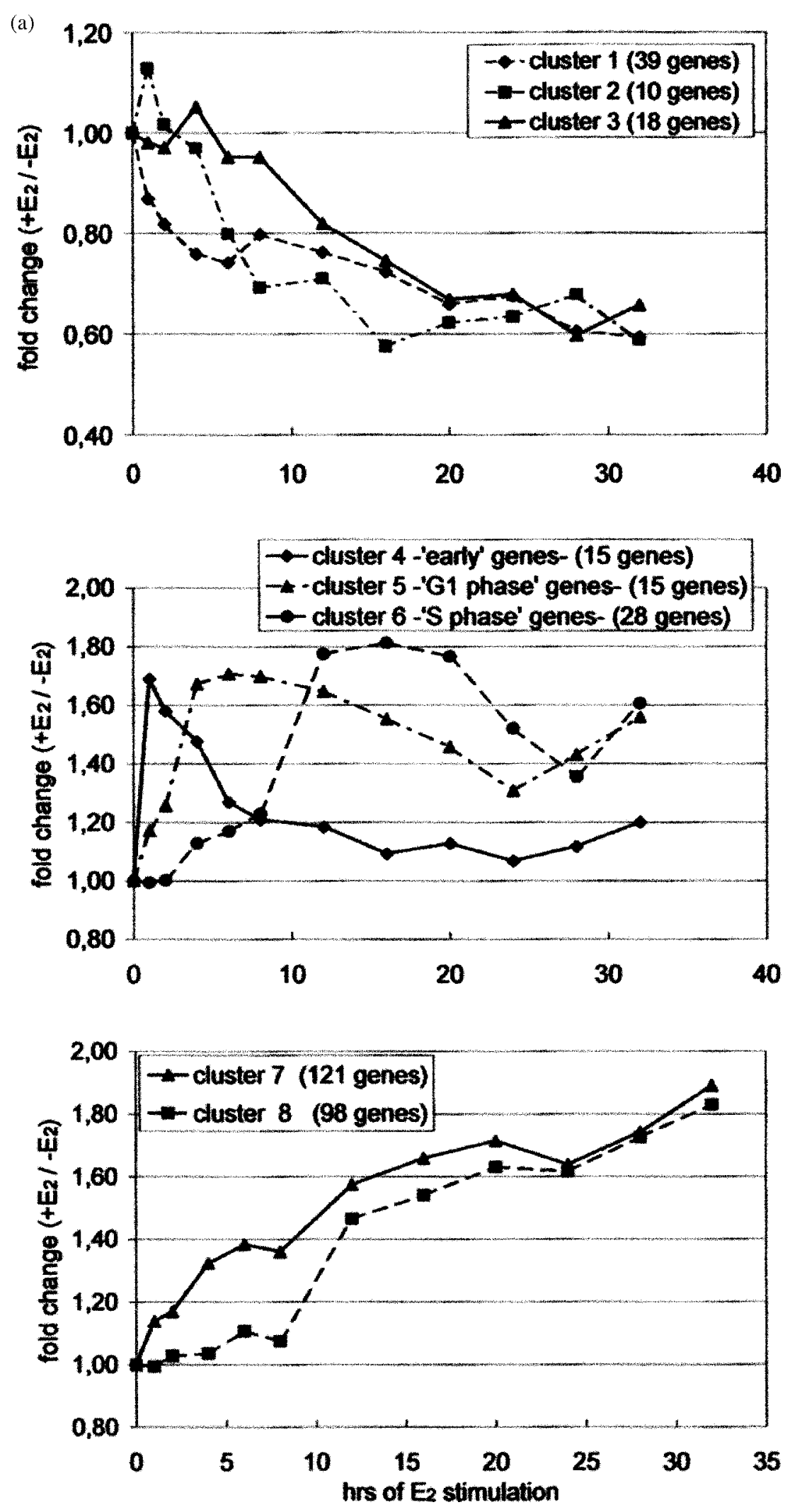
(b)
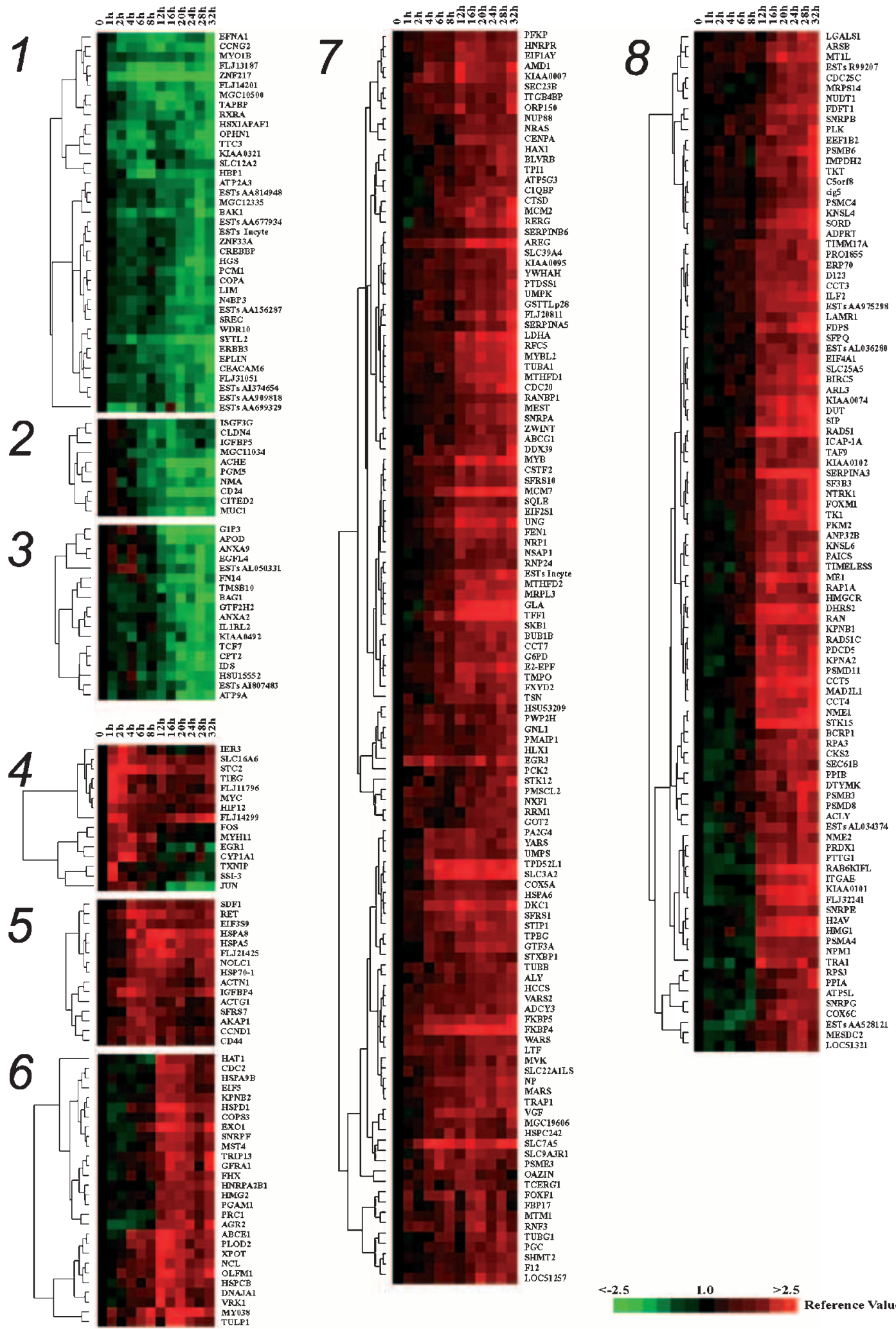
was true for CD44 antigen (homing function and Indian blood group system, GenBank Acc. no. X55150), whose mRNA fluorescent PGR reading was out of scale with respect to all other mRNAs, and for this reason was left out of the figure to optimize its graphical outlook.

A second validation approach consisted in verifying whether the biological information suggested by an increased, or decreased, expression of estrogen-regulated genes reflects known effects of these hormones in $\mathrm{BC}$ cells. To this aim, a functional classification of the genes from Fig. $2 \mathrm{~b}$ was carried out whenever possible, starting from entries in publicly available databases on gene function and is reported in Table 2. Focusing on the 22 genes known to be involved in cell cycle control or progression, all but one of them code for either positive regulators of cell cycle progression or for components of the cell cycle machinery and are activated by the hormone. On the other hand, Cyclin G2, the only gene in this group to be down-regulated by estrogen, encodes a cell cycle inhibitor (Blondel \& Mann 1996, Horne et al. 1997). Furthermore, the timed expression changes observed for these genes in hormone-stimulated cells are in agreement with their pattern of response to mitogens in $\mathrm{BC}$ or other cell types and the known roles of their products in defined cell cycle phases (see also below). This may be considered to represent a 'functional' validation of the microarray results for these genes, as it agrees with the fact that estrogens are potent mitogens, which stimulate cell cycle progression in ZR-75·1 cells (Fig. 1). A second functional group which was investigated in more detail is represented by genes involved in programmed cell death, or apoptosis, which is known to be prevented by estrogen in different cell types, including BC cells where these steroids act as survival factors (Kyprianou et al. 1991). Microarray analysis highlighted 11 genes directly involved in apoptotic processes (Tables 1 and 2). Two of them encode an apoptosis inducer (Bcl2-antagonist/killer1/Bak1) and a pro-apoptotic nuclear receptor (Retinoid $X$ receptor-alpha) and are inhibited by the hormone. On the other hand, 6 genes which are activated by E2 (Heat shock $70 \mathrm{kDa}$ protein 10/HSC71, Heat shock $70 \mathrm{kDa}$ protein 9B/ mortalin 2, Glutathione transferase omega, HS1 binding protein, Immediate early response 3 and Baculovirus IAP repeat-containing $5 /$ survivin) encode well known anti-apoptotic proteins. In the case of the apoptosis-related Bcl2-associated athanogene/Bag1 gene, evaluation of the biological significance of its regulation by estrogen is made difficult by the existence of alternatively spliced mRNA variants in ZR-75.1 cells. These derive from a single transcription unit, give rise to proteins with divergent biological activities (Kudoh et al. 2002), but are indistinguishable by the microarray mRNA analysis carried out here. Hormonal regulation of the Bcl2 partners Bakl and Bagl and of Survivin and $X I A P$ associated factor -1 , belonging respectively to the $B c l 2$ and IAP pathways, suggests that these two functionally related genetic pathways could mediate some of the effects of estrogen on programmed cell death in BC cells. This possibility, suggested by the microarray results, was investigated by determining mRNA expression and hormonal regulation of other, related members of both these gene families in ZR-75.1 cells. The results of mRNA quantitations carried out by multi-probe RPAs are shown in Fig. 3b, where the data relative to $B c l 2$ and its partners are reported on the left, and those related to $I A P$ family genes are reported on the right. The results show that changes in Bakl mRNA expression detected by RPA (Fig. 3b-c), rtPGR (Fig. 3a) and microarrays (Fig. 2b) are consistent. Furthermore, they provide evidence of estrogen-induced inhibition of the pro-apoptotic Bik (Bcl2-inhibitor killer) gene and activation of the anti-apoptotic $B c l 2$, known to be a direct target for transcriptional regulation by ER- $\alpha$ in $\mathrm{MCF}-7$ cells

\footnotetext{
Figure 2 (overleaf) Summary of gene expression changes detected in hormone-stimulated ZR-75.1 cells by cDNA microarray analysis. (a) Graphic representation of the 8 different gene expression clusters identified. The differential expression value (fluorescence reading of labeled cDNA from $+E 2$ cells vs the same from -E2 cells) represents, in each case, the average value for all the genes of the cluster, as detected in multiple measurements. (b) Cluster image showing the 344 mRNAs that showed significant changes of expression in response to estrogen stimulation. The genes were clustered hierarchically into groups (clusters 1 to 8 ) on the basis of the similarity of their expression profiles, as described in the Materials and methods section. The expression pattern of each gene is displayed as a horizontal strip. For each gene, the ratio of mRNA levels in cells at the indicated time after estrogen stimulation vs the same in estrogen-deprived (time 0 ) cells is represented according to the scale reported at the bottom-right of the figure.
} 
Table 2 Functional classification of the estrogen $(E)$ regulated genes identified in ZR-75.1 human breast cancer cells by gene expression profiling with cDNA microarrays according to their predominant functional role and/or to known activities of the products they encode

\section{Number of E-regulated genes}

\section{Functiona}

Signal transduction $\quad 25$

Cell cycle 22

DNA replication and repair 13

Cell death/apoptosis 11

Cell communication, adhesion or motility 19

Gene transcription 31

Messenger RNA processing 12

RNA splicing 6

Ribosome biogenesis $\quad 7$

Protein biosynthesis 10

Protein folding/heat shock response $\quad 16$

Protein degradation $\quad 8$

Transporter 25

Electron transport and oxidative phosphorylation 4

Citrate metabolism 2

Glycid metabolism 10

Lipid metabolism 3

Low-density lipoprotein metabolism 1

Sterol metabolism 5

Xenobiotic metabolism 2

Folate metabolism 2

$\begin{array}{ll}\text { Purine/pyrimidine metabolism } & 10\end{array}$

Enzyme (other than above) 13

Ornithine decarboxylase inhibitor $\quad 1$

Protease inhibitor 2

RNAse L inhibitor $\quad 1$

Heavy metals binding $\quad 1$

Hormone 1

Oxidative stress 1

(Expressed in breast cancer) $^{\mathrm{b}} \quad 2$

$\begin{array}{ll}\text { Not definedc } & 78\end{array}$

aA functional category was assigned to each gene according to Gene Ontology guidelines from LocusLink and/or GeneCards;

'include: 'Trefoil factor 1/pS2' and 'Tumor protein D53-like 1/hD53' genes; cinclude both poorly characterized and unnamed (ESTs) genes.

LDL; ODC,.

(Perillo et al. 2000). Similarly, in the case of the $I A P$ pathway XIAP, cIAP1 and of the IAP-associated tumor necrosis factor receptor-associated factors Traf2 and Traf3 genes are all activated (right image of Fig. 3b). These data, when combined, support the anti-apoptotic effects of the hormone and can thus be considered a further functional confirmation of the indications provided by the microarray results reported in Fig. 2b.

The third validation approach consisted in searching for entries matching genes identified in the present study within publicly available sets of estrogen-regulated genes identified by genomewide expression profiling in hormone-responsive
BC cells. The datasets considered included gene expression data from microarray (Finlin et al. 2001, Bouras et al. 2002, Inoue et al. 2002, Lobenhofer et al. 2002, Hodges et al. 2003) and SAGE (Charpentier et al. 2000, Seth et al. 2002) analyses carried out in E2-stimulated ZR-75.1 or MCF-7 cells. Alignment of microarray and SAGE data obtained in the two cell lines was possible here since they show comparable responses to estrogen in vitro (Cicatiello et al. 2000). Furthermore, similar experimental conditions, including conditions for hormone deprivation and stimulation, were applied in all cases considered. In the case of SAGE data, only mRNAs represented by $\geq 60$ tags, following 
(a)
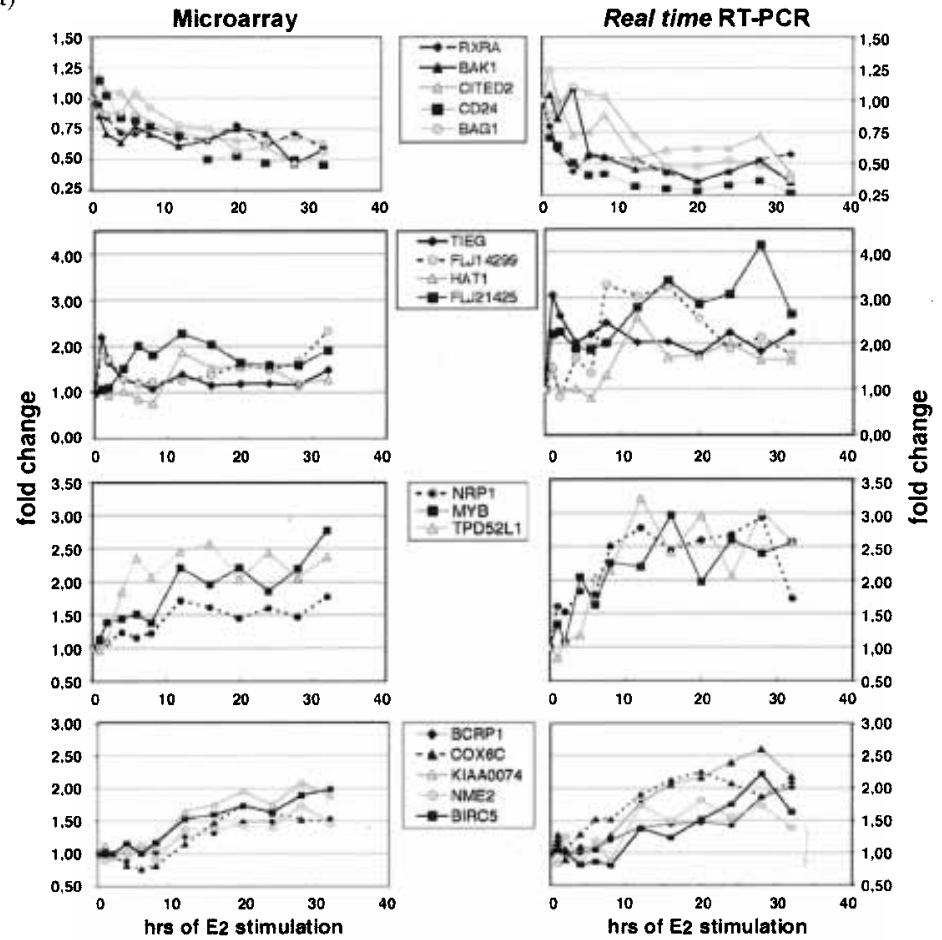

(b)
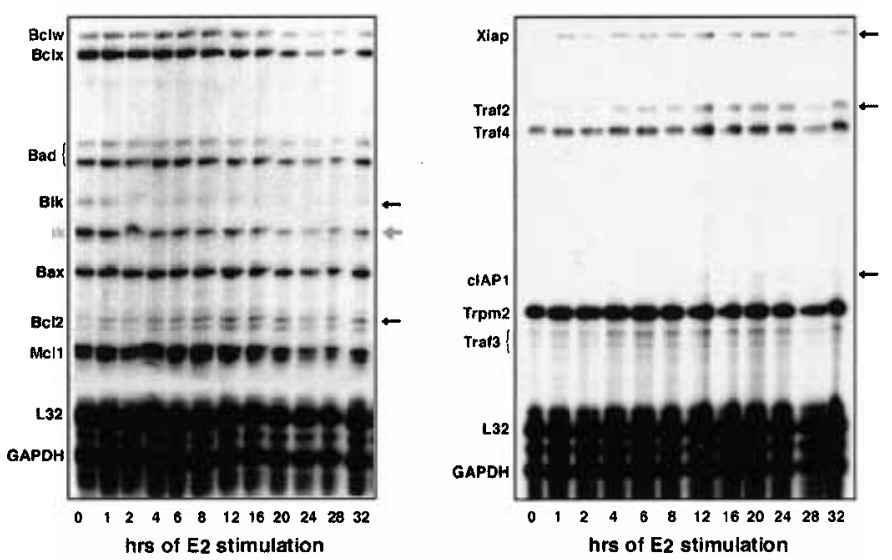

(c)

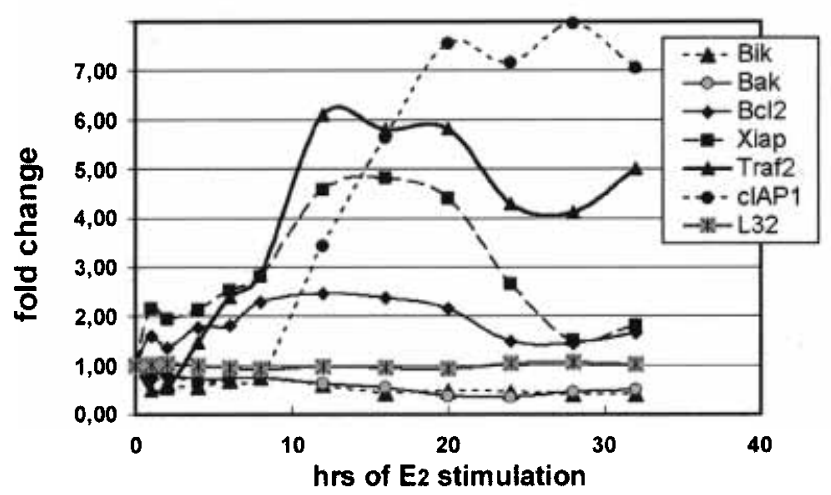


normalization, in either of the matched SAGE libraries were considered for comparison; a transcript scored positive when it showed $\geq$ twofold change in hormone treated vs control cells. The results of these comparative analyses are summarized in the 6th and 7th columns of Table 1. In ZR-75.1 cells, 107 mRNAs from the SAGE libraries were found to match genes from the microarray set of Fig. 2b, including 48 transcripts showing $\geq$ twofold change in expression following cell exposure to the hormone. Concerning the MCF-7 cell SAGE libraries, 116 matches with estrogen-regulated transcripts from Table 1 were found, including 55 mRNAs whose expression changed significantly in response to estrogen also under these conditions. On the other hand, analysis of the microarray data from MCF-7 cells provided confirmation of the expression changes reported in Fig. 2b for 206 genes. These include 76 genes whose hormone responsiveness was confirmed in two or more independent microarray tests and 33 genes which showed a similar response to the hormone in this same cell line also by SAGE (Table 1). When combined, the results of the different tests and comparative analyses described above indicate that estrogen responsiveness appears reproducible for at least 228 genes among those listed in Fig. 2b and Table $1(\sim 2 / 3)$. Hormonal regulation was evident for 148 such genes in two or more experimental settings and, for 45 of them, in two BC cell lines. Considering the latter results, and the fact that direct mRNA measurements performed with 'real time' rtPCR yielded $>94 \%$ confirmations (Fig. 3), we can reasonably assume that the vast majority of the changes in gene expression observed in this study reflect true actions of estrogen on the transcriptome of hormoneresponsive $\mathrm{BC}$ cells.

\section{Comparative analysis of the gene expression changes induced by estrogen and serum growth factors during mitogenic stimulation of human cells}

Given the stimulatory effects of estrogen on cell cycle progression of ZR-75.1 cells, it is likely that some of the gene responses identified in this study are part of the automatic cellular responses of human cells to mitogens. These, occurring in all cells independent of the nature of the stimulus, represent the so called cell cycle 'clock'. To verify this possibility, and to highlight at the same time potential estrogen and/or $\mathrm{BC}$ cell-specific gene responses, the hormone-induced gene expression program was compared with that identified in serum-stimulated human fibroblasts (Iyer et al. 1999). Comparison was made possible since serum stimulation of fibroblasts and estrogen stimulation of ZR-75.1 cells were carried out at comparable time points and the experimental design, including the cDNA microarray platform, was similar. Data were compared for identical time points, from 0 to $24 \mathrm{~h}$ of stimulation in each case, and genes were matched in the two databases by name, UniGene cluster and GB Accession number. One hundred and forty-eight estrogen-regulated genes were found in the serum-stimulated fibroblasts database and their expression profiles were compared in the two stimulated cell types by calculating, in each case, the Pearson's correlation coefficient, accompanied by manual inspection of the data for further confirmation. Fifty-two such genes displayed similar expression profiles in both cell lines (Pearson's $>0 \cdot 40$; Table 3), suggesting that they are likely to be part of a cell type-independent genomic response to extracellular stimuli and/or that, at least in some cases, they reflect similarities

Figure 3 Experimental validation of the microarray results. (a) Real time, fluorigenic quantitative rtPCR assays were carried out as described in the Materials and methods section. Average PCR data were plotted in each case relative to the values detected in RNA from estrogen-deprived cells (time 0). Genes are grouped according to clusters (from top: clusters 1 to 3 , clusters 4 to 6 , cluster 7 and cluster 8 ) and microarray results (left) are reported side-by-side with the corresponding rtPCR results (right), to allow direct comparisons. (b) RPAs (RNase protection assays) were carried out in the same RNA samples as described in the Materials and methods section. For details concerning gene names, see the text. L32 and GAPDH are internal controls, as reported in the legend to Fig. 1. Bars mark regulated genes, the gray bar indicates that BAK1 mRNA showed comparable changes by cDNA microarray analysis. (c) Graphic representation of data from apoptosis-related gene expression monitoring by RPA. Average of results from three independent measurements are reported only for regulated mRNAs and for L32 control mRNA, following normalization with respect to GAPDH mRNA levels in each lane. 

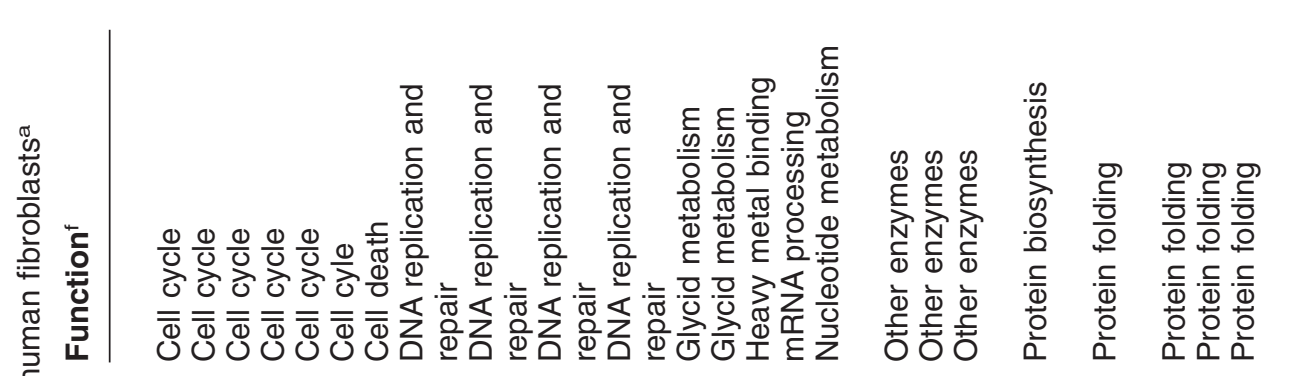

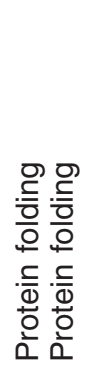

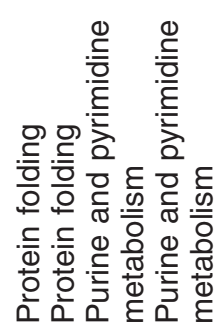

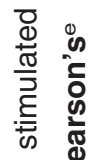

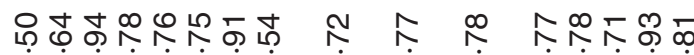

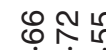

西

ก

实

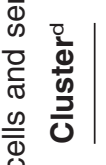

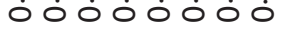
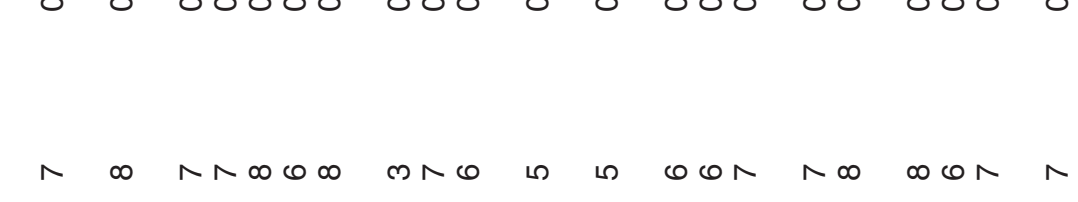

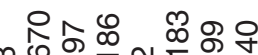

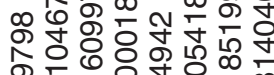

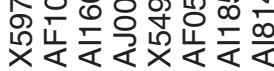

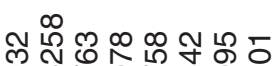

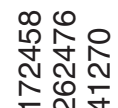

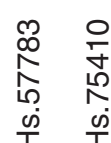

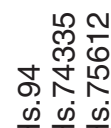

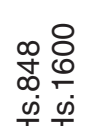

象总

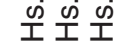

오오오

禹定

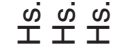



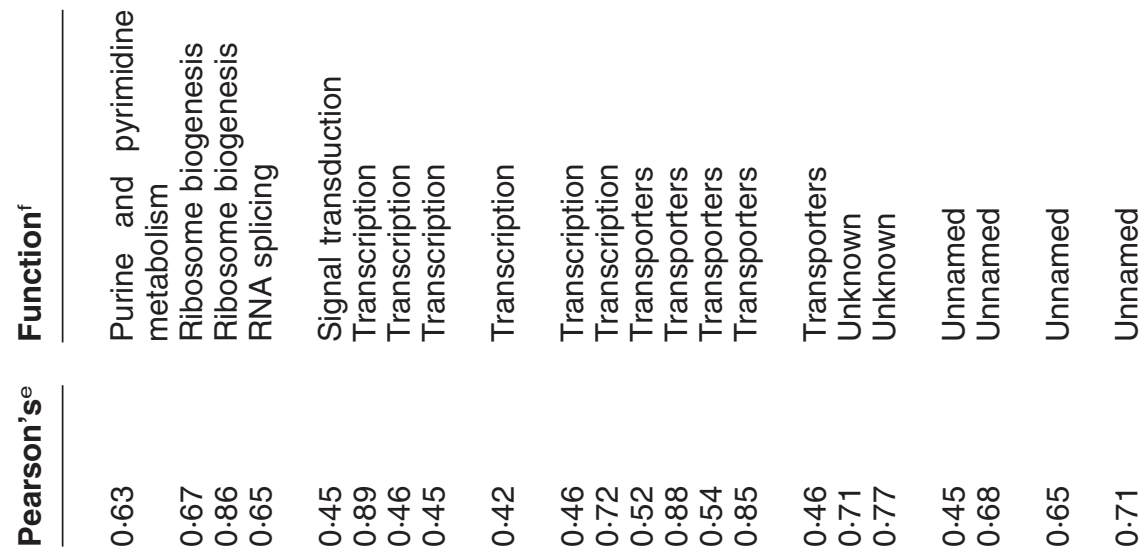

$\frac{}{\frac{1}{d}}$
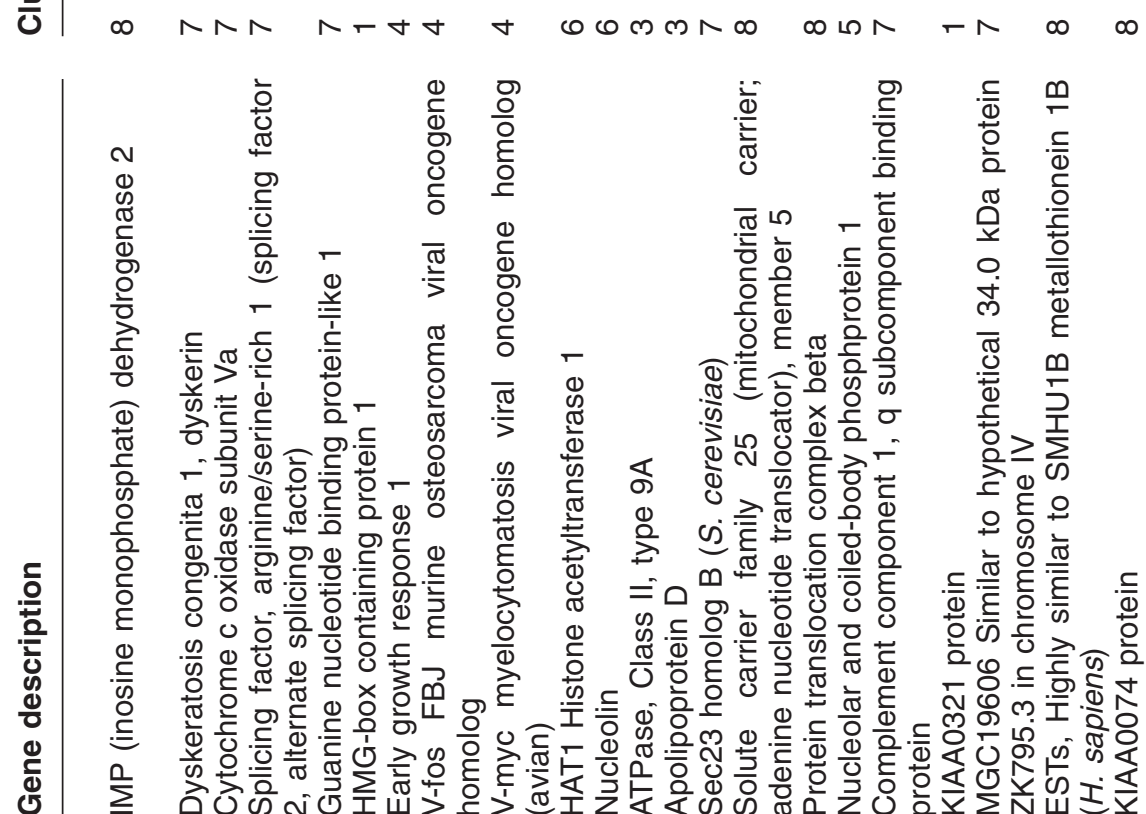

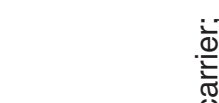

$\frac{\sqrt{\frac{\pi}{2}}}{\frac{2}{2}}$

잉

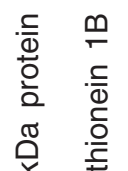

는

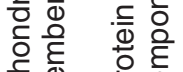

잉 ๘

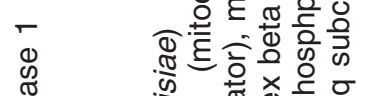

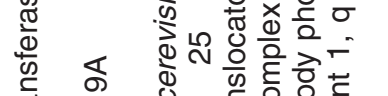

స

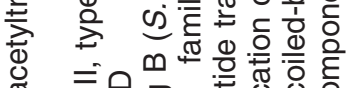

ช

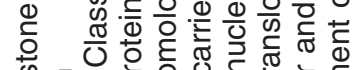

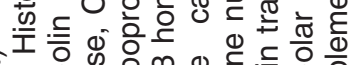

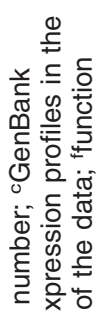

㐫

응

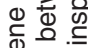

등

$\frac{\pi}{0} \cdot \frac{0}{>}$

हे 할

근

क ष

융 흘

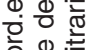

은

苗.

은 원

3.

듀류

인은 웅

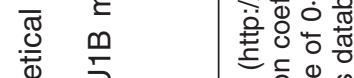

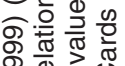

은 응

ำ $\geq \sum_{0}$

으웡 웅

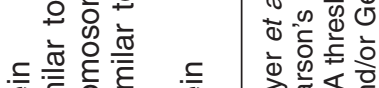

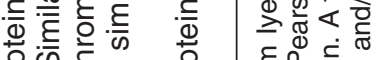

응 向》の

으드음

సั

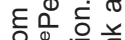

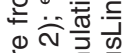

बं $\dot{0}$.

उ谣守

上过

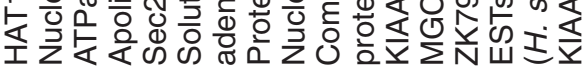

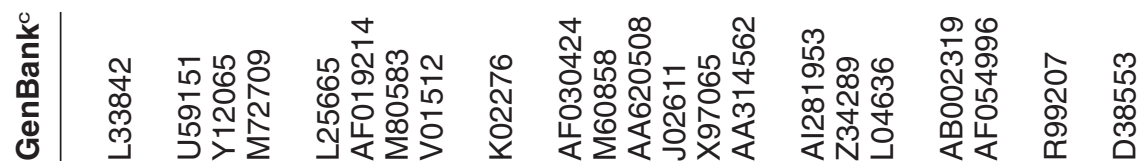

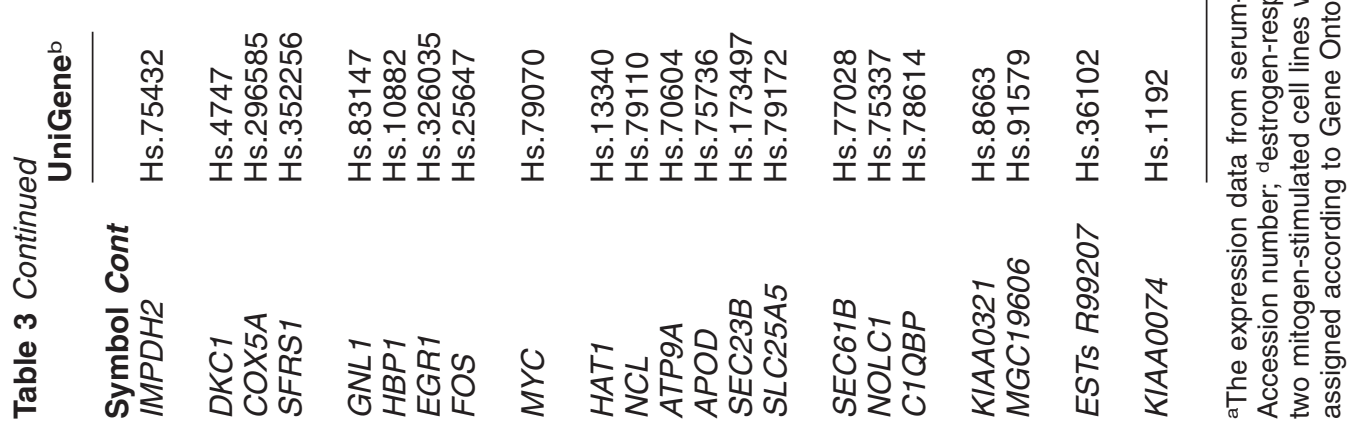


between signaling by serum factors and estrogen. This last possibility is suggested by a number of reports indicating 'non-genomic' actions of estrogen in target cells leading to direct hormonal regulation of multiple cytoplasmic signal transduction pathways (reviewed by Kelly \& Levin 2001). In agreement with this last possibility, the 'immediateearly' EGR1 (early growth response 1) gene, which is among the genes showing comparable changes in BC cells and fibroblasts (Table 3), has been reported to be activated by estrogens in cardiomyocytes via serum-responsive DNA elements present in its promoter (de Jager et al. 2001). On the other hand, genome-scale gene expression analysis in growth factor-stimulated cells has revealed that diverse signaling pathways activated by different mitogen receptors induce broadly overlapping, rather than independent, sets of genes (Fambrough et al. 1999). This suggests that individual signal transduction pathways evoke in a given cell redundant patterns of gene expression, which are independent from the nature of the signaling pathway involved (Pawson \& Saxton 1999). Indeed, some of the genes co-regulated by estrogens and serum mitogens (Table 3) belong to the recently identified transcriptional program of the cell division cycle of human cancer cells, which includes genes undergoing automatic, periodic changes in expression linked to specific cell cycle phases (see below). On the other hand, eighteen genes among those common to the two datasets compared show opposite changes in BC vs fibroblasts (Pearson's $<-0 \cdot 40$; Table 4), while the remaining seventy eight estrogen targets are unresponsive to serum in fibroblasts (Table 5). These two gene sets are more likely to include hormone and/or BC-specific genomic targets, used to understand the nature of specific estrogen effects in BC cells.

\section{Identification of the estrogen-regulated genes which belong to the transcriptional program of the cell division cycle}

Assignment of genes to known genetic programs, such as, for example, cell regulatory circuits and metabolic or functional pathways, may greatly help the functional interpretation of genome-wide gene expression profiling data (Ashburner et al. 2000). In the case of the gene expression changes induced by estrogen this analysis is particularly important, since it may help provide logical frameworks to highlight key aspects of hormone-responsive $\mathrm{BC}$ cell biology and to elucidate the mechanism of action of hormones and antihormones.

As an initial step in this direction, each gene was assigned, where possible, to a functional category according to the Gene Ontology Consortium guidelines (last column of Table 1). One of the observations made possible by this analysis is that a significant number of estrogenregulated genes appear to relate to functions associated with the cell division cycle. This led us to pursue our quest for gene expression changes which may represent predominantly cell cycle responses to the mitogenic stimulus exerted by $\mathrm{E} 2$, and for this reason are less likely to include estrogen- or BC-specific gene responses. With this in mind, we searched within our list of estrogen-responsive genes for members of the recently identified cell cycle transcriptional program of human cells (Whitfield et al. 2002). This analysis yielded the 51 estrogen-responsive genes listed in Table 6, which are among those characterized by periodic expression during the mitotic cycle in synchronized cells. For twentyfour such genes, all activated by the hormone, the inferred function supports their inclusion in this category. Indeed, sixteen of them encode proteins functionally involved in cell cycle phase control/ accomplishment, seven are involved in DNA replication and repair and one in nucleotide metabolism, while two encode for tubulins (alpha 1 and beta). The functions of six other genes are unknown, while those of the remaining nineteen, suggesting their involvement in gene transcription, mRNA processing, splicing and transport, ribosome biogenesis, protein folding and degradation or metabolism, are compatible with a role of these gene products during cell cycle progression. It is worth mentioning that ten genes among those listed in Table 6 (i.e. CDC28 protein kinase 2/CKS2, Cyclin D1/PRAD1, Dyskeratosis congenita 1-dyskerin/ DKC1, Flap structure-specific endonuclease 1/FEN1, Member of the RAS oncogene family/RAN, Minichromosome maintenance deficient 2-mitotin/MCM2, Mitotic arrest deficient-like 1/MAD2 L1, Ribonucleotide reductase M1 polypeptide/RRM1, S-adenosylmethionine decarboxylase 1/AMD1 and KLAA0074 protein) show comparable expression patterns in estrogenstimulated ZR-75.1 cells and serum-stimulated fibroblasts (see Table 3). This finding supports the view that regulation of these genes in estrogen-stimulated cells is part of the anabolic 


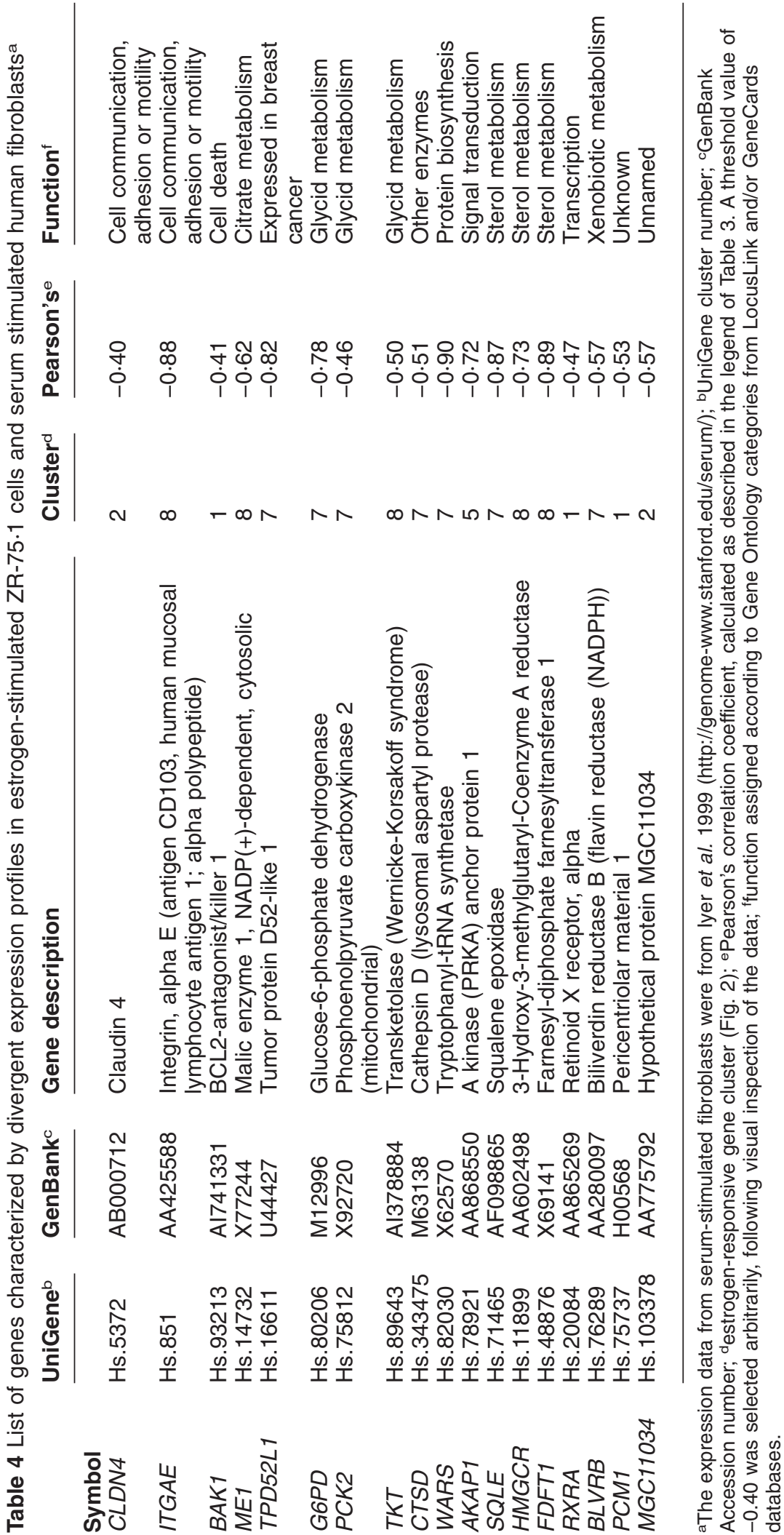


Table 5 List of estrogen-regulated genes which were found unresponsive to serum stimulation in human fibroblasts by cDNA microarray gene expression analysisa

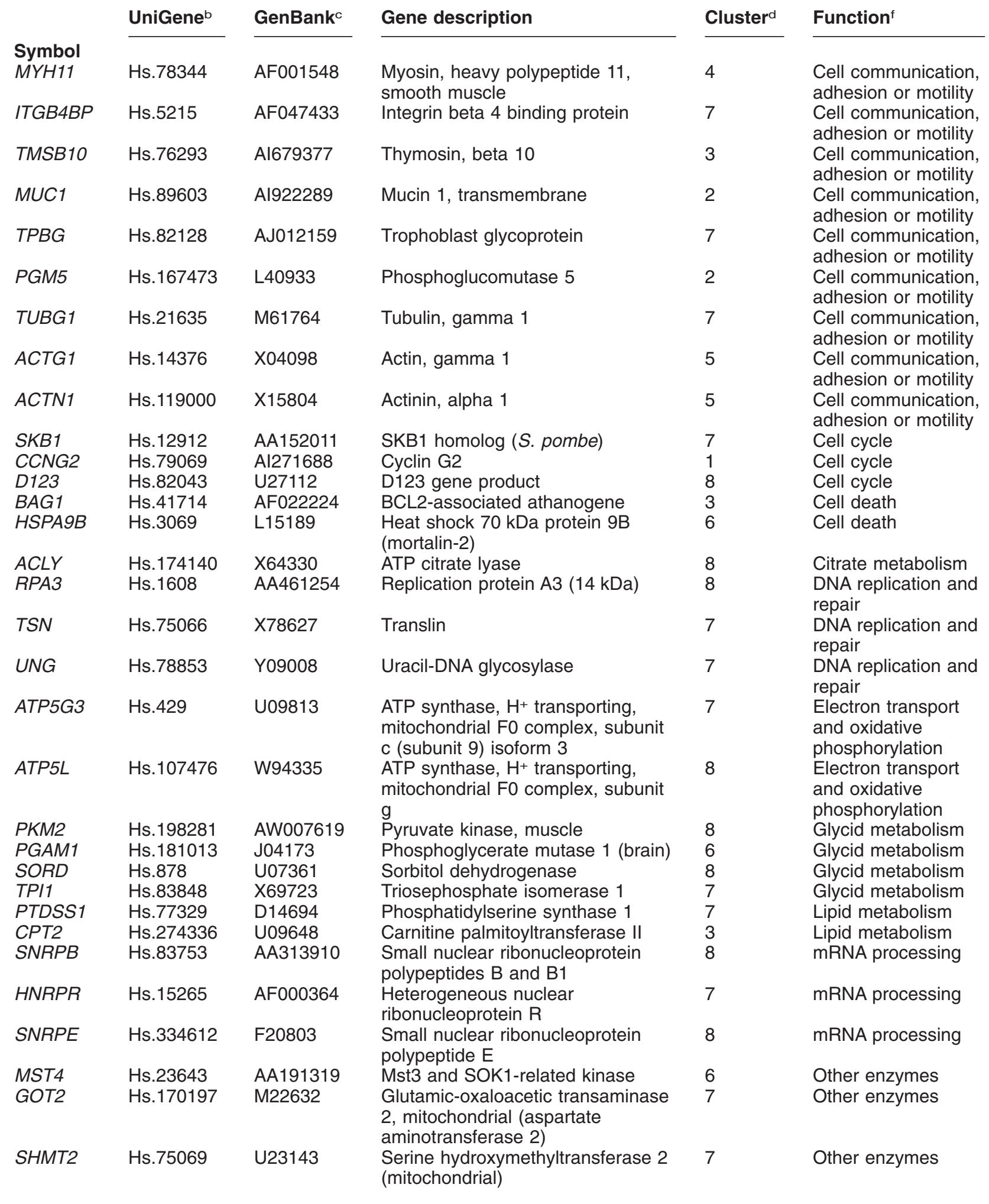


Table 5 Continued

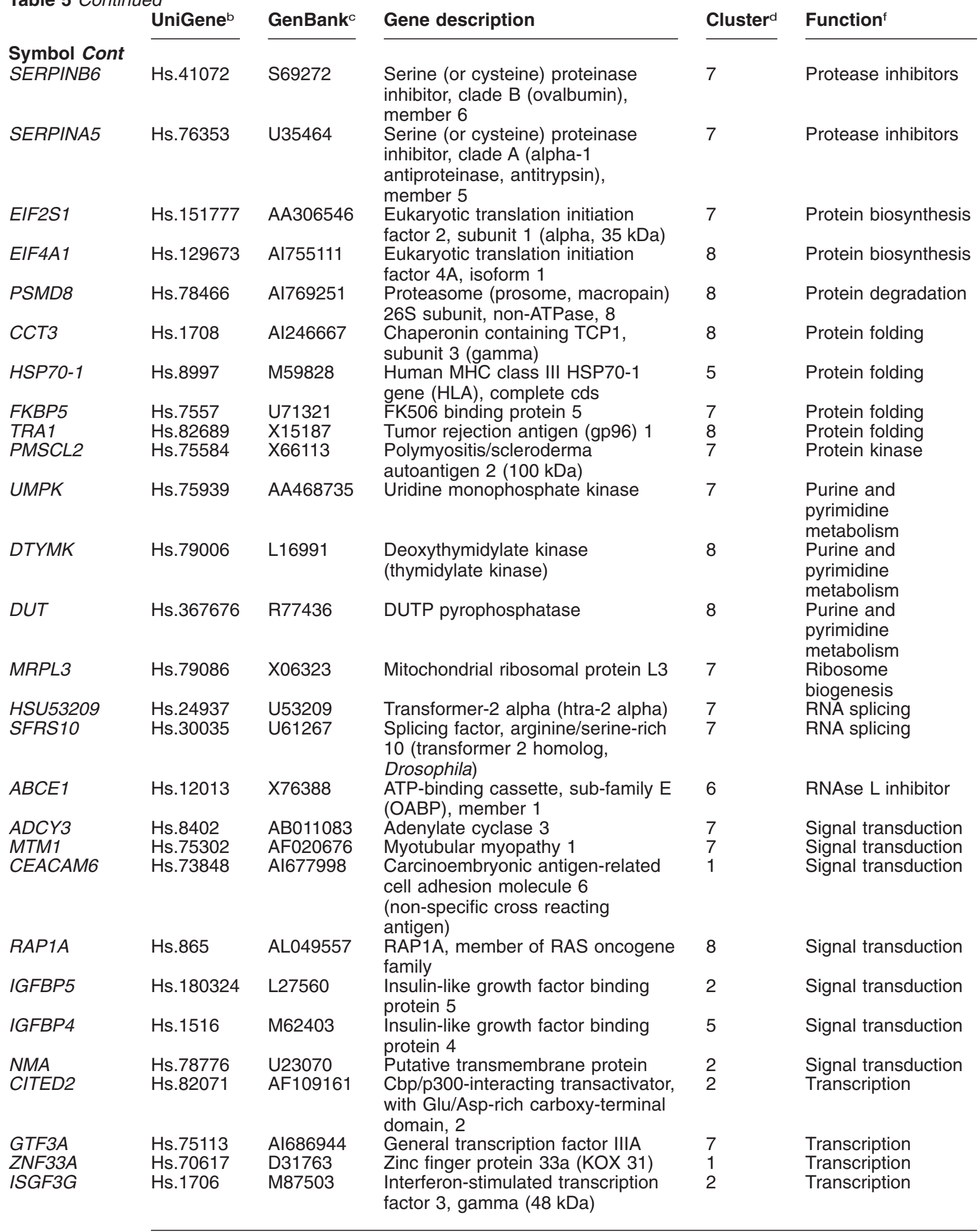


Table 5 Continued

\begin{tabular}{|c|c|c|c|c|c|}
\hline & UniGene $^{b}$ & GenBank $^{\mathrm{C}}$ & Gene description & Cluster ${ }^{d}$ & Function $^{f}$ \\
\hline $\begin{array}{l}\text { Symbol Cont } \\
M Y B\end{array}$ & Hs.1334 & U22376 & $\begin{array}{l}\text { V-myb myeloblastosis viral } \\
\text { oncogene homolog (avian) }\end{array}$ & 7 & Transcription \\
\hline SLC3A2 & Hs.79748 & AB018010 & $\begin{array}{l}\text { Solute carrier family } 3 \text { (activators of } \\
\text { dibasic and neutral amino acid } \\
\text { transport), member } 2\end{array}$ & 7 & Transporters \\
\hline$A B C G 1$ & Hs.10237 & AF038175 & $\begin{array}{l}\text { ATP-binding cassette, sub-family G } \\
\text { (WHITE), member } 1\end{array}$ & 7 & Transporters \\
\hline LTF & Hs.105938 & M73700 & Lactotransferrin & 7 & Transporters \\
\hline COPA & Hs.75887 & U24105 & $\begin{array}{l}\text { Coatomer protein complex, subunit } \\
\text { alpha }\end{array}$ & 1 & Transporters \\
\hline SLC12A2 & Hs.110736 & U30246 & $\begin{array}{l}\text { Solute carrier family } 12 \\
\text { (sodium/potassium/chloride } \\
\text { transporters), member } 2\end{array}$ & 1 & Transporters \\
\hline ATP2A3 & Hs.5541 & Z69881 & $\begin{array}{l}\text { ATPase, } \mathrm{Ca}^{++} \text {transporting, } \\
\text { ubiquitous }\end{array}$ & 1 & Transporters \\
\hline RNF3 & Hs.8834 & AA403225 & Ring finger protein 3 & 7 & Unknown \\
\hline $\mathrm{PWP2H}$ & Hs.79380 & AB001517 & $\begin{array}{l}\text { PWP2 periodic tryptophan protein } \\
\text { homolog (yeast) }\end{array}$ & 7 & Unknown \\
\hline MESDC2 & Hs.78871 & D42039 & $\begin{array}{l}\text { Mesoderm development candidate } \\
2\end{array}$ & 8 & Unknown \\
\hline PMAIP1 & Hs.96 & D90070 & $\begin{array}{l}\text { Phorbol-12-myristate-13-acetate- } \\
\text { induced protein } 1\end{array}$ & 7 & Unknown \\
\hline HSU15552 & Hs.85769 & U15552 & Acidic $82 \mathrm{kDa}$ protein mRNA & 3 & Unknown \\
\hline G1P3 & Hs.265827 & U22970 & $\begin{array}{l}\text { Interferon, alpha-inducible protein } \\
\text { (clone IFI-6-16) }\end{array}$ & 3 & Unknown \\
\hline FLJ20811 & Hs.83530 & AA015605 & FLJ20811 Hypothetical protein & 7 & Unnamed \\
\hline FLJ13187 & Hs.29724 & AA713516 & FLJ13187 Phafin 2 & 1 & Unnamed \\
\hline C5orf8 & Hs.75864 & Al954986 & $\begin{array}{l}\text { Chromosome } 5 \text { open reading frame } \\
8\end{array}$ & 8 & Unnamed \\
\hline KIAA0102 & Hs.77665 & AL047241 & KIAA0102 gene product & 8 & Unnamed \\
\hline KIAA0007 & Hs.90315 & D26488 & KIAA0007 protein & 7 & Unnamed \\
\hline
\end{tabular}

aThe expression data from serum-stimulated fibroblasts were from lyer et al. (1999) (http://genome-www.stanford.edu/serum/). Differential expression data were compared between the two experimental data sets by calculating the Pearson's correlation coefficient value for the data relative to the first $24 \mathrm{~h}$ of stimulation, as described in the legend of Table 3 . The data were then further controlled by visual inspection; bUniGene cluster number; ' GenBank Accession number; destrogen-responsive gene cluster (Fig. 2); efunction assigned according to Gene Ontology categories from LocusLink and/or GeneCards databases.

effect of these steroids on the cell cycle machinery.

In conclusion, the results of all these comparative analyses indicate that the gene expression program activated by estrogen in BC cells reflects more than a simple mitogenic response of the cell to the hormone, since as many as 293 among its genes do not appear to belong to the transcriptional program of the cell division cycle.

\section{Analysis of the distribution of estrogen-regulated genes among chromosomes}

Genome-wide analyses of human transcriptome maps consistently indicate that the genes expressed in each given cell type often cluster in defined chromosomal regions, which have been called RIDGEs (regions of increased gene expression; Caron et al. 2001). Genes expressed in skeletal muscle, for example, have been found selectively concentrated in certain human chromosomes (Bortoluzzi et al. 1998). Similar evidence of a gene order in the human genome has also been provided by analysis of housekeeping genes (Lercher et al. 2002), which suggests how the expression neighborhood may serve to regulate the functions of these genes (Oliver et al. 2002). Similarly, Spellman and Rubin (2002) observed in Drosophila that neighboring genes are often expressed in similar patterns. Such a phenomenon is not limited to the genomes 
Table 6 List of genes regulated by estrogen in ZR-75.1 cells which are characterized by periodic expression during the cell cycle in human cells

\begin{tabular}{|c|c|c|c|c|c|}
\hline & Symbol & UniGene $^{c}$ & Gene description & Cluster ${ }^{d}$ & Functione \\
\hline $\begin{array}{l}\text { Phase }^{\mathrm{b}} \\
\text { M/G1 }\end{array}$ & CCND1 & Hs.82932 & $\begin{array}{l}\text { Cyclin D1 (PRAD1: parathyroid } \\
\text { adenomatosis 1) }\end{array}$ & 5 & Cell cycle \\
\hline M/G1 & PRC1 & Hs.344037 & Protein regulator of cytokinesis 1 & 6 & Cell cycle \\
\hline $\mathrm{M} / \mathrm{G} 1$ & $R A N$ & Hs. 10842 & $\begin{array}{l}\text { RAN, member RAS oncogene } \\
\text { family }\end{array}$ & 8 & Cell cycle \\
\hline M/G1 & NSAP1 & Hs.155489 & NS1-associated protein 1 & 7 & mRNA processing \\
\hline $\mathrm{M} / \mathrm{G} 1$ & $A M D 1$ & Hs.262476 & $\begin{array}{l}\text { S-adenosylmethionine } \\
\text { decarboxylase } 1\end{array}$ & 7 & Other enzymes \\
\hline $\mathrm{M} / \mathrm{G} 1$ & $E 2-E P F$ & Hs. 174070 & Ubiquitin carrier protein & 7 & Protein degradation \\
\hline M/G1 & HSPA8 & Hs.180414 & Heat shock 70 kDa protein 8 & 5 & Protein folding \\
\hline M/G1 & $D K C 1$ & Hs.4747 & Dyskeratosis congenita 1 , dyskerin & 7 & Ribosome biogenesis \\
\hline $\mathrm{M} / \mathrm{G} 1$ & PTTG1 & Hs.252587 & Pituitary tumor-transforming 1 & 8 & Signal transduction \\
\hline $\mathrm{M} / \mathrm{G} 1$ & HMGCR & Hs.11899 & $\begin{array}{l}\text { 3-Hydroxy-3-methylglutaryl-Coenzyme } \\
\text { A reductase }\end{array}$ & 8 & Sterol metabolism \\
\hline M/G1 & ILF2 & Hs.75117 & $\begin{array}{l}\text { Interleukin enhancer binding factor } \\
2,45 \mathrm{kDa}\end{array}$ & 8 & Transcription \\
\hline M/G1 & NXF1 & Hs.323502 & Nuclear RNA export factor 1 & 7 & Transporters \\
\hline M/G1 & KPNB1 & Hs.180446 & Karyopherin (importin) beta 1 & 8 & Transporters \\
\hline $\mathrm{G} 1 / \mathrm{S}$ & MCM2 & Hs.57101 & $\begin{array}{l}\text { MCM2 minichromosome } \\
\text { maintenance deficient 2, mitotin ( } S \text {. } \\
\text { cerevisiae) }\end{array}$ & 7 & $\begin{array}{l}\text { DNA replication and } \\
\text { repair }\end{array}$ \\
\hline G1/S & UNG & Hs.78853 & Uracil-DNÁ glycosylase & 7 & $\begin{array}{l}\text { DNA replication and } \\
\text { repair }\end{array}$ \\
\hline G1/S & SFRS7 & Hs.184167 & $\begin{array}{l}\text { Homo sapiens splicing factor, } \\
\text { arginine/serine-rich } 7 \text { (SFRS7) } \\
\text { gene, complete cds }\end{array}$ & 5 & RNA splicing \\
\hline G1/S & HSU53209 & Hs.24937 & Transformer-2 alpha (htra-2 alpha) & 7 & RNA splicing \\
\hline S & EXO1 & Hs.47504 & Exonuclease 1 & 6 & $\begin{array}{l}\text { DNA replication and } \\
\text { repair }\end{array}$ \\
\hline S & FEN1 & Hs.4756 & $\begin{array}{l}\text { Flap structure-specific } \\
\text { endonuclease } 1\end{array}$ & 7 & $\begin{array}{l}\text { DNA replication and } \\
\text { repair }\end{array}$ \\
\hline S & RAD51 & Hs.343807 & $\begin{array}{l}\text { RAD51 homolog (RecA homolog, } \\
\text { E. coli) (S. cerevisiae) }\end{array}$ & 8 & $\begin{array}{l}\text { DNA replication and } \\
\text { repair }\end{array}$ \\
\hline S & $R R M 1$ & Hs.2934 & $\begin{array}{l}\text { Ribonucleotide reductase M1 } \\
\text { polypeptide }\end{array}$ & 7 & $\begin{array}{l}\text { Purine and pyrimidine } \\
\text { metabolism }\end{array}$ \\
\hline S & ZNF217 & Hs. 155040 & Zinc finger protein 217 & 1 & Transcription \\
\hline $\mathrm{S}$ & ZWINT & Hs.42650 & ZW10 interactor & 7 & Unknown \\
\hline S & ESTs AL050331 & Hs.237225 & $\begin{array}{l}\text { Homo sapiens, clone MGC: } 24302 \\
\text { IMAGE:3996246, mRNA, complete } \\
\text { cds (hypothetical protein HTO23) }\end{array}$ & 3 & Unnamed \\
\hline$S$ & KIAA0101 & Hs.81892 & KIAA0101 gene product & 8 & Unnamed \\
\hline G2 & MUC1 & Hs.89603 & Mucin 1, transmembrane & 2 & $\begin{array}{l}\text { Cell communication, } \\
\text { adhesion or motility }\end{array}$ \\
\hline G2 & TUBA1 & Hs.75318 & Tubulin, alpha 1 (testis specific) & 7 & $\begin{array}{l}\text { Cell communication, } \\
\text { adhesion or motility }\end{array}$ \\
\hline G2 & TUBB & Hs.336780 & Tubulin, beta polypeptide & 7 & $\begin{array}{l}\text { Cell communication, } \\
\text { adhesion or motility }\end{array}$ \\
\hline G2 & STK12 & Hs.180655 & Serine/threonine kinase 12 & 7 & Cell cycle \\
\hline G2 & KNSL4 & Hs.119324 & Kinesin-like 4 & 8 & Cell cycle \\
\hline G2 & MAD2L1 & Hs.79078 & $\begin{array}{l}\text { MAD2 mitotic arrest deficient-like } 1 \\
\text { (yeast) }\end{array}$ & 8 & Cell cycle \\
\hline G2 & $C D C 25 C$ & Hs.656 & Cell division cycle 25C & 8 & Cell cycle \\
\hline G2 & PSMD11 & Hs.90744 & $\begin{array}{l}\text { Proteasome (prosome, macropain) } \\
26 \mathrm{~S} \text { subunit, non-ATPase, } 11\end{array}$ & 8 & Protein degradation \\
\hline G2 & HMG2 & Hs.80684 & $\begin{array}{l}\text { High-mobility group (nonhistone } \\
\text { chromosomal) protein } 2\end{array}$ & 6 & Transcription \\
\hline
\end{tabular}


Table 6 Continued

\begin{tabular}{|c|c|c|c|c|c|}
\hline 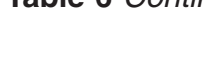 & Symbol & UniGene $^{c}$ & Gene description & Cluster $^{d}$ & Functione \\
\hline $\begin{array}{l}\text { Phase }{ }^{\mathrm{b}} \text { Cont } \\
\text { G2 }\end{array}$ & KPNA2 & Hs.159557 & $\begin{array}{l}\text { Karyopherin alpha } 2 \text { (RAG cohort } \\
1 \text {, importin alpha 1) }\end{array}$ & 8 & Transporters \\
\hline G2 & TMPO & Hs.11355 & Thymopoietin & 7 & Unknown \\
\hline G2 & KIAA0074 & Hs.1192 & KIÁA0074 protein & 8 & Unnamed \\
\hline $\mathrm{G} 2 / \mathrm{M}$ & $B \cup B 1 B$ & Hs.36708 & $\begin{array}{l}\text { BUB1 budding uninhibited by } \\
\text { benzimidazoles } 1 \text { homolog beta } \\
\text { (yeast) }\end{array}$ & 7 & Cell cycle \\
\hline G2/M & $C D C 20$ & Hs.82906 & $\begin{array}{l}\text { CDC20 cell division cycle } 20 \\
\text { homolog (S. cerevisiae) }\end{array}$ & 7 & Cell cycle \\
\hline G2/M & CENPA & Hs.1594 & Centromere protein $\mathrm{A}(17 \mathrm{kDa})$ & 7 & Cell cycle \\
\hline $\mathrm{G} 2 / \mathrm{M}$ & STK15 & Hs.250822 & Serine/threonine kinase 15 & 8 & Cell cycle \\
\hline $\mathrm{G} 2 / \mathrm{M}$ & KNSL 6 & Hs.69360 & $\begin{array}{l}\text { Kinesin-like } 6 \text { (mitotic } \\
\text { centromere-associated kinesin) }\end{array}$ & 8 & Cell cycle \\
\hline G2/M & CKS2 & Hs.83758 & CDC28 protein kinase 2 & 8 & Cell cycle \\
\hline G2/M & PLK & Hs.77597 & Polo-like kinase (Drosophila) & 8 & Cell cycle \\
\hline G2/M & BIRC5 & Hs.1578 & $\begin{array}{l}\text { Baculoviral IAP repeat-containing } 5 \\
\text { (survivin) }\end{array}$ & 8 & Cell death \\
\hline G2/M & $T S N$ & Hs.75066 & Translin & 7 & $\begin{array}{l}\text { DNA replication and } \\
\text { repair }\end{array}$ \\
\hline G2/M & $R A D 51 C$ & Hs.11393 & RAD51 homolog C (S. cerevisiae) & 8 & $\begin{array}{l}\text { DNA replication and } \\
\text { repair }\end{array}$ \\
\hline G2/M & $S F P Q$ & Hs.180610 & $\begin{array}{l}\text { Splicing factor proline/glutamine } \\
\text { rich (polypyrimidine tract binding } \\
\text { protein associated) }\end{array}$ & 8 & RNA splicing \\
\hline G2/M & TRIP13 & Hs.6566 & $\begin{array}{l}\text { Thyroid hormone receptor } \\
\text { interactor } 13\end{array}$ & 6 & Transcription \\
\hline G2/M & FOXM1 & Hs.239 & Forkhead box M1 & 8 & Transcription \\
\hline G2/M & ANP32B & Hs. 84264 & $\begin{array}{l}\text { Acidic (leucine-rich) nuclear } \\
\text { phosphoprotein } 32 \text { family, member } \\
\text { B }\end{array}$ & 8 & Unknown \\
\hline
\end{tabular}

aldentification of genes periodically expressed during the cell cycle was according to the gene expression data of Whitfield et al. (2002) (http://genome-www.stanford.edu/Human-CellCycle/HeLa/); ${ }^{b}$ cell cycle phase of maximum gene expression change in synchronously cycling HeLa cells (Whitfield et al. 2002); cUniGene cluster number; destrogen-responsive gene cluster (Fig. 2); efunction assigned according to Gene Ontology categories from LocusLink and/or GeneCards databases.

of higher eukaryotes, as it has also been observed in yeast cells, where genes with similar functions localize in adjacent positions along chromosomes (Cohen et al. 2000). These, and similar observations on locus control regions (LCRs) (Dekker 2003), point to the involvement of chromatin domains in the control of gene activity within genomic neighborhoods. At the same time, they suggest a higher order organization of genomes, according to which gene positioning in the chromosomes often reflects their expression in specific cell types, as well as their responsiveness to signals from the environment and the differentiation status of the cell. To our knowledge, this possibility has not been evaluated yet in the case of genes responsive to a hormone. We thus searched the cytogenetic location of each estrogen-regulated gene identified here, and then asked whether hormone-responsive gene distribution among chromosomes showed any imbalance. The results of this analysis are summarized in Tables 7 and 8, which showed (i) the predicted number of genes/chromosome and the number of genes within the chip which could be assigned to a specific cytogenetic location, identified manually on genome browsers (Table 7) or automatically by the web-based Onto-Express V2 software (Draghici et al. 2003, Table 8); (ii) the chromosomal distribution of the 344 hormoneregulated genes, obtained as above; (iii) a comparison between the predicted distribution of these genes among chromosomes, by random partitioning based exclusively on chromosomal gene density, and that determined experimentally, including statistical evaluation of these data by the 
Table 7 Chromosomal location of the estrogen responsive genes identified by genome-wide gene expression profiling in human breast cancer ZR-75.1 cells

\begin{tabular}{|c|c|c|c|c|c|c|}
\hline & Genome $^{a}$ & Analyzed $^{\mathrm{b}}$ & A. Predicted ${ }^{c}$ & B. Observed d & $B / A^{e}$ & $\chi^{2}$ test $^{f}$ \\
\hline \multicolumn{7}{|c|}{ Chromosome } \\
\hline 1 & 3533 & 702 & $34 \cdot 7$ & 35 & 1.01 & 0.9613 \\
\hline 2 & 2834 & 495 & 24.5 & 24 & 0.98 & 0.9188 \\
\hline 3 & 2191 & 386 & $19 \cdot 1$ & 12 & 0.63 & 0.0957 \\
\hline 4 & 1780 & 283 & $14 \cdot 0$ & 9 & 0.64 & 0.1703 \\
\hline 5 & 2021 & 352 & $17 \cdot 4$ & 21 & $1 \cdot 21$ & 0.3785 \\
\hline $6^{*}$ & 2106 & 351 & $17 \cdot 4$ & 26 & 1.49 & 0.0336 \\
\hline 7 & 2073 & 349 & $17 \cdot 3$ & 14 & 0.81 & 0.4198 \\
\hline 8 & 1604 & 251 & $12 \cdot 4$ & 12 & 0.97 & 0.9029 \\
\hline 9 & 1582 & 262 & $13 \cdot 0$ & 12 & 0.92 & 0.7837 \\
\hline 10 & 1654 & 261 & $12 \cdot 9$ & 17 & 1.32 & 0.2435 \\
\hline 11 & 2221 & 388 & $19 \cdot 2$ & 16 & 0.83 & 0.4541 \\
\hline 12 & 1855 & 399 & $19 \cdot 7$ & 23 & $1 \cdot 17$ & 0.4520 \\
\hline 13 & 865 & 133 & $6 \cdot 6$ & 2 & 0.30 & 0.0670 \\
\hline 14 & 1237 & 231 & 11.4 & 16 & 1.40 & 0.1656 \\
\hline 15 & 1365 & 213 & 10.5 & 13 & 1.24 & 0.4368 \\
\hline 16 & 1532 & 291 & 14.4 & 15 & 1.04 & 0.8708 \\
\hline $17^{*}$ & 1731 & 391 & $19 \cdot 3$ & 33 & 1.71 & 0.0015 \\
\hline 18 & 719 & 114 & $5 \cdot 6$ & 4 & 0.71 & 0.4786 \\
\hline $19^{*}$ & 1934 & 404 & $20 \cdot 0$ & 10 & 0.50 & 0.0219 \\
\hline 20 & 973 & 176 & 8.7 & 7 & 0.80 & 0.5527 \\
\hline 21 & 457 & 84 & 4.2 & 4 & 0.95 & 0.9373 \\
\hline 22 & 833 & 139 & 6.9 & 4 & 0.58 & 0.2604 \\
\hline $\mathrm{x}$ & 1524 & 247 & $12 \cdot 2$ & 12 & 0.98 & 0.9482 \\
\hline Y & 248 & 10 & 0.5 & 1 & 2.00 & 0.4613 \\
\hline Ug & 242 & 981 & - & 2 & - & - \\
\hline Total & 39114 & 7893 & (342) & 344 & - & 1.0000 \\
\hline
\end{tabular}

aGene assignment to specific chromosomes according to The Weizmann Institute's Unified Database for human genome mapping (http://bioinformatics.weizmann.ac.il/udb_25/); b genes analyzed (spotted DNAs which pass QCs tests during microarray fabrication and fluorescence image analysis); ${ }^{\circ}$ calculated considering the number of genes analyzed, their partitioning among chromosomes and the number of estrogen-regulated genes detected experimentally; dnumber of estrogen regulated genes per chromosome (according to GeneCards); eratio of the number of genes observed vs number of genes predicted; fprobability associated with $\chi^{2}$ test (chromosomes where results suggest the highest probability of non-random density of estrogen-regulated genes are marked by

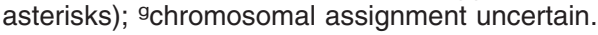

$\chi^{2}$ test (Table 7); (iv) significance analysis of the chromosomal distribution of estrogen-regulated genes among chromosomes, calculated by hypergeometric probability distribution (Table 8). When combined, the results of these studies show that hormone-responsive gene density is significantly higher in chromosomes 6 and $17(P=0.0342$ and 0.002 respectively) and lower in chromosome 19 $(P=0.0219)$. This, which was confirmed by direct computation of data within each chromosome (Table 7) as well as by comparative evaluations across all chromosomes (Table 8), provides strong evidence supporting the possibility of a higher order organization of the hormone-regulated transcriptome within the genome. Accordingly, genes regulated either directly or indirectly by estrogen in BC cells might cluster together in discrete chromosomal domains. It is worth mentioning that this is not unprecedented, as several examples of coordinated gene regulation within defined chromosomal domains are known. This is the case, for example, of the rRNA, histone, homeobox or globin gene clusters, where colinearity reflects important features of the physiological role of the genes involved and the genetic mechanisms allowing their regulation. Interestingly, expression of certain Hox gene clusters is controlled also by retinoids (Boncinelli et al. 1991), which like estrogen - act via nuclear receptors (RARs and RXRs). A considerable fraction of the hormoneregulated genes identified here (close to $10 \%$ of the total) are localized on the relatively small 


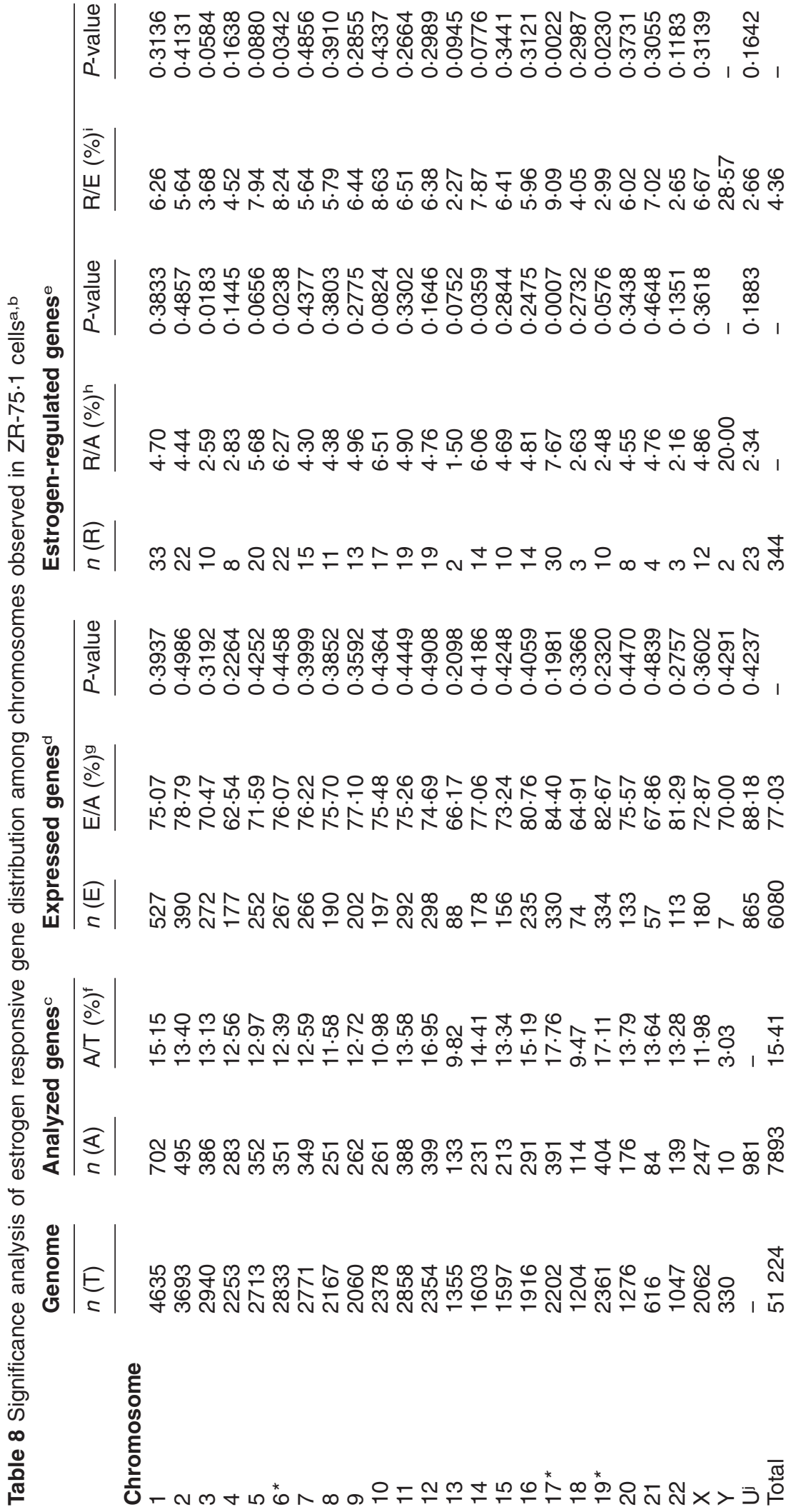

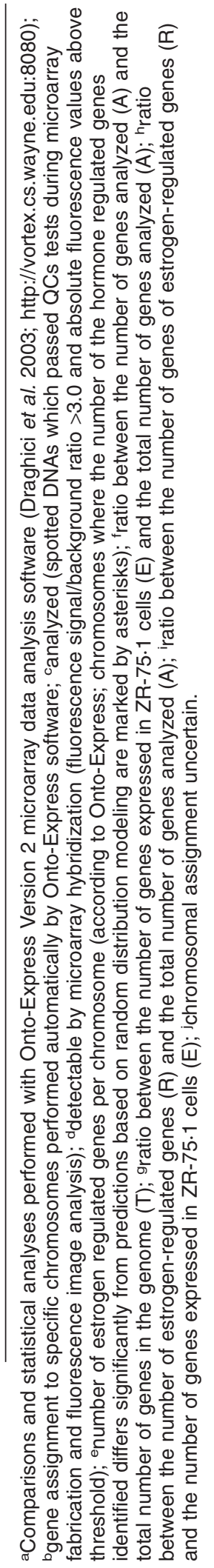


Table 9 Distribution of estrogen $(E)$ responsive genes from breast cancer cells within chromosome 17

\begin{tabular}{|c|c|c|c|c|}
\hline & $\begin{array}{l}\text { No. of genes } \\
n(T)\end{array}$ & $\begin{array}{l}\text { No. of E responsive genes } \\
n(\mathrm{R})\end{array}$ & $\mathrm{R} / \mathrm{T}(\%)$ & Cytogenetica \\
\hline \multicolumn{5}{|c|}{ Interval from pter (Mb) } \\
\hline $0 \sim 4$ & 104 & 4 & 3.85 & \\
\hline $4 \sim 8$ & 141 & 13 & $9 \cdot 22$ & 17p13.1-p13.3 \\
\hline $8 \sim 12$ & 74 & 5 & $6 \cdot 76$ & \\
\hline $12 \sim 16$ & 45 & 1 & $2 \cdot 22$ & \\
\hline $16 \sim 20$ & 114 & 3 & $2 \cdot 63$ & \\
\hline 20 25 & 45 & 1 & $2 \cdot 22$ & \\
\hline \multicolumn{5}{|c|}{ centromere } \\
\hline $25 \sim 29$ & 107 & 2 & $1 \cdot 87$ & \\
\hline $29 \sim 33$ & 49 & 3 & $6 \cdot 12$ & \\
\hline $33 \sim 37$ & 105 & 2 & 1.90 & \\
\hline $37 \sim 41$ & 179 & 4 & $2 \cdot 23$ & \\
\hline $41 \sim 45$ & 133 & 13 & $9 \cdot 77$ & $17 q 21.2-p 21.3$ \\
\hline $45 \sim 49$ & 112 & 5 & 4.46 & \\
\hline $49 \sim 53$ & 20 & 7 & 35.00 & $17 q 21.33$ \\
\hline $53 \sim 57$ & 56 & 0 & 0.00 & \\
\hline $57 \sim 61$ & 60 & 7 & 11.67 & $17 q 22-q 23$ \\
\hline $61 \sim 65$ & 62 & 3 & 4.84 & \\
\hline $65 \sim 69$ & 55 & 3 & 5.45 & \\
\hline $69 \sim 73$ & 59 & 2 & 3.39 & \\
\hline 73 77 & 117 & 5 & $4 \cdot 27$ & \\
\hline$>77$ & 94 & 12 & $12 \cdot 77$ & $17 q 25.2-q 25.3$ \\
\hline Total & 1731 & 95 & $100 \cdot 00$ & \\
\hline
\end{tabular}

aFrom Ensembl and/or UCSC Golden Path databases; bresponse to estrogen in breast cancer cells known and/or assessed by microarray analysis.

chromosome 17, despite the fact that this carries only $\sim 5 \%$ of the genes expressed at detectable levels in ZR-75.1 cells (Tables 7 and 8). Interestingly, this chromosome frequently shows gross abnormalities in $\mathrm{BC}$ (deletions and, more often, amplifications in 15 to $25 \%$ of the cases; Forozan et al. 2000), which are thought to be involved in malignant transformation of these cells and/or to be determinant for the tumor phenotype. We thus focused our attention on this chromosome and determined the physical localization of its estrogen-regulated genes, including those identified in this study and nine additional ones known to be regulated by $\mathrm{E} 2$ in $\mathrm{BC}$ cells (BRCA1, ERBB2, KRT19, MPO, TP53, TRIM16, SHBG, WNT3 and ZNF147; Yamada et al. 1993, Inoue et al. 1995, Xu et al. 1997, Liu et al. 1998, Murayama et al. 1999, Choi et al. 2000, Perissi et al. 2000, Qin et al. 2002, Katoh 2003). In addition, we searched for more such genes in the published datasets from microarray and/or SAGE analyses of the hormone-regulated transcriptome of $\mathrm{BC}$ cells (Charpentier et al. 2000, Finlin et al. 2001, Bouras et al. 2002, Inoue et al. 2002, Lobenhofer et al. 2002,
Hodges et al. 2003) and from microarray expression data from estrogen-treated ZR-75·1 and MCF-7 cells available from our laboratories (A Weisz, unpublished data). This led to the identification of 95 hormone-regulated genes whose distribution within chromosome 17 is reported, at $4 \mathrm{Mbs}$ resolution, in Table 9. When compared with the local gene density ( $\mathrm{R} / \mathrm{T}$ ratios in Table 9), distribution of these hormone-responsive transcription units along the chromosome does not appear uniform. The average density of such genes within the centromeric region between cytogenetic loci $17 \mathrm{p} 11 \cdot 2$ and $17 \mathrm{q} 21 \cdot 2-\mathrm{q} 21 \cdot 3(12 \sim 40 \mathrm{Mbs}$ interval from $\mathrm{pter})$, for example, is much lower $(2 \cdot 74 \%)$ than that of the more telomeric regions of either $17 \mathrm{p}$ or $17 \mathrm{q}(6 \cdot 61 \%$ and $9 \cdot 16 \%$ respectively). Furthermore, we observed some distribution outliers, in particular within $17 \mathrm{p} 13 \cdot 1-\mathrm{p} 13 \cdot 3,17 \mathrm{q} 21 \cdot 33,17 \mathrm{q} 22-\mathrm{q} 23$ and $17 \mathrm{q} 25 \cdot 2-\mathrm{q} 25 \cdot 3$, where the hormone-responsive gene density is much higher than the average. All these data suggest the possible existence of estrogen-responsive domains in chromosome 17, an interesting novelty concerning the relationships between gene regulation by steroid hormones and 
genome architecture and functional organization. Indeed, in several instances hormone-regulated genes appear to cluster together on this chromosome (Table 10). For example, ten hormoneresponsive genes are concentrated within $\sim 1.71$ Mbs of $17 \mathrm{p} 13$, including ITGAE, ATP2A3 and KIAA0399 which cluster in a $<400 \mathrm{Kbs}$ subregion, and CXCL16, PSMB6, PLD2, GP1BA and ENO3, which are closely spaced within $\sim 200$ Kbs. Interestingly, in the same cytogenetic band $\mathcal{N}$ UP8o and C1 QBP genes from cluster 7 (Fig. 2b) are only $13 \mathrm{Kbs}$ apart from each other on the same DNA strand, while five other genes which also cluster together in the same cytogenetic location (EIF4A1, SHBG, TP53, VAMP2 and STK12) are all activated by estrogen, suggesting two possible examples of colinear gene regulation. Several other examples of physical clustering of co-regulated genes can be found also in $17 \mathrm{q}$, including the three keratin genes KRT13, KRT15 and KRT17, TUBG1, PSME3, BRCA1 and NBR2 or NPEPPS and KPNB1 at 17q21, TOB1, NME1 and NME2, SFRS1, MPO and $R A D 51 C$ or RPS6 KB1, NY-REN-60 and PPM1D at 17q23, KPNA2, LOC51321 and SLC16A6, LLGL2, ITGB4 and GALK1 or TK1, BIRC5 and SSI3/ SOCS3 at 17q25 (Table 10). These observations, which arise from a zoom-in on the estrogenresponsive transcriptome from chromosome 17 and are likely to apply also to other cytogenetic locations (see Tables 7 and 8), while awaiting further confirmation by whole genome studies of the estrogen-responsive transcriptomes from different cell types, already suggest some intriguing possibilities. Clustering of estrogen-responsive genes, for example, could provide a structural basis to allow them to respond in a coordinated fashion during development, cell differentiation or cell growth, as recently suggested for other gene expression 'neighborhoods' (Oliver et al. 2002). In this case, an epigenetic control of estrogenresponsive gene clusters could involve locus-specific gene regulation domains, where the chromatin can shift between 'closed' and 'open' conformations allowing coordinated silencing/activation of many genes located in proximity to each other. In this respect, long-ranging chromatin unfolding by ER- $\alpha$ has recently been demonstrated experimentally (Nye et al. 2002), while the involvement of chromatin remodeling factors and histone modifying enzymes in nuclear receptor - incuding ER actions on target genes is well known. It would be interesting to verify now whether these co-regulated chromatin domains contain hormone-sensitive LCR elements. Furthermore, epigenetic control of the activity of genes and gene clusters is also the basis of genetic imprinting (Reik \& Walter 2001), which is suggested as one of the mechanisms responsible for genetic predisposition to cancer, including BC (Schofield et al. 2001). Chromosomal clustering of hormone-responsive genes can also be envisioned to provide a functional link with certain genomic imbalances frequently observed in tumor cells. BC cells, including ZR-75·1 and MCF-7 cells, show numerous karyotypic aberrations, in particular amplification and deletion of large chromosomal regions (Forozan et al. 2000). Interestingly, in the case of $17 \mathrm{q} 23$, amplification results in overexpression of many genes included in the amplified segment (Barlund et al. 2000, Monni et al. 2001). Thus, it is possible to envisage that the nonrandom distribution of estrogen-regulated genes observed in this study might reflect chromosomal imbalances in ZR-75·1 cells (Forozan et al. 2000). Amplification, for example, could lead to 'gene dosage' effects causing differences in the number of genes detectable by expression analysis between diploid and aneuploid chromosomal segments. In turn, this suggests also how clustering of responsive genes in defined genomic loci might play a role in hormone-responsive breast carcinogenesis and tumor progression. In fact, significant modifications of cell sensitivity to estrogens could result from amplification and deletion of chromosomal segments rich in hormone-responsive genes.

All these exciting hypotheses can now be effectively addressed, as the list of genes identified here and in other similar investigations aimed at mapping the hormone-regulated transcriptomes of responsive cell types can be exploited in silico to pinpoint potential chromosomal domains with strong evidence of colinear gene regulation, which may then be studied experimentally in model systems to identify, for example, primary and secondary gene responses to ERs within each cluster, as well as potential functional hierarchies among co-regulated genes and their contribution towards defined phenotypic modifications occurring in hormone-stimulated cells.

In conclusion, the gene expression changes described here represent a comprehensive view of the gene expression program activated by estrogen 


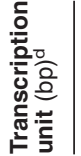

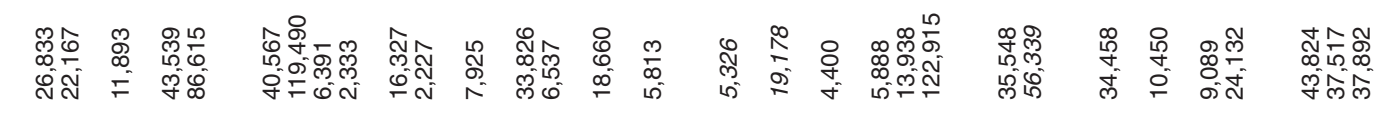

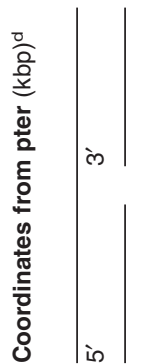

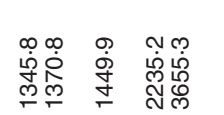

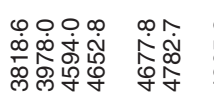

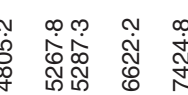

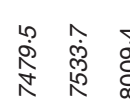

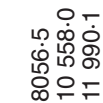

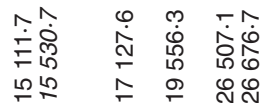

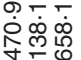
Nํำ

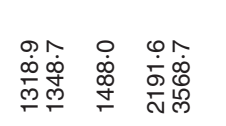

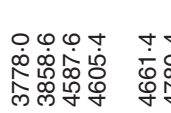

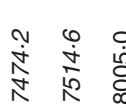

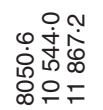

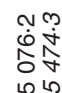

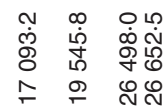

זㅇำ 襄
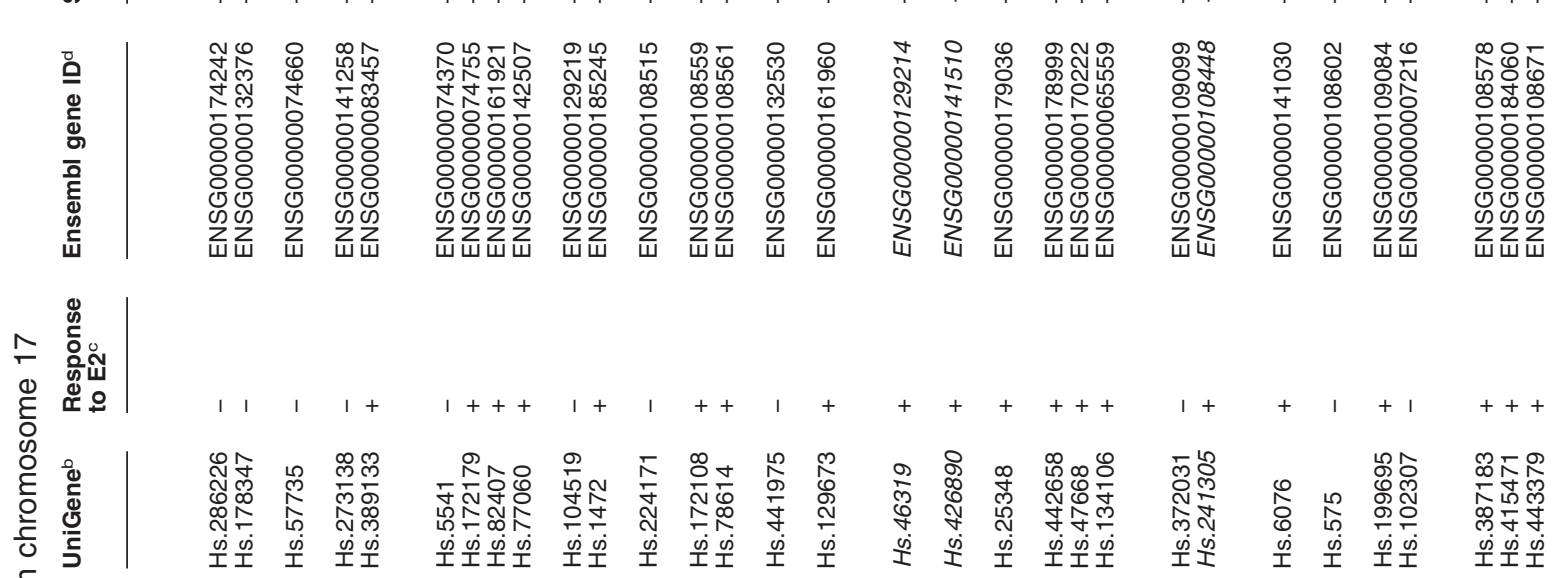

은

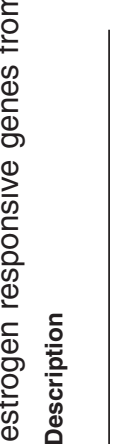

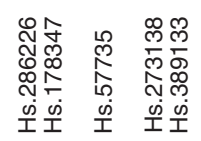

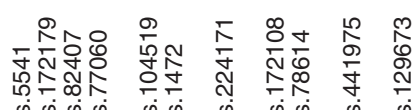

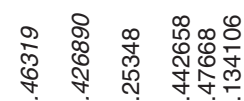

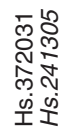

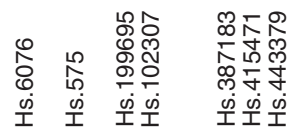
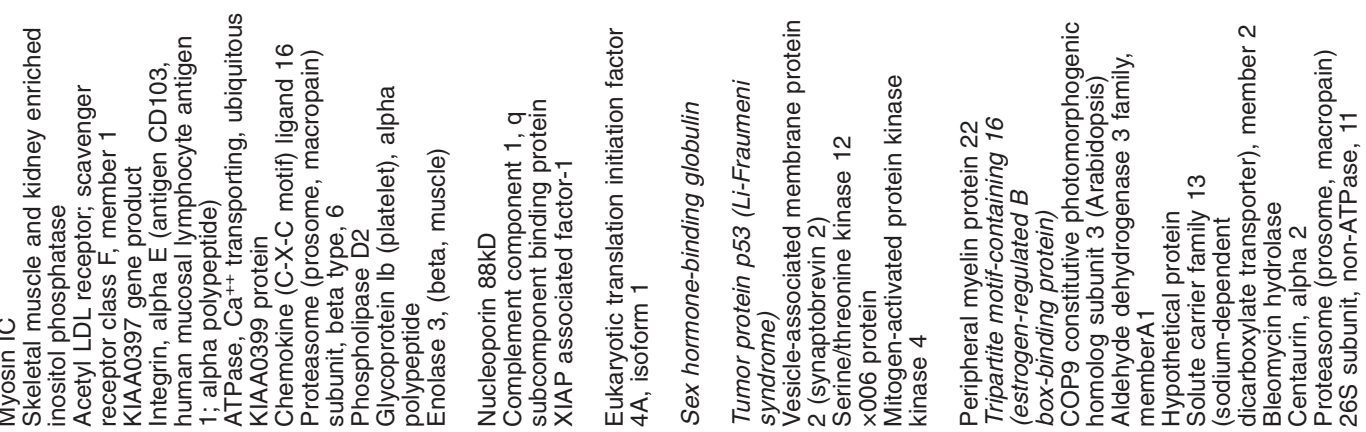

¿

등

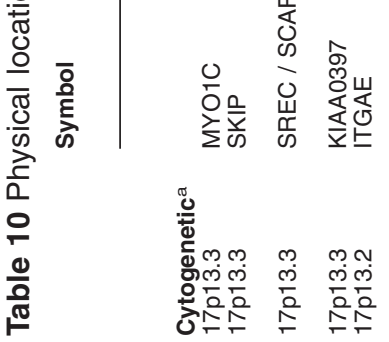

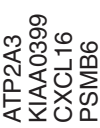

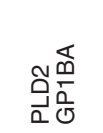

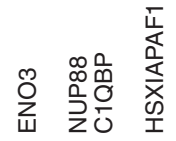

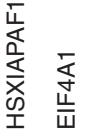

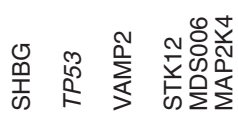

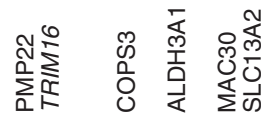

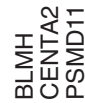

ำ 


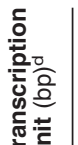

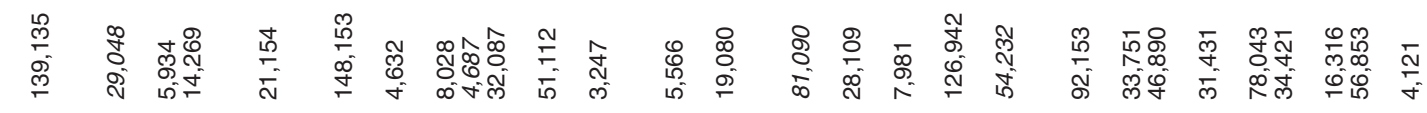

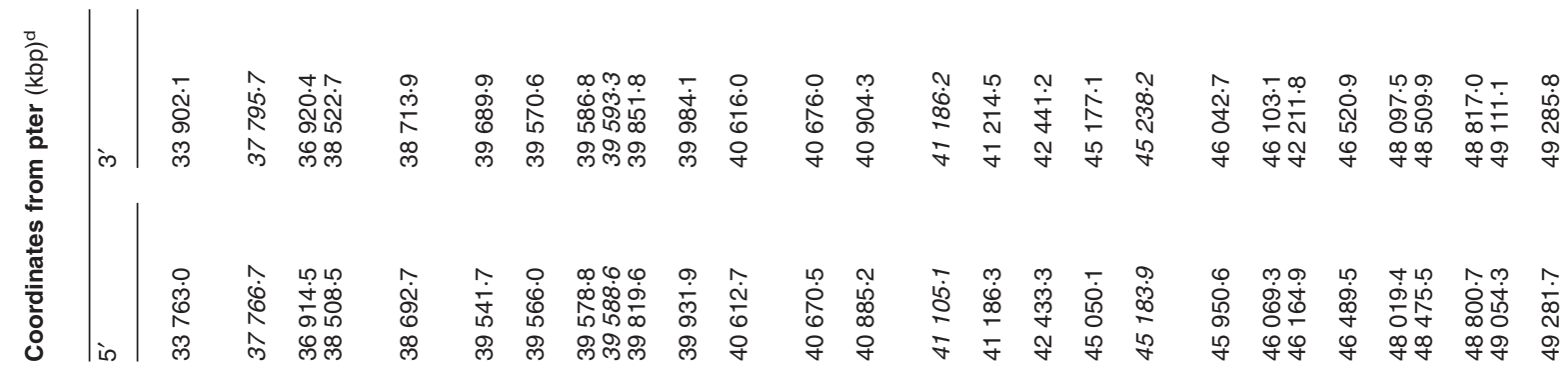

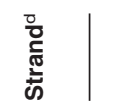
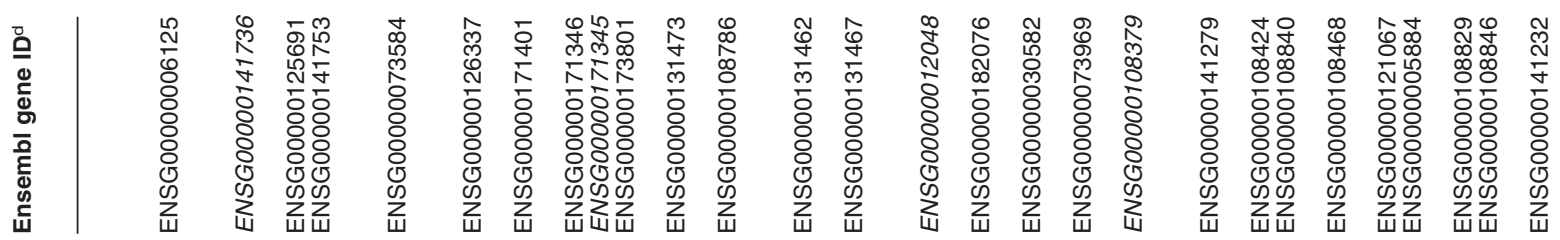

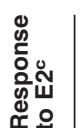

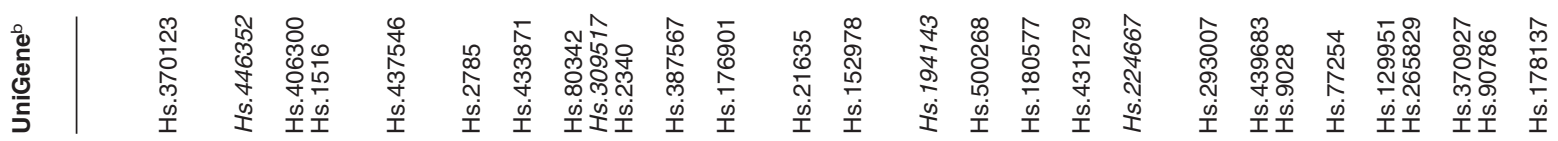
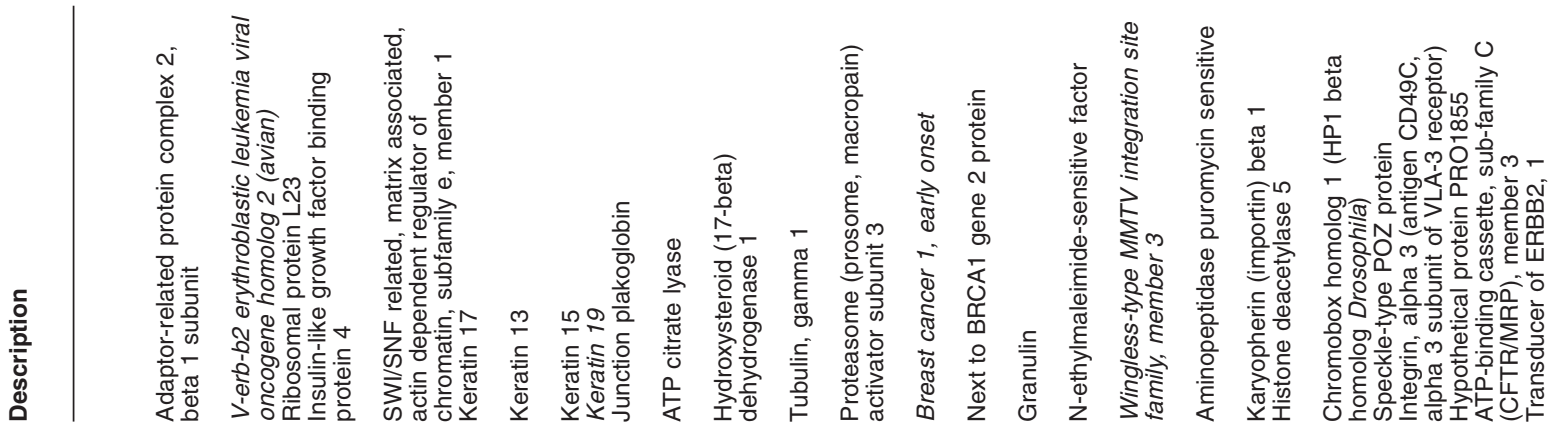

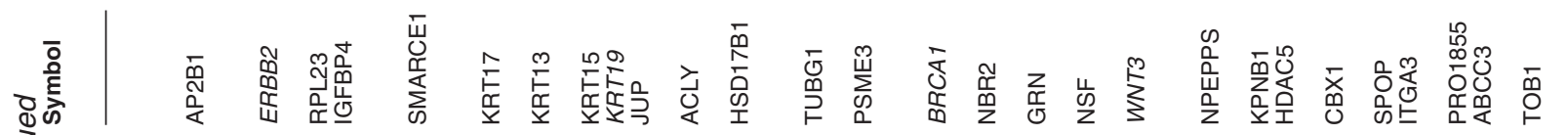

竞

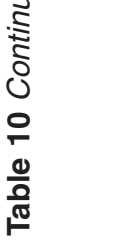

ธัँ

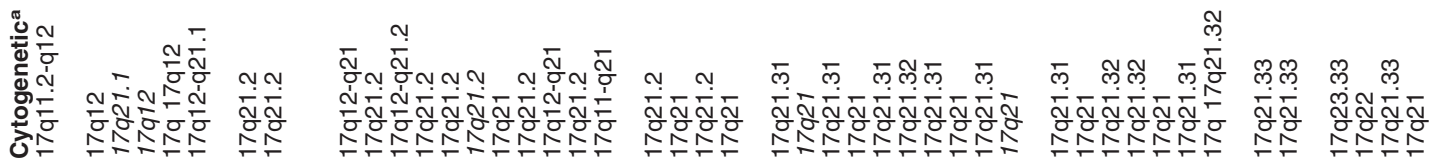




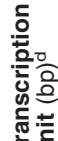

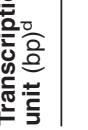

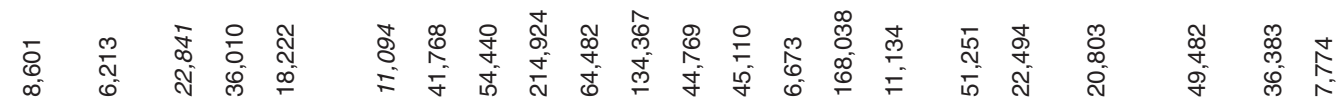

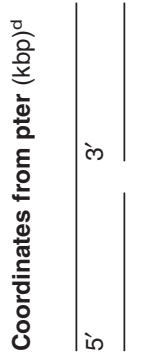

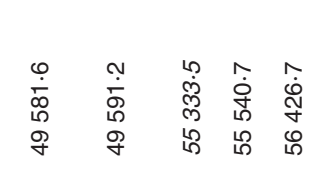

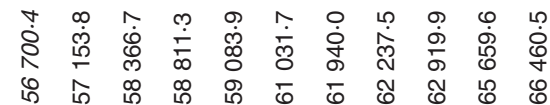

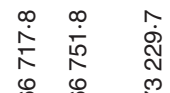

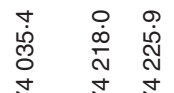

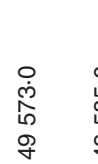

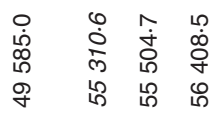

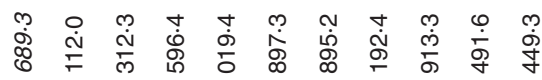

$\begin{array}{lll}0 & \infty & \infty \\ \dot{0} & \stackrel{\infty}{N} & \stackrel{\infty}{N} \\ 0 & 0 & 0\end{array}$

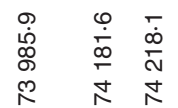

竞

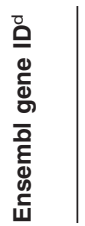

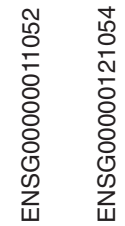

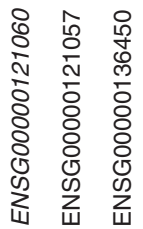

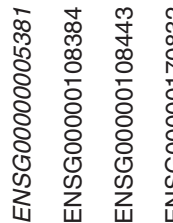

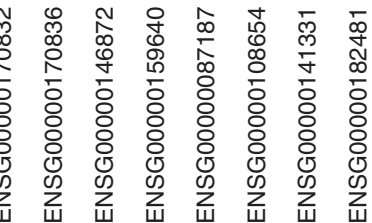

$8: 8$

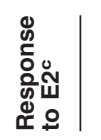

竞|

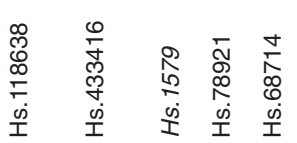

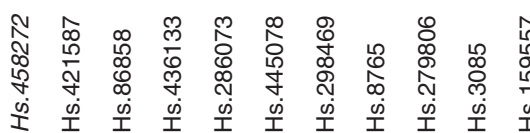

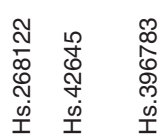

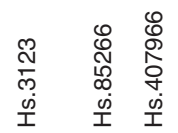
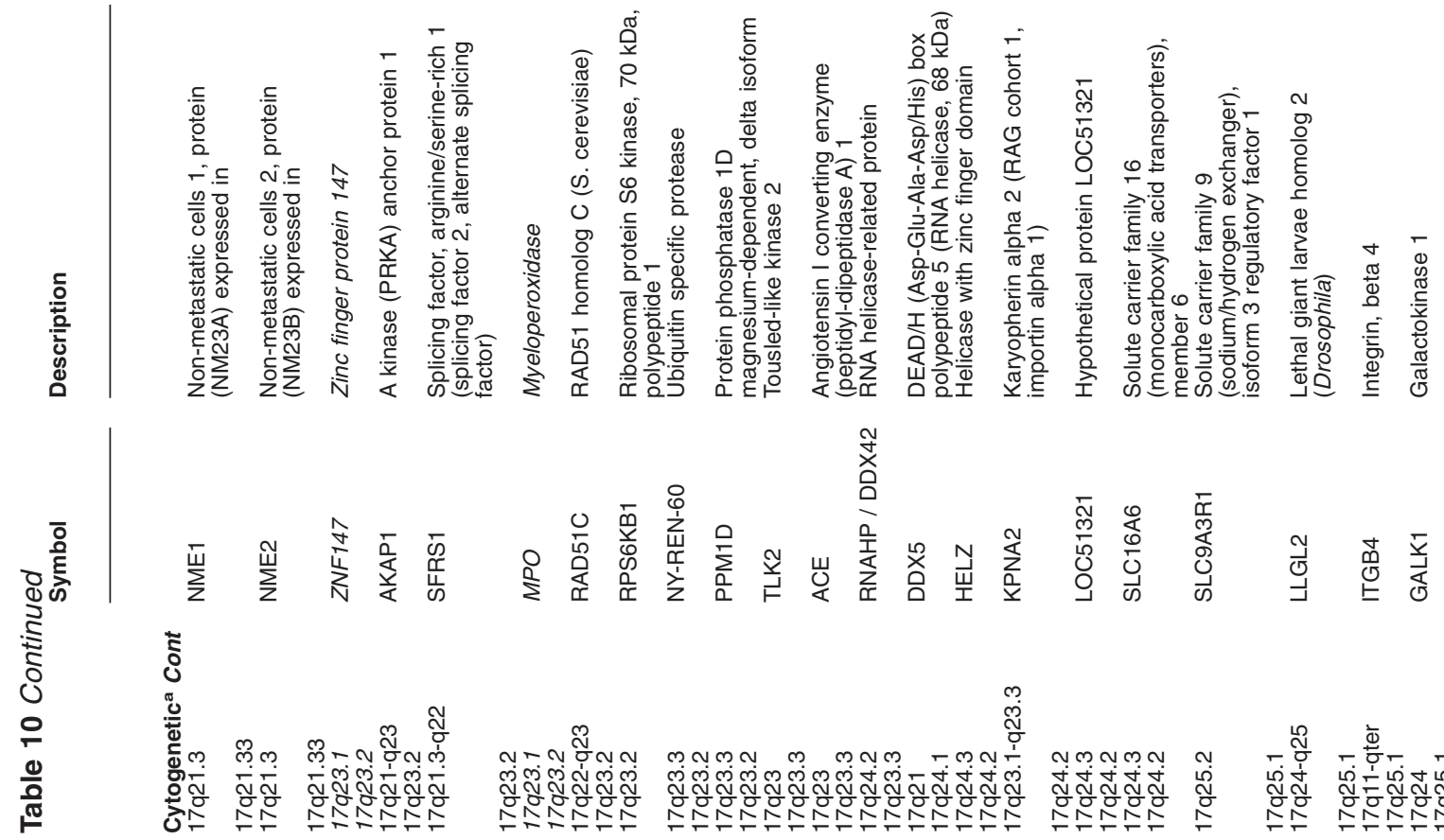

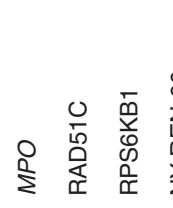

華

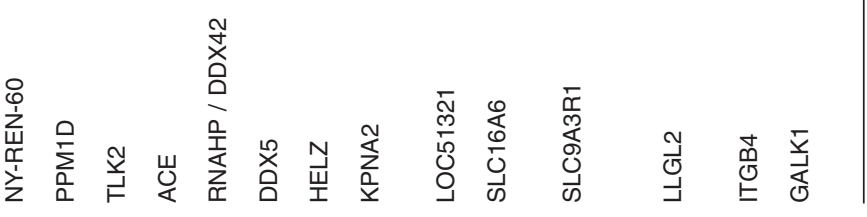

www.endocrinology.org

Journal of Molecular Endocrinology (2004) 32, 719-775 


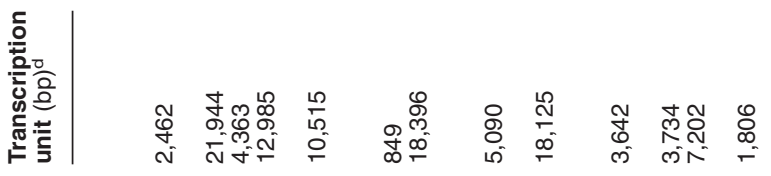

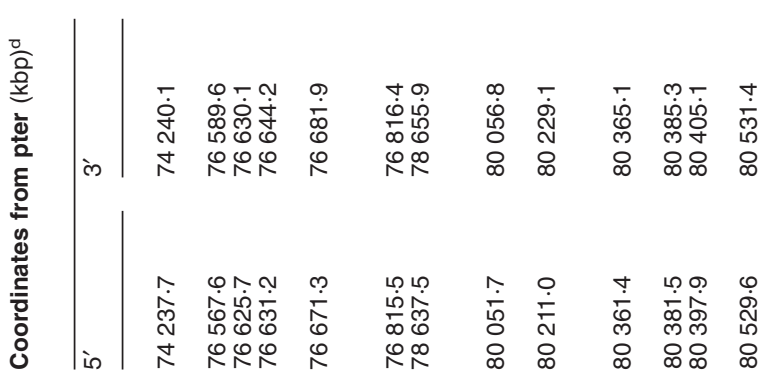

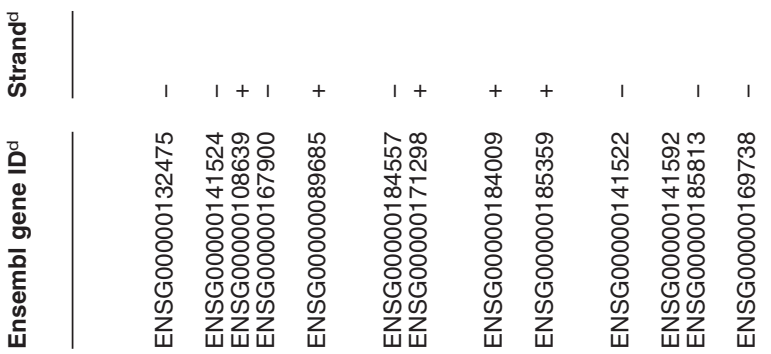

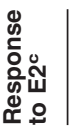

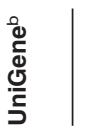

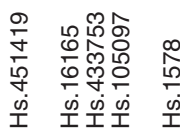

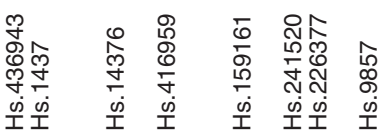

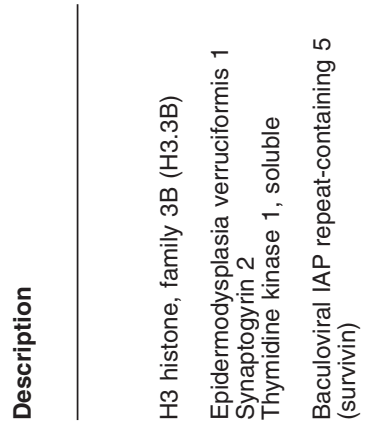

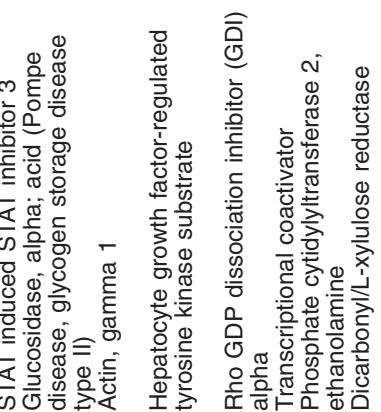
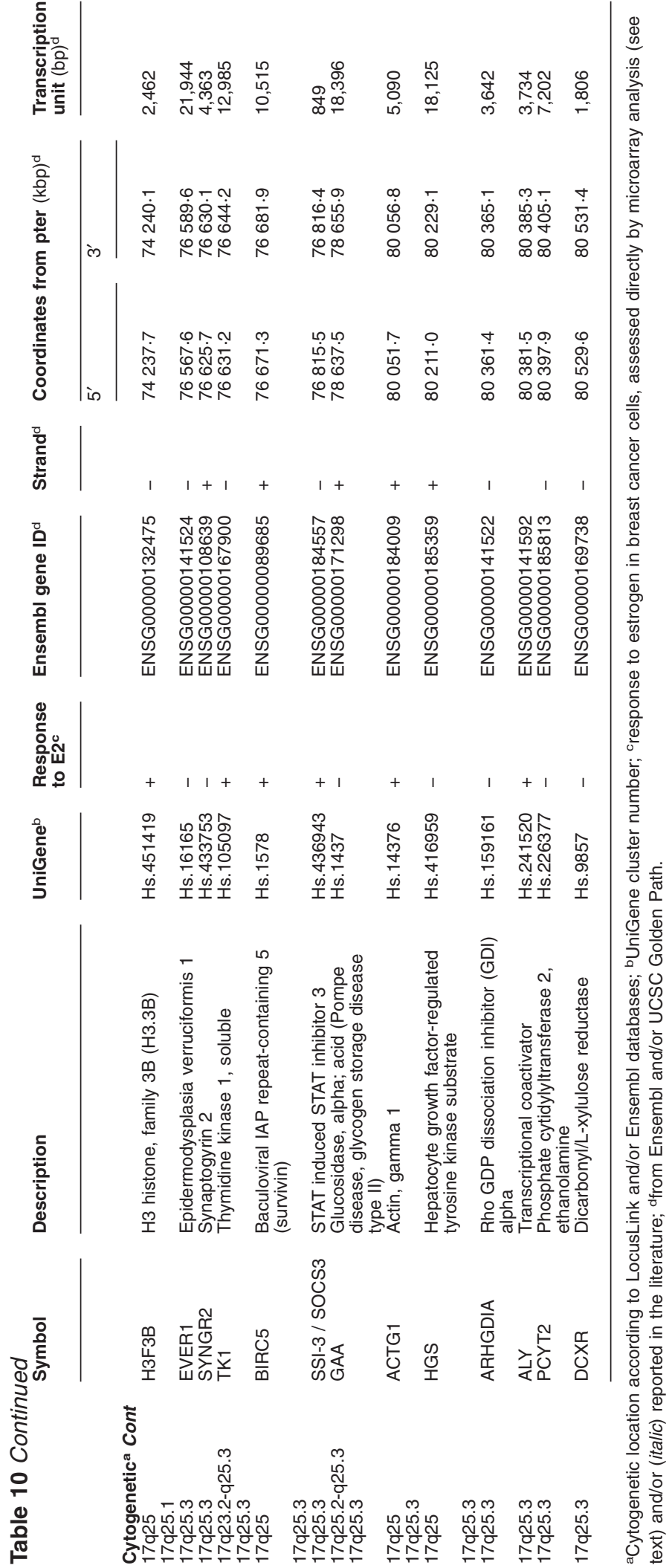
in a hormone-responsive $\mathrm{BC}$ cell line in vitro, which can be used to investigate various aspects of estrogen involvement in breast carcinogenesis and tumor progression and to further our understanding of the molecular and genetic basis of the responsiveness of $\mathrm{BC}$ cells to hormones and antihormones. A detailed in silico categorization and functional analysis of this estrogen-regulated gene set will provide a logical framework to pinpoint potential novel aspects of estrogen's actions in BC cells, which may then be tested experimentally and lead in this way to the identification of new biological properties of hormone-responsive cells. At the same time, the pattern of expression of dominant estrogen-regulated genes may characterize defined, and clinically useful, hormoneresponsive $\mathrm{BC}$ subtypes. It is reasonable to assume that a sizeable fraction of the gene expression profiles measured in hormone-stimulated BC cells in vitro reflects distinctive properties of these cells which, ultimately, may be exploited to type them ex vivo. Indeed, we have already shown that the expression pattern of some of the genes identified in this study clearly discriminates estrogen-responsive from -non-responsive $\mathrm{BC}$ cells in vitro (Weisz et al. 2003) and ER- $\alpha$-positive and -negative breast tumor samples (Sorbello et al. 2003).

\section{Acknowledgements}

We thank Salvatore Salzano for assistance in flow cytometry cell cycle analysis, Filomena Matarese and Roberta Penta for technical assistance with cell cultures, Laura Sacerdote (Università degli Studi di Torino) and Giulio Antoniol (Università del Sannio, Benevento) for suggestions and help in statistical analyses, Kim Hirshman (Open Channel Software, Chicago, IL, USA) and Sorin Draghici (Wayne State University, Detroit, MI, USA) for assistance with Onto-Express analysis, Shin-ichi Hayashi (Saitama Cancer Center Research Institute, Japan) for sharing microarray data, and Concetta Ambrosino for critically reading the manuscript.

The research was supported by the Italian Association for Cancer Research (AIRG, Investigator Grants 2002) to A W and P S, Italian Ministry for Education, University and Research: PRIN 2000-01 and 2001-02 (Grants 20011067229_002 and 2002067514_002) and FIRB Post-genomica (Grant RBNE0157EH_001), European Commission (Contracts BMH4-CT98-3433, QLG1-CT2000-01935 and QLK3-CT-2002-02029), Ministry of Health (Progetti Speciali 2000 and RF02/184), Regione Campania and Second University of Naples (Ricerca di Ateneo 2002), to A W, F B and L A; Regione Piemonte (Progetto Sanità Pubblica 1999 e 2000) and University of Torino (Ricerca di Ateneo), to M D B and P S; Consiglio Nazionale delle Ricerche: Progetto Finalizzato 'Biotecnologie', to R C.

C Scafoglio is a PhD student of Dottorato di Ricerca in Oncologia medica e chirurgica ed Immunologia clinica $\left(\mathrm{XVIII}^{\circ}\right.$ ciclo), Seconda Università degli Studi di Napoli.

\section{References}

Alizadeh AA, Eisen MB, Davis RE, Ma C, Lossos IS, Rosenwald A, Boldrick JC, Sabet H, Tran T, Yu X et al. 2000 Distinct types of diffuse large B-cell lymphoma identified by gene expression profiling. Nature 403 503-511.

Altucci L, Addeo R, Cicatiello L, Dauvois S, Parker MG, Truss M, Beato M, Sica V, Bresciani F \& Weisz A 1996 17 $\beta$-Estradiol induces cyclin D1 gene transcription, p36D1-p34 cdk4 complex activation and $\mathrm{p} 105 \mathrm{Rb}$ phosphorylation during mitogenic stimulation of $\mathrm{G}(1)$-arrested human breast cancer cells. Oncogene 12 2315-2324.

Ashburner M, Ball CA, Blake JA, Botstein D, Butler H, Cherry JM, Davis AP, Dolinski K, Dwight SS, Eppig JT et al. 2000 Gene ontology: tool for the unification of biology. The Gene Ontology Consortium. Nature Genetics 25 25-29.

Barlund M, Monni O, Kononen J, Cornelison R, Torhorst J, Sauter G, Kallioniemi OP \& Kallioniemi A 2000 Multiple genes at 17 q23 undergo amplification and overexpression in breast cancer. Cancer Research 60 5340-5344.

Blondel M \& Mann C 1996 G2 cyclins are required for the degradation of G1 cyclins in yeast. Nature 384 279-282.

Bonapace IM, Addeo R, Altucci L, Cicatiello L, Bifulco M, Laezza C, Salzano S, Sica V, Bresciani F \& Weisz A 1996 17 $\beta$-Estradiol overcomes a G1 block induced by HMG-CoA reductase inhibitors and fosters cell cycle progression without inducing ERK-1 and -2 MAP kinases activation. Oncogene 12 753-763.

Boncinelli E, Simeone A, Acampora D \& Mavilio F 1991 HOX gene activation by retinoic acid. Trends in Genetics 7 329-334.

Bortoluzzi S, Rampoldi L, Simionati B, Zimbello R, Barbon A, d'Alessi F, Tiso N, Pallavicini A, Toppo S, Cannata N et al. 1998 A comprehensive, high-resolution genomic transcript map of human skeletal muscle. Genome Research 8 817-825.

Bouras T, Southey MC, Chang AC, Reddel RR, Willhite D, Glynne R, Henderson MA, Armes JE \& Venter DJ 2002 Stanniocalcin 2 is an estrogen-responsive gene coexpressed with the estrogen receptor in human breast cancer. Cancer Research 62 1289-1295.

Caron H, van Schaik B, van der Mee M, Baas F, Riggins G, van Sluis P, Hermus MC, van Asperen R, Boon K, Voute PA et al. 2001 The human transcriptome map: clustering of highly expressed genes in chromosomal domains. Science 291 1289-1292. 
Charpentier AH, Bednarek AK, Daniel RL, Hawkins KA, Laflin KJ, Gaddis S, MacLeod MC \& Aldaz CM 2000 Effects of estrogen on global gene expression: identification of novel targets of estrogen action. Cancer Research 60 5977-5983.

Choi I, Gudas LJ \& Katzenellenbogen BS 2000 Regulation of keratin 19 gene expression by estrogen in human breast cancer cells and identification of the estrogen responsive gene region. Molecular and Cellular Endocrinology 164 225-237.

Cicatiello L, Addeo R, Altucci L, Belsito Petrizzi V, Boccia V, Cancemi M, Germano D, Pacilio C, Salzano S, Bresciani F et al. 2000 The antiestrogen ICI 182,780 inhibits proliferation of human breast cancer cells by interfering with multiple, sequential estrogen-regulated processes required for cell cycle completion. Molecular and Cellular Endocrinology 165 199-209.

Cohen BA, Mitra RD, Hughes JD \& Church GM 2000 A computational analysis of whole-genome expression data reveals chromosomal domains of gene expression. Nature Genetics 26 183-186.

Couillard S, Labrie C, Gauthier S, Merand Y, Singh SM, Candas B \& Labrie F 2000 Long-term inhibitory effect of the orally active and pure antiestrogen EM-800 on the growth of human breast cancer xenografts in nude mice. International fournal of Cancer $\mathbf{8 5}$ $424-429$.

Dekker J 2003 A closer look at long-range chromosomal interactions. Trends in Biochemical Sciences 28 277-280.

Draghici S, Khatri P, Martins RP, Ostermeier GC \& Krawetz SA 2003 Global functional profiling of gene expression. Genomics $\mathbf{8 1}$ 98-104.

Dudoit S, Yang YH, Callow MJ \& Speed TP 2001 Statistical methods for identifying differentially expressed genes in replicated cDNA microarray experiments. Technical report \#578. http://www.stat.berkeley.edu/users/terry/zarray/Html/matt.html.

Eisen MB, Spellman PT, Brown PO \& Botstein D 1998 Cluster analysis and display of genome-wide expression patterns. PNAS 95 14863-14868.

Fambrough D, McClure K, Kazlauskas A \& Lander ES 1999 Diverse signaling pathways activated by growth factor receptors induce broadly overlapping, rather than independent, sets of genes. Cell 97 727-741.

Finlin BS, Gau CL, Murphy GA, Shao H, Kimel T, Seitz RS, Chiu YF, Botstein D, Brown PO, Der CJ et al. 2001 Rerg is a novel ras-related, estrogen-regulated and growth-inhibitory gene in breast cancer. Fournal of Biological Chemistry 276 42259-42267.

Forozan F, Mahlamaki EH, Monni O, Chen Y, Veldman R, Jiang Y, Gooden GC, Ethier SP, Kallioniemi A \& Kallioniemi OP 2000 Comparative genomic hybridization analysis of 38 breast cancer cell lines: a basis for interpreting complementary DNA microarray data. Cancer Research 60 4519-4525.

Frasor J, Danes JM, Komm B, Chang K, Lyttle CR \& Katzenellenbogen BS 2003 Profiling of estrogen up- and down-regulated gene expression in human breast cancer cells: insights into gene networks and pathways underlying estrogenic control of proliferation and cell phenotype. Endocrinology 144 4562-4574.

Gene Expression Group web site: http://crisceb.unina2.it/geneexpression/

Hedenfalk I, Duggan D, Chen Y, Radmacher M, Bittner M, Simon R, Meltzer P, Gusterson B, Esteller M, Kallioniemi OP et al. 2001 Gene-expression profiles in hereditary breast cancer. New England Fournal of Medicine 344 539-548.

Heid CA, Stevens J, Livak KJ \& Williams PM 1994 Real time quantitative PCR. Genome Research 6 986-994.

Hodges LC, Cook JD, Lobenhofer EK, Li L, Bennett L, Bushel PR, Aldaz CM, Afshari CA \& Walker CL 2003 Tamoxifen functions as a molecular agonist inducing cell cycle-associated genes in breast cancer cells. Molecular Cancer Research $1300-311$.
Horne MC, Donaldson KL, Goolsby GL, Tran D, Mulheisen M, Hell JW \& Wahl AF 1997 Cyclin G2 is up-regulated during growth inhibition and $\mathrm{B}$ cell antigen receptor-mediated cell cycle arrest. Fournal of Biological Chemistry 272 12650-12661.

Inoue A, Yoshida N, Omoto Y, Oguchi S, Yamori T, Kiyama R \& Hayashi S 2002 Development of cDNA microarray for expression profiling of estrogen-responsive genes. Fournal of Molecular Endocrinology 29 175-192.

Inoue S, Orimo A, Matsuda Y, Inazawa J, Emi M, Nakamura Y, Hori T \& Muramatsu M 1995 Chromosome mapping of human (ZNF147) and mouse genes for estrogen-responsive finger protein (efp), a member of the RING finger family. Genomics 25 581-583.

Iyer VR, Eisen MB, Ross DT, Schuler G, Moore T, Lee JC, Trent JM, Staudt LM, Hudson J Jr, Boguski MS et al. 1999 The transcriptional program in the response of human fibroblasts to serum. Science 283 83-87.

de Jager T, T, Mueller-Botz S, Imam A, Muck J \& Neyses L 2001 Mechanisms of estrogen receptor action in the myocardium: rapid gene activation via the ERK1/2 pathway and serum response elements. Fournal of Biological Chemistry 276 27873-27880.

Katoh M 2003 Expression and regulation of WNT1 in human cancer: up-regulation of WNT1 by beta-estradiol in MCF-7 cells. International Fournal of Oncology 22 209-212.

Kelly MJ \& Levin ER 2001 Rapid actions of plasma membrane estrogen receptors. Trends in Endocrinology and Metabolism 12 152-156.

Kudoh M, Knee DA, Takayama S \& Reed JC 2002 Bagl proteins regulate growth and survival of ZR-75-71 human breast cancer cells. Cancer Research 62 1904-1909.

Kyprianou N, English HF, Davidson NE \& Isaacs JT 1991 Programmed cell death during regression of the MCF-7 human breast cancer following estrogen ablation. Cancer Research $\mathbf{5 1}$ $162-166$.

Lercher MJ, Urrutia AO \& Hurst LD 2002 Clustering of housekeeping genes provides a unified model of gene order in the human genome. Nature Genetics 31 180-183.

Liu HL, Golder-Novoselsky E, Seto MH, Webster L, McClary J \& Zajchowski DA 1998 The novel estrogen-responsive B-box protein (EBBP) gene is tamoxifen-regulated in cells expressing an estrogen receptor DNA-binding domain mutant. Molecular Endocrinology 12 1733-1748.

Lobenhofer EK, Bennett L, Cable PL, Li L, Bushel PR \& Afshari CA 2002 Regulation of DNA replication fork genes by 17 beta-estradiol. Molecular Endocrinology 16 1215-1229.

Moggs JG \& Orphanides G 2001 Estrogen receptors: orchestrators of pleiotropic cellular responses. EMBO Reports 2 775-781.

Monni O, Barlund M, Mousses S, Kononen J, Sauter G, Heiskanen M, Paavola P, Avela K, Chen Y, Bittner ML et al. 2001 Comprehensive copy number and gene expression profiling of the 17 q23 amplicon in human breast cancer. PNAS 98 5711-5716.

Murayama Y, Hammond GL \& Sugihara K 1999 The shbg gene and hormone dependence of breast cancer: a novel mechanism of hormone dependence of MCF-7 human breast cancer cells based upon SHBG. Breast Cancer 6 338-343.

Nye AC, Rajendran RR, Stenoien DL, Mancini MA, Katzenellenbogen BS \& Belmont AS 2002 Alteration of large-scale chromatin structure by estrogen receptor. Molecular and Cellular Biology 22 3437-3449.

Oliver B, Parisi M \& Clark D 2002 Gene expression neighborhoods. Fournal of Biology $14 \cdot 1-4 \cdot 3$.

Pawson T \& Saxton TM 1999 Signaling networks - do all roads lead to the same genes? Cell $\mathbf{9 7}$ 675-678.

Perillo B, Sasso A, Abbondanza C \& Palumbo G 2000 17 $\beta$-Estradiol inhibits apoptosis in MCF-7 cells, inducing bcl-2 expression via two estrogen-responsive elements present in the coding sequence. Molecular and Cellular Biology 20 2890-2901. 
Perissi V, Menini N, Cottone E, Capello D, Sacco M, Montaldo F \& De Bortoli M 2000 AP-2 transcription factors in the regulation of ERBB2 gene transcription by oestrogen. Oncogene 19 280-288.

Perou CM, Sorlie T, Eisen MB, van de Rijn M, Jeffrey SS, Rees CA, Pollack JR, Ross DT, Johnsen H, Akslen LA et al. 2000 Molecular portraits of human breast tumours. Nature 406 747-752.

Qin C, Nguyen T, Stewart J, Samudio I, Burghardt R \& Safe S 2002 Estrogen up-regulation of p53 gene expression in MCF-7 breast cancer cells is mediated by calmodulin kinase IV-dependent activation of a nuclear factor kappaB/CGAAT-binding transcription factor-1 complex. Molecular Endocrinology 16 1793-1809.

Reik W \& Walter J 2001 Genomic imprinting: parental influence on the genome. Nature Reviews. Genetics 2 21-32.

Schena M, Shalon D, Davis RW \& Brown PO 1995 Quantitative monitoring of gene expression patterns with a complementary DNA microarray. Science 270 467-470.

Schofield PN, Joyce JA, Lam WK, Grandjean V, Ferguson-Smith A, Reik W \& Maher ER 2001 Genomic imprinting and cancer; new paradigms in the genetics of neoplasia. Toxicology Letters 120 151-160.

Seth P, Krop I, Porter D \& Polyak K 2002 Novel estrogen and tamoxifen induced genes identified by SAGE (Serial Analysis of Gene Expression). Oncogene 21 836-843.

Sorbello V, Fuso L, Sfiligoi C, Scafoglio C, Ponzone R, Biglia N, Weisz A, Sismondi P \& De Bortoli M 2003 Quantitative real-time RT-PCR analysis of eight novel estrogen-regulated genes in breast cancer. International Fournal of Biological Markers 18 123-129.

Soulez M \& Parker MG 2001 Identification of novel oestrogen receptor target genes in human ZR75-1 breast cancer cells by expression profiling. Fournal of Molecular 27 259-274.

Spellman PT \& Rubin GM 2002 Evidence for large domains of similarly expressed genes in the Drosophila genome. Fournal of Biology 1 5.1-5.8.
Tamayo P, Slonim D, Mesirov J, Zhu Q Kitareewan S, Dmitrovsky E, Lander ES \& Golub TR 1999 Interpreting patterns of gene expression with self-organizing maps: methods and application to hematopoietic differentiation. PNAS 96 2907-2912.

Tusher VG, Tibshirani R \& Chu R 2001 Significance analysis of microarrays applied to the ionizing radiation response. PNAS 98 5116-5121.

Weisz A, Basile W, Scafoglio C, Natoli G, Altucci L, Bresciani F, Facchiano A, Sismondi P, Cicatiello L \& De Bortoli M 2003 Molecular identification of ERalpha-positive breast cancer cells by the expression profile of an intrinsic set of hormone regulated genes. Fournal of Cellular Physiology (In Press).

Whitfield ML, Sherlock G, Saldanha AJ, Murray JI, Ball CA, Alexander KE, Matese JC, Perou CM, Hurt MM, Brown PO et al. 2002 Identification of genes periodically expressed in the human cell cycle and their expression in tumors. Molecular Biology of the Cell 13 1977-2000.

Xu CF, Chambers JA \& Solomon E 1997 Complex regulation of the BRCAl gene. Fournal of Biological Chemistry 272 20994-20997.

Yamada M, Yoshida M \& Hashinaka K 1993 Identification of transcriptional cis-elements in introns 7 and 9 of the myeloperoxidase gene. Journal of Biological Chemistry $\mathbf{2 6 8}$ 13479-13485.

Yue H, Eastman PS, Wang BB, Minor J, Doctolero MH, Nuttall RL, Stack R, Becker JW, Montgomery JR, Vainer M et al. 2001 An evaluation of the performance of cDNA microarrays for detecting changes in global mRNA expression. Nucleic Acids Research $29 \mathrm{E} 41 \cdot 1$.

Received in final form 20 November 2003 Accepted 20 February 2004 